\title{
Voisin-Borcea manifolds and heterotic orbifold models
}

\section{W. Buchmuller, ${ }^{a}$ J. Louis, ${ }^{b, c}$ J. Schmidt ${ }^{a}$ and R. Valandro ${ }^{b}$}

${ }^{a}$ Deutsches Elektronen-Synchrotron DESY, Notkestrasse 85, 22607 Hamburg, Germany

${ }^{b}$ II Institute for Theoretical Physics, Hamburg University, Luruper Chaussee 149, 22761 Hamburg, Germany

${ }^{c}$ Zentrum für Mathematische Physik, Hamburg University, Bundesstrasse 55, 20146 Hamburg, Germany

E-mail: buchmuwi@mail.desy.de, jan.louis@desy.de, schmijon@mail.desy.de, roberto.valandro@desy.de

ABSTRACT: We study the relation between a heterotic $T^{6} / \mathbb{Z}_{6}$ orbifold model and a compactification on a smooth Voisin-Borcea Calabi-Yau three-fold with non-trivial line bundles. This orbifold can be seen as a $\mathbb{Z}_{2}$ quotient of $T^{4} / \mathbb{Z}_{3} \times T^{2}$. We consider a two-step resolution, whose intermediate step is $\left(K 3 \times T^{2}\right) / \mathbb{Z}_{2}$. This allows us to identify the massless twisted states which correspond to the geometric Kähler and complex structure moduli. We work out the match of the two models when non-zero expectation values are given to all twisted geometric moduli. We find that even though the orbifold gauge group contains an $\mathrm{SO}(10)$ factor, a possible GUT group, the subgroup after higgsing does not even include the standard model gauge group. Moreover, after higgsing, the massless spectrum is non-chiral under the surviving gauge group.

Keywords: Superstrings and Heterotic Strings, Superstring Vacua

ARXIV EPRINT: 1208.0704 


\section{Contents}

1 Introduction 1

2 Heterotic compactifications to $D=6 \quad 3$

2.1 Heterotic compactification on $T^{4} / \mathbb{Z}_{3}$

2.2 Heterotic compactification on K3 with line bundles $\quad 7$

3 From 6D to 4D compactifications $\quad 13$

$\begin{array}{lll}3.1 & \text { Heterotic compactification on } T^{6} / \mathbb{Z}_{6-I I} & 13\end{array}$

3.2 Heterotic compactification on Voisin-Borcea CY three-folds 18

$\begin{array}{ll}3.3 & \text { Fluxes on the Voisin-Borcea manifold }\end{array}$

$\begin{array}{lll}3.4 & \text { Geometric moduli vs twisted states } & 29\end{array}$

3.5 The spectrum on the smooth Calabi-Yau 31

4 Conclusions $\quad 34$

A Geometric details $\quad 36$

A.1 Non-symplectic involutions on K3 surfaces 36

A.2 Blowing up the $A_{2}$-singularities $\quad 37$

$\begin{array}{lll}\text { A.3 Involution on the resolution of } T^{4} / \mathbb{Z}_{3} & 38\end{array}$

B 4D orbifold spectrum after higgsing $\quad 39$

$\begin{array}{lll}\text { C } & 4 \mathrm{D} \text { spectrum in the smooth case } & 39\end{array}$

\section{Introduction}

An important corner of the space of string vacua is populated by string orbifold compactifications [1-3]. In recent years, a landscape of phenomenologically interesting heterotic orbifold models has been constructed, which contain gauge group and chiral matter of the supersymmetric standard model. A large class of these models is based on the $E_{8} \times E_{8}$ heterotic string compactified on the $T^{6} / \mathbb{Z}_{6-I I}$ orbifold [4-9]. Employing worldsheet conformal field theory techniques the spectrum and some couplings in the effective low energy action have been computed.

On the other hand, these models also have 'unwanted' features such as a large number of massless states which form real representations with respect to the standard model gauge group but are chiral with respect to the full orbifold gauge group. To obtain realistic models one has to move away from the orbifold by higgsing part of the gauge group, which also generates masses for the vector-like part of the spectrum. In practice, this is 
rather cumbersome and the obtained results suffer from the fact that the exact form of the superpotential is unknown.

The appearance of vector-like massless fields might be an artifact of the construction at the orbifold point where generically additional symmetries and additional massless states appear. Therefore it is of interest to move away from the orbifold locus and construct the theories at generic points in their moduli space. Geometrically, this corresponds to blowing up the orbifold singularities and identifying smooth Calabi-Yau compactification manifolds. In this way, one may hope to relate the massless vector-like fields to geometric moduli and obtain a better understanding of their mass generation and the stabilization of the geometry.

The relation between heterotic orbifold models and their smooth counterparts has been actively investigated in recent years [10-16]. First it was studied from a local point of view: The orbifold singularity was blown up using toric methods [17] and then the match of the spectrum with the corresponding smooth compactification was established. Subsequently this was generalized to globally defined, compact orbifold backgrounds by gluing together patches related to different singularities to a Calabi-Yau three-fold with a gauge bundle [14]. Starting from standard model-like orbifold models on $T^{6} / \mathbb{Z}_{6-I I}$, it was found that the hypercharge $\mathrm{U}(1)$ gauge boson always becomes massive in the blow up process. In [15], this problem could be avoided by considering the orbifold $T^{6} /\left(\mathbb{Z}_{2}\right)^{3}$ where one of the $\mathbb{Z}_{2}$ factor acts freely on $T^{6}$. Recently, also Gauged Linear Sigma Models have been used to construct resolutions of toroidal orbifolds [18-20].

In this paper we study a specific $T^{6} / \mathbb{Z}_{6-I I}$ orbifold model which was previously analyzed from the perspective of grand unification in six dimensions [21, 22]. The orbifold under consideration can be viewed as the space $\left(T^{4} / \mathbb{Z}_{3} \times T^{2}\right) / \mathbb{Z}_{2}$, with an intermediate six-dimensional theory corresponding to the compactification on $T^{4} / \mathbb{Z}_{3}$. For an anisotropic compactification, with the size of $T^{4}$ small compared to the size of $T^{2}$, the latter can be related to the scale of grand unification. The four-dimensional theory is then obtained by further compactifying on $T^{2} / \mathbb{Z}_{2}$. For an example with non-zero Wilson lines it was shown that this two step procedure leads to the same spectrum as the direct compactification on $T^{6} / \mathbb{Z}_{6-I I}[21]$. To simplify the analysis we turn off all Wilson lines in the present paper.

Our goal is to identify a smooth manifold corresponding to the $T^{6} / \mathbb{Z}_{6-I I}$ orbifold and to determine its properties. In order to do so we again employ a two-step analysis. First we resolve $T^{4} / \mathbb{Z}_{3}$ to a smooth $\mathrm{K} 3$ surface and use the fact that the match of the spectra has already been established in [10]. In a second step we compactify on $T^{2}$ and divide out the $\mathbb{Z}_{2}$ action. This gives the singular space $\left(K 3 \times T^{2}\right) / \mathbb{Z}_{2}$ which can be resolved to obtain a smooth Calabi-Yau three-fold termed Voisin-Borcea manifold [23, 24].

In the orbifold under consideration the original $E_{8} \times E_{8}$ gauge group is broken by a specific gauge twist. In the smooth compactification this corresponds to the presence of a non-trivial gauge bundle. From the structure of the gauge bundle one can infer which twisted states correspond to geometric moduli and furthermore see that the transition from the orbifold to the smooth manifold is equivalent to a motion in the field space of twisted states. If these states are charged with respect to the gauge group at the orbifold point, spontaneous symmetry breaking occurs leaving a smaller gauge group in the smooth 
compactification. This allows us to identify which massless twisted states correspond to geometric Kähler moduli. This procedure differs from [14], where some Kähler moduli were identified with massive twisted states. Using properties of the Voisin-Borcea manifold we are also able to determine which twisted states of the orbifold correspond to geometric complex structure moduli.

We find that the vacuum expectation values (VEVs) needed to blow up the orbifold completely, break the orbifold gauge group to a small subgroup. In fact, to go to a smooth point in moduli space, all massless twisted states which correspond to geometric moduli have to acquire non-zero VEVs. Giving VEVs to all of these states breaks the orbifold gauge group, reducing consistently its rank. It turns out that the resulting light spectrum is non-chiral with respect to the unbroken gauge group. This perfectly matches with the results obtained for smooth compactifications.

The paper is organized as follows. In section 2 we review the correspondence between compactifications on the $T^{4} / \mathbb{Z}_{3}$ orbifold and a specific $K 3$ background with a line bundle. In this example we describe the techniques involved in matching the two backgrounds. In section 3 we turn to the four-dimensional backgrounds and use similar techniques to show the correspondence between orbifold and smooth compactifications. We first describe how to resolve the singular space $Y_{s}=\left(K 3 \times T^{2}\right) / \mathbb{Z}_{2}$ to a smooth Voisin-Borcea Calabi-Yau three-fold. After the identification of the proper gauge bundle, we give the map between the geometric moduli and the corresponding twisted states. We show that the resulting gauge group is the same and that the non-Abelian massless spectrum also matches. The three appendices give further details on K3 surfaces and blow-up of singularities, and on the massless spectra in four dimensions, for orbifold and Calabi-Yau three-fold.

\section{Heterotic compactifications to $D=6$}

In this section we consider the $E_{8} \times E_{8}$ heterotic string compactified on a four dimensional internal space. Specifically, we first study the compactification on the orbifold $T^{4} / \mathbb{Z}_{3}$ with a particular gauge twist but without Wilson lines. Subsequently we analyze the compactification of 10D supergravity on the resolved K3 surface with an appropriate gauge background. These backgrounds and the corresponding matching was studied in [10] and here we briefly discuss their results.

\subsection{Heterotic compactification on $T^{4} / \mathbb{Z}_{3}$}

In absence of Wilson lines, a heterotic orbifold model is completely specified by the action of the orbifold group on the internal geometry and on the gauge bundle. Let us start with the geometry.

Geometry of $T^{4} / \mathbb{Z}_{3}$. The action of a $\mathbb{Z}_{N}$ orbifold group on a four dimensional torus $T^{4}$ is specified by the twist vector $v=\left(v_{1}, v_{2}\right)$, where $N \cdot v_{i} \in \mathbb{Z}$. The $v_{i=1,2}$ determine the transformation of the two complex coordinates $z_{i}=x_{i}+\tau_{i} y_{i}$ of $T^{4}$ :

$$
\theta: \quad z_{i} \mapsto e^{2 \pi i v_{i}} z_{i}
$$




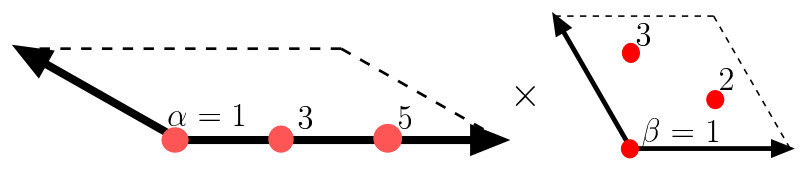

Figure 1. $\mathbb{Z}_{3}$ fixed points on $T^{4}=T^{2} \times T^{2}$. The 2-torus on the left is labelled by $z_{1}$, while the 2-torus on the right is labelled by $z_{2}$.

where $\tau_{i}$ denote the two complex structure parameters of $T^{4}$. To have a supersymmetric background the $v_{i}$ must satisfy $v_{1}+v_{2}=0 \bmod 1$. In the following we consider the specific case $N=3$ and $v=\left(-\frac{1}{3}, \frac{1}{3}\right)$. In order to have a $\mathbb{Z}_{3}$ symmetric $T^{4}, \tau_{1}$ and $\tau_{2}$ are fixed to generate the Lie algebra lattice of $G_{2} \times \mathrm{SU}(3)$.

The resulting geometry is a singular space with nine $\mathbb{C}^{2} / \mathbb{Z}_{3}$ orbifold singularities (see figure 1) at

$$
\left(z_{1}, z_{2}\right)=\left(z_{1}^{\alpha}, z_{2}^{\beta}\right) \quad \text { with } \quad \alpha=1,3,5 \text { and } \beta=1,2,3 .
$$

These are the points of $T^{4}$ left fixed by the $\mathbb{Z}_{3}$ action.

Furthermore, there are four two-cycles of $T^{4}$ surviving the orbifold projection. Expressed in terms of their Poicaré dual two-forms they are given by

$$
\begin{array}{ll}
\Pi_{1}=P D\left[3 d x_{2} \wedge d y_{2}\right], & \Theta_{1}=P D\left[-d x_{1} \wedge\left(2 d x_{2}+d y_{2}\right)-3 d y_{1} \wedge d x_{2}\right], \\
\Pi_{2}=P D\left[3 d x_{1} \wedge d y_{1}\right], & \Theta_{2}=P D\left[d x_{1} \wedge\left(d x_{2}-d y_{2}\right)-3 d y_{1} \wedge d y_{2}\right]
\end{array}
$$

where $P D[\cdot]$ indicates the Poincaré dual of the two-form in the bracket. $\Pi_{1}$ and $\Pi_{2}$ are the two $T^{2}$ spanned by $z_{1}$ and $z_{2}$. The other two two-cycles are combinations of the integral two-forms constructed from $d x_{1}, d y_{1}$ of the first $T^{2}$ and $d x_{2}, d y_{2}$ of the second $T^{2}$. One can check that all four two-cycles are invariant under $\mathbb{Z}_{3}$ which acts on the 1 -forms of $T^{4}$ as

$$
d x_{1} \mapsto-2 d x_{1}-3 d y_{1}, \quad d y_{1} \mapsto d x_{1}+d y_{1}, \quad d x_{2} \mapsto d y_{2}, \quad d y_{2} \mapsto-d x_{2}-d y_{2}
$$

The four two-cycles given in (2.3) have the intersection matrix:

$$
\left(\begin{array}{rrrr}
0 & 3 & & \\
3 & 0 & & \\
& & 2 & -1 \\
& & -1 & 2
\end{array}\right)
$$

where the first block contains $\Pi_{1}, \Pi_{2}$ while the second contains $\Theta_{1}, \Theta_{2}$. Note that the normalization of (2.5) is set by $\int_{T^{4}} d x_{1} d y_{1} d x_{2} d y_{2}=1$. Finally, the Kähler form $j$ and the holomorphic two-form $\Omega_{2}$ are given by

$$
j=t_{1} d x_{1} \wedge d y_{1}+t_{2} d x_{2} \wedge d y_{2}, \quad \quad \Omega_{2}=d z_{1} \wedge d z_{2}
$$

where $t_{1,2}$ are two Kähler parameters of $T^{4}$. 
Gauge twist and massless spectrum. The $\mathbb{Z}_{N}$ orbifold group also acts on the $E_{8} \times E_{8}$ gauge degrees of freedom by means of the twist vector

$$
V=\frac{1}{N}\left(n_{1}, \ldots, n_{8}\right)\left(n_{9}, \ldots, n_{16}\right),
$$

where the first (second) bracket acts on the first (second) $E_{8}$ factor and all $n_{a=1, \ldots, 16}$ are integers or half-integers. States with weight vector $w$ transform under the orbifold action with the phase $e^{2 \pi i V \cdot w}$. Modular invariance of the worldsheet partition function requires a relation between $V$ and the vector $v$ defined in the previous section

$$
N\left(\sum_{a=1}^{16} V_{a}^{2}-\sum_{i=1}^{2} v_{i}^{2}\right)=0 \bmod 2 .
$$

For the $T^{4} / \mathbb{Z}_{3}$ orbifold at hand, one can choose the gauge twist

$$
V=\left(0^{2}, \frac{2}{3}, 0^{5}\right)\left(\frac{2}{3}, 0^{7}\right)
$$

which breaks the gauge group from $E_{8} \times E_{8}$ to

$$
G=\mathrm{SO}(14) \times \mathrm{U}(1) \times \mathrm{SO}(14) \times \mathrm{U}(1) .
$$

The two U(1)'s have the following generators:

$$
T_{1}=\left(0^{2}, 2,0^{5}\right)\left(0^{8}\right), \quad T_{2}=\left(0^{8}\right)\left(2,0^{7}\right) .
$$

The massless spectrum can be organized in $6 \mathrm{D} \mathcal{N}=1$ supermultiplets. In the untwisted sector of the orbifold one finds hypermultiplets in the gauge representations

$$
(\mathbf{6 4}, \mathbf{1})_{1,0}+(\mathbf{1 4}, \mathbf{1})_{-2,0}+(\mathbf{1}, \mathbf{6 4})_{0,1}+(\mathbf{1}, \mathbf{1 4})_{0,-2}+2(\mathbf{1}, \mathbf{1})_{0,0},
$$

where the first (second) entry corresponds to the first (second) $\mathrm{SO}(14)$ factor and the subscripts denote the two $\mathrm{U}(1)$ charges. In addition one obtains the usual supergravity and dilaton multiplets, including the antisymmetric tensor $B$, and two hypermultiplets containing the 'radion' fields of the two tori. The twisted states are localized at the nine fixed points $\left(z_{1}^{\alpha}, z_{2}^{\beta}\right)$. At each fixed point there is a twisted spectrum with representations

$$
(\mathbf{1 4}, \mathbf{1})_{-\frac{2}{3}, \frac{4}{3}}+(\mathbf{1}, \mathbf{1 4})_{\frac{4}{3},-\frac{2}{3}}+2(\mathbf{1}, \mathbf{1})_{\frac{4}{3}, \frac{4}{3}} \cdot
$$

The $6 \mathrm{D}$ low energy theory around this orbifold vacuum contains a D-term potential for the scalars in the hypermultiplets. For canonically normalized scalars it reads

$$
V=\frac{1}{2} \sum_{i, A} D^{i, A} D^{i, A}, \quad \text { where } \quad D^{i, A}=\Phi_{m}^{\dagger} \sigma^{i} T_{m n}^{A} \Phi_{n}
$$

Here the $\Phi_{n}$ are doublets of complex scalars $\left(\phi_{n}, \tilde{\phi}_{n}\right), \sigma^{i}$ are the Pauli matrices and $T^{A}$ the generators of the gauge group. If the gauge group contains $\mathrm{U}(1)$ factors, then the corresponding generators are $T_{m n}^{A}=q_{n}^{A} \delta_{m n}$. 
As we will see shortly, the correspondence with the smooth $K 3$ compactification can be established by giving a VEV to a particular flat direction of the potential. Anticipating the result let us consider the singlets of $\mathrm{SO}(14) \times \mathrm{SO}(14)$ whose potential is solely determined by the $D$-terms of $\mathrm{U}(1) \times \mathrm{U}(1)$. From $(2.13)$ we see that at each singularity $\left(z_{1}^{\alpha}, z_{2}^{\beta}\right)$ there are two states $\Phi_{0}^{\alpha \beta}$ and $\Phi_{1}^{\alpha \beta}$ in the representation $(\mathbf{1}, \mathbf{1})_{4 / 3,4 / 3}$ which are charged under both U(1)s. This implies that flat directions of the potential are

$$
\left\langle\left(\phi_{0}^{\alpha \beta}, \tilde{\phi}_{0}^{\alpha \beta}\right)\right\rangle=\left(v^{\alpha \beta}, 0\right), \quad\left\langle\left(\phi_{1}^{\alpha \beta}, \tilde{\phi}_{1}^{\alpha \beta}\right)\right\rangle=\left(0, v^{\alpha \beta}\right),
$$

which break $\mathrm{U}(1) \times \mathrm{U}(1)$ to the diagonal $\mathrm{U}(1)$, leaving $\mathrm{SO}(14) \times \mathrm{SO}(14) \times \mathrm{U}(1)$ as the unbroken gauge group. The vector multiplet of the broken $\mathrm{U}(1)$ eats one hypermultiplet in the singlet representation of the unbroken group to give a massive vector multiplet. Note that there are also other flat directions which break $\mathrm{SO}(14) \times \mathrm{SO}(14) \times \mathrm{U}(1)$.

In order to facilitate the comparison with the smooth compactification in the next section, it is convenient to define new field variables $\varphi_{i}^{\alpha \beta}$ by the transformation

$$
\phi_{0}^{\alpha \beta} \equiv v^{\alpha \beta} e^{\varphi_{0}^{\alpha \beta}}, \quad \tilde{\phi}_{1}^{\alpha \beta} \equiv v^{\alpha \beta} e^{\varphi_{1}^{\alpha \beta}} .
$$

If the $\phi_{0}^{\alpha \beta}$ have charge $q$ and transform linearly under the $\mathrm{U}(1)$ gauge transformation $\phi_{0}^{\alpha \beta} \mapsto e^{i q \chi} \phi_{0}^{\alpha \beta}$, then the field $\varphi_{0}^{\alpha \beta}$ transforms non-linearly with a local shift

$$
\varphi_{0}^{\alpha \beta} \mapsto \varphi_{0}^{\alpha \beta}+i q \chi
$$

This gauged shift symmetry gives mass to the corresponding U(1) vector. The "axionic" fields $\varphi_{i}^{\alpha \beta}$ can also be used to redefine the other fields in the associated $(\alpha, \beta)$-twisted sector. This redefinition changes their charge with respect to the broken $\mathrm{U}(1)$. For example, multiplying the hypermultiplet $\Phi_{(1,14)}^{\alpha \beta}$ with charges $(4 / 3,-2 / 3)$ by $e^{-\varphi_{1}^{\alpha \beta}}$, one obtains 14 plets with charges $(0,-2)$. After analogous redefinition and including the untwisted states, one obtains the spectrum:

$$
(\mathbf{6 4}, \mathbf{1})_{1,0}+10(\mathbf{1 4}, \mathbf{1})_{-2,0}+(\mathbf{1}, \mathbf{6 4})_{0,1}+10(\mathbf{1}, \mathbf{1 4})_{0,-2}+20(\mathbf{1}, \mathbf{1})_{0,0} .
$$

Below the scale of symmetry breaking the unbroken gauge group only contains the diagonal U(1) with charges

$$
(\mathbf{6 4}, \mathbf{1})_{1}+(\mathbf{1}, \mathbf{6 4})_{-1}+10(\mathbf{1 4}, \mathbf{1})_{-2}+10(\mathbf{1}, \mathbf{1 4})_{+2}+19(\mathbf{1}, \mathbf{1})_{0},
$$

where furthermore one singlet was removed by the Higgs mechanism.

In addition to the flat direction considered so far there are other flat directions which break the orbifold gauge group to smaller subgroups. The following example is useful in order to understand the matching between orbifold compactifications with K3 compactifications. The vacuum is now characterized by expectation values of the non-Abelian singlet fields $\Phi_{0}^{\alpha \beta}$ and $\Phi_{1}^{\alpha \beta}$ as well as $\Phi_{(1,14)}^{\alpha \beta}$ :

$$
\begin{aligned}
\left.\left\langle\left(\phi_{0}^{\alpha \beta}, \tilde{\phi}_{0}^{\alpha \beta}\right)\right\rangle\right|_{\beta=1} & =(\sqrt{2} v, 0), & \left.\left\langle\left(\phi_{1}^{\alpha \beta}, \tilde{\phi}_{1}^{\alpha \beta}\right)\right\rangle\right|_{\beta=2,3} & =(0, v), \\
\left.\left\langle\left(\phi_{(1,14)}^{\alpha \beta}, \tilde{\phi}_{(1,14)}^{\alpha \beta}\right)\right\rangle\right|_{\beta=1} & =(\sqrt{2} \hat{v}, 0), & \left.\left\langle\left(\phi_{(1,14)}^{\alpha \beta}, \tilde{\phi}_{(1,14)}^{\alpha \beta}\right)\right\rangle\right|_{\beta=2,3} & =(0, \hat{v}) ;
\end{aligned}
$$


any component of the 14-plet may be chosen to have non-zero VEV. As we shall discuss in more detail in section 3.1, each component is characterized by a vector which is the sum of the shift vector $V$ and a root vector, e.g. $\tilde{p}=V+\left(0^{8}\right)\left(-1,1,0^{6}\right)$. One easily verifies that this configuation represents a flat direction. One of the $\mathrm{SO}(14)$ factors is now broken to $\mathrm{SO}(12)$, and the surviving gauge group reads

$$
\mathrm{SO}(14) \times \mathrm{SO}(12) \times \mathrm{U}(1)
$$

In the above example the same component of the 14-plet acquires a VEV at all fixed points. Choosing VEVs for different components on different fixed points one can achieve breaking to smaller subgroups.

The VEVs given in eqs. (2.15) and (2.20) correspond to directions in field space which lead away from the orbifold point into a smooth compactification. As we will see in the next section, the corresponding twisted states can be identified with the moduli controlling the sizes of collapsed cycles in the singularity. One expects that by giving large enough VEVs this procedure can be continued into a regime where the supergravity approximation of the heterotic string can be trusted.

\subsection{Heterotic compactification on K3 with line bundles}

In this section we briefly recall compactifications of $10 \mathrm{D}$ supergravity on smooth K3 manifolds in presence of a non-trivial gauge bundle [10, 25-27]. The goal is to find a set of backgrounds with appropriate flat direction that contain the orbifold $T^{4} / \mathbb{Z}_{3}$ as a limit in the K3 moduli space.

K3 moduli space. The metric of K3 is defined by the overall volume $\nu$ together with a three-dimensional subspace $\Sigma$ of the second cohomology group $H^{2}(K 3)$ (see [28] for a review). $H^{2}(K 3)$ is a 22 -dimensional vector space equipped with a natural scalar product of signature $(3,19)$ defined as

$$
(v \cdot w) \equiv \int_{K 3} v \wedge w, \quad \forall v, w \in H^{2}(K 3) .
$$

This product must be positive definite when restricted to $\Sigma$.

Taking three orthonormal vectors $\omega_{1}, \omega_{2}, \omega_{3}$ spanning the subspace $\Sigma$, one can define the Kähler form $j$ and the holomorphic two-form $\Omega_{2}:^{1}$

$$
j=\sqrt{2 \nu} \omega_{3}, \quad \Omega_{2}=\omega_{1}+i \omega_{2} .
$$

The motion in moduli space can be parametrized by the motion of the positive norm three-plane $\Sigma$ inside $H^{2}(K 3)$, i.e., by

$$
\mathcal{M}_{\Sigma}=\frac{\mathrm{SO}(3,19)}{\mathrm{SO}(3) \times \mathrm{SO}(19)} .
$$

\footnotetext{
${ }^{1}$ This definition is not unique: there is an $S^{2}$ of possible complex structures and associated Kähler forms with each of them defining the same metric.
} 


$$
T^{4} / \mathbb{Z}_{3}
$$

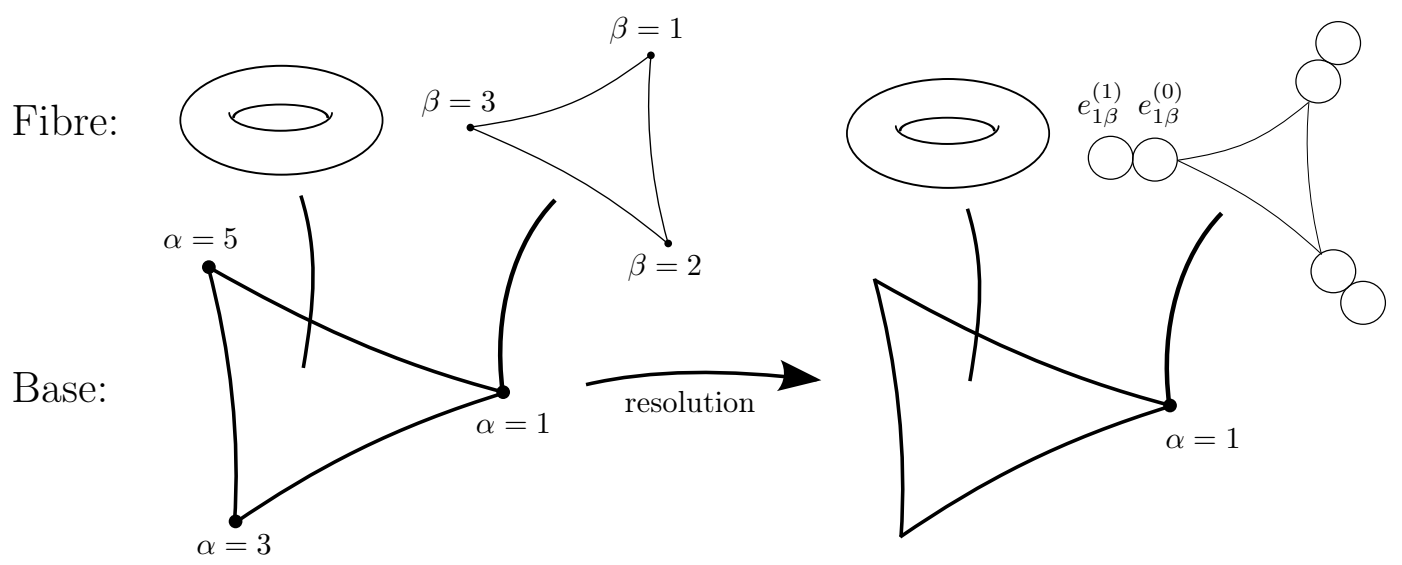

$K 3$

Figure 2. From $T^{4} / \mathbb{Z}_{3}$ to a smooth $K 3$.

$\mathcal{M}_{\Sigma}$ has 57 moduli, that together with the volume give the 58 geometric moduli of K3. Adding the 22 B-field moduli (i.e., the periods of the two-forms $B$ along the 22 harmonic two-form of K3), one obtains 80 moduli. These belong to 20 hypermultiplets of the $6 \mathrm{D}$ $\mathcal{N}=1$ supergravity coming from compactifying the heterotic theory on K3.

The position of $\Sigma$ in $H^{2}(K 3, \mathbb{Z})$ determines what cycles have zero size: If a two-cycle is orthogonal to $\Sigma$, it is shrunk to zero. If a set of two-cycles with self-intersection -2 (i.e., two-spheres) shrink, an orbifold singularity appears. These singularities are classified by ADE-groups that are subgroups of $E_{8} \times E_{8}$. K3 develops an ADE-singularity of type $G$ if the shrinking two-spheres have an intersection matrix equal to minus the Cartan matrix of the ADE group $G$.

From $\boldsymbol{T}^{4} / \mathbb{Z}_{\mathbf{3}}$ to a smooth K3. $T^{4} / \mathbb{Z}_{3}$ is a singular K3 that is elliptically fibered over the base $T^{2} / \mathbb{Z}_{3}$ (see figure 2). Over the singular points $z_{1}=z_{1}^{\alpha}$ of the base (spanned by $z_{1}$ ), the fiber (spanned by $z_{2}$ ) degenerates to $T^{2} / \mathbb{Z}_{3}$. $T^{4} / \mathbb{Z}_{3}$ has nine $A_{2}$ singularities (i.e., $\mathbb{C}^{2} / \mathbb{Z}_{3}$ singularities). This corresponds to the limit of a smooth $\mathrm{K} 3$ in which a set of cycles have shrunk to zero size. More precisely, each $A_{2}$ singularity is generated by two shrinking twocycles $e_{\alpha \beta}^{(0)}$ and $e_{\alpha \beta}^{(1)}$ where $\alpha \beta$ specifies the fixed point. Their intersection matrix is given by ${ }^{2}$

$$
\left(\begin{array}{cc}
-2 & 1 \\
1 & -2
\end{array}\right)
$$

with all intersections between cycles at different singularities vanishing.

There are two two-cycles at each of the nine singularities resulting in a total of 18 shrinking two-cycles, all of them orthogonal to the 3-plane $\Sigma$. Since $H^{2}(K 3)$ is 22 dimensional, $\Sigma$ still has the freedom to move in a four dimensional space with signature

\footnotetext{
${ }^{2}$ In the following we identify two-forms and two-cycles of K3, going from one to the other by Poincaré duality. On the space of two-cycles (two-forms) there is a natural inner product given by the intersection form or (2.22) respectively.
} 
$(3,1)$. Thus the number of geometrical moduli left is four which correspond to the overall volume and the parameters controlling the residual motion of $\Sigma$.

At the $T^{4} / \mathbb{Z}_{3}$ point of the moduli space, the lattice of cycles orthogonal to the holomorphic two-form $\Omega_{2}$ (the Picard lattice $P_{X}$ ) is (see [29])

$$
P_{X}=\mathcal{U} \oplus\left(-E_{6}\right) \oplus\left(-E_{6}\right) \oplus\left(-E_{6}\right) \quad \text { with } \quad \mathcal{U}=\left(\begin{array}{ll}
0 & 1 \\
1 & 0
\end{array}\right)
$$

and $\left(-E_{6}\right)$ denoting the Cartan matrix of $E_{6} \cdot{ }^{3}$ Therefore $P_{X}$ is a space of signature $(1,19)$. If $j$ stayed along the $\mathcal{U}$ part of the lattice, then the shrunken cycles would generate three $E_{6}$ singularities (remember that a cycle is shrunk to zero size if it is orthogonal to both $j$ and $\Omega_{2}$ ). To have nine $A_{2}$ singularities instead, $j$ must be orthogonal to the lattice ${ }^{4}$

$$
\Lambda^{\mathrm{shr}}=\left(-A_{2}\right)^{\oplus 9}
$$

In fact, $j$ is a combination of the two tori $\Pi_{1}$ and $\Pi_{2}$, with intersection matrix

$$
\left(\begin{array}{ll}
0 & 3 \\
3 & 0
\end{array}\right)
$$

that can be shown to be orthogonal to the $A_{2}$ cycles. They are linear combinations of the $\mathcal{U}$-block and the $E_{6}$ cycles.

So far we have listed only 20 independent two-cycles of the 22 cycles of $\mathrm{K} 3: \Pi_{1}, \Pi_{2}$, and the $18 A_{2}$ cycles. These span a sublattice of $P_{X}$ with the same rank. The orthogonal complement in $H_{2}(K 3, \mathbb{Z})$ is generated by the two cycles that span the $\Omega_{2}$-plane, i.e., the two cycles $\Theta_{1}$ and $\Theta_{2}$ of $T^{4}$ left invariant by $\mathbb{Z}_{3}$.

To go away from the orbifold point, $\Sigma$ has to move inside the space spanned by the cycles $e_{\alpha \beta}^{(0)}, e_{\alpha \beta}^{(1)}$, in such a way that they are no more orthogonal to it. In terms of the Kähler form and the holomorphic two-form, this means that they have non-zero components along the space spanned by $e_{\alpha \beta}^{(0)}, e_{\alpha \beta}^{(1)}$.

Fluxes on K3. In supergravity compactifications an additional constraint arises from the Bianchi identity of the $B$-field, which is given by ${ }^{5}$

$$
d H_{3}=\operatorname{tr} R \wedge R+\operatorname{tr} F \wedge F .
$$

Integrated over $K 3$ it yields

$$
\frac{1}{2} \int_{K 3} d H_{3}=\chi(K 3)+\frac{1}{2} \int_{K 3} \operatorname{tr} F \wedge F=0
$$

\footnotetext{
${ }^{3}$ We see the appearance of the Cartan matrix of $E_{6}$ in figure 2: The $E_{6}$ cycles are the $\mathbb{P}^{1}$ located at, for example, $\alpha=1$ on the right side of the figure. We see that their intersection matrix is actually the same as the Cartan matrix of $E_{6}$.

${ }^{4}$ In order to have a $A_{2}$ singularity, the corresponding lattice must be primitively embedded in $P_{X}$ (a lattice embedding $\ell \hookrightarrow L$ is primitive if $L / \ell$ is torsion-free).

${ }^{5}$ We use Hermitian generators.
} 
where

$$
\chi(K 3)=\int_{K 3} \operatorname{tr} R \wedge R=24
$$

is the Euler characteristic of K3. Eq. (2.29) implies that there has to be a non-trivial background gauge bundle on K3 which can either be non-Abelian (an instanton background) or Abelian (a flux background). In the following we consider Abelian fluxes and review how they generate the orbifold twist in the orbifold limit [14]. The specific background we are interested in is the model IIIb studied in ref. [10].

The Yang-Mills equation of motion plus the Bianchi identity imply that the field strength $F$ is harmonic. Therefore we can expand the Abelian background field strength as

$$
F=f^{I} \otimes \eta_{I}, \quad I=1, \ldots, 22,
$$

where $\left\{\eta_{I}\right\}$ is a basis of $H^{2}(K 3, \mathbb{Z})$ and $f^{I}$ are constant flux parameters in the Cartan subalgebra of $E_{8} \times E_{8}$. In the following we only consider fluxes that match with the orbifold data in the orbifold limit.

As explained in [13], the flux is related to the twist vector $V$ defined in (2.7) and (2.9). $k V$ gives the non-trivial boundary conditions around the fixed locus of $\theta^{k}$ defined in (2.1). At the orbifold point, this is equivalent to making the boundary conditions trivial, but at the price of switching on a nonzero background gauge field $\mathcal{A}$, that has zero field strength over all the space except on the singular points. This is done by performing a non-single valued gauge transformation (see [30] for a simple example). The resulting $\mathcal{A}$ is given by

$$
k V \equiv \oint_{\gamma_{\theta k}} \mathcal{A}=\int_{D_{\theta^{k}}} F
$$

where $D_{\theta^{k}}$ is a disk containing the singularity, and ' $\equiv$ ' means 'modulo vectors belonging to the $E_{8} \times E_{8}$ root lattice'. In the resolved space, this integral can be written as the intersection of two two-cycles: the two-cycle which is Poincaré dual to the flux $F$, that has zero size in the orbifold limit, and a smooth cycle of K3 that in the orbifold limit contains the singularity. The flux related to the twist is then proportional to the exceptional two-cycles (the new finite size cycle after resolution of the orbifold singularity). In particular, the flux related to the twist (2.9) can be chosen as

$$
F=\frac{1}{3} f \otimes \omega_{F}
$$

where

$$
\begin{aligned}
& \omega_{F}=\sum_{\alpha=1,3,5} \sum_{\beta=1,2,3}\left(e_{\alpha \beta}^{(1)}-e_{\alpha \beta}^{(0)}\right), \\
& \frac{1}{3} f=V, \quad f=\left(0^{2}, 2,0^{5}\right)\left(2,0^{7}\right) .
\end{aligned}
$$

$\omega_{F}$ is an integral two-form, written in terms of the Poincaré dual two-cycles. $f$ labels an element of the Cartan subalgebra of $E_{8} \times E_{8}$, which breaks $E_{8}$ to $\mathrm{SO}(14) \times \mathrm{U}(1)$. It is written in terms of an orthonormal basis with the convention $\operatorname{tr}_{E_{8} \times E_{8}} f_{1} f_{2}=\left(f_{1} \cdot f_{2}\right)$. 
The flux satisfies the tadpole cancellation condition (2.29):

$$
-\frac{1}{2} \int_{K 3} \operatorname{tr} F \wedge F=-\frac{1}{2} V^{2} \int \omega_{F} \wedge \omega_{F}=-\frac{1}{2} \times 8 \times(-6)=24,
$$

where we used that the intersection matrix of the 18 shrinking cycles generating the nine $\mathbb{Z}_{3}$ singularities $\left(-A_{2}\right)^{\oplus 9}$.

We now compute the massless spectrum given by this choice of flux (see [25]). The number of hypermultiplets transforming in the representation $\mathbf{R}$, associate to the bundle $W$, is given by the index (Riemann-Roch-Hirzebruch theorem [31])

$$
\chi(W)_{K 3}=\int_{K 3} \operatorname{ch}(W) \operatorname{Td}(K 3)=2 r+\operatorname{ch}_{2}(W),
$$

where $\operatorname{ch}(W)=r+c_{1}(W)+\operatorname{ch}_{2}(W)+\ldots$ is the total Chern character of the bundle $W, r$ is its rank and $\operatorname{Td}(K 3)=1+\frac{1}{12} c_{2}(K 3)+\ldots$ the Todd class of the tangent bundle of K3. In our case the connection on the vector bundle is Abelian and the representation is characterized by the charge $q$. In particular the number of hypermultiplets in a representation of charge $q$ is

$$
n_{\mathbf{R}}=1+\left(-3-q^{2} \operatorname{ch}_{2}(L)\right)=1+3\left(q^{2}-1\right) .
$$

The flux background (2.34) breaks each $E_{8}$ to $\mathrm{SO}(14) \times \mathrm{U}(1)$ with the adjoint 248 representation of $E_{8}$ decomposing as

$$
248=91_{0}+1_{0}+14_{-2}+64_{1}+14_{2}+\overline{64}_{-1}
$$

where $\mathbf{9} \mathbf{1}_{0}$ is the adjoint representation of $\mathrm{SO}(14)$. Using (2.38) one computes the multiplicities of the charged hypermultiplets to be

$$
n_{(64,1)_{1,0}}=1, \quad n_{(1,64)_{0,1}}=1, \quad n_{(14,1)_{-2,0}}=10, \quad n_{(1,14)_{0,-2}}=10 .
$$

Comparing with (2.19) we see that we have a match between the charged massless spectrum in the orbifold and $K 3$ background.

In $[25,27]$ it was shown that an Abelian flux also gauges the shift symmetry of the $B$-field with respect to the "fluxed" U(1). Through the Stückelberg mechanism, the vector boson of this $\mathrm{U}(1)$ becomes massive by eating one of the 20 hypermultiplets parameterizing the K3 moduli space. For the case at hand, the flux is switched on only along one direction in the Cartan subalgebra of $E_{8} \times E_{8}$. Hence only one combination of the two U(1)s is massive and the surviving gauge group is

$$
\mathrm{SO}(14) \times \mathrm{SO}(14) \times \mathrm{U}(1)
$$

which indeed coincides with the unbroken gauge group in the orbifold background after giving a VEV to the scalars corresponding to the sizes of the blown up cycles.

One can also find fluxes which break $E_{8} \times E_{8}$ to smaller subgroups. A set of fluxes generalizing the above example can be written as

$$
F=\frac{1}{3}\left(f_{2} \otimes \omega_{F}^{(2)}+f_{4} \otimes \omega_{F}^{(4)}\right),
$$


where

$$
\omega_{F}^{(2)}=\sum_{\alpha=1,3,5} \sum_{\beta=1,2,3} e_{\alpha \beta}^{(1)}, \quad \omega_{F}^{(4)}=\sum_{\alpha=1,3,5} \sum_{\beta=1,2,3} e_{\alpha \beta}^{(0)},
$$

with $f_{2}=\left(0^{2}, 2,0^{5}\right)\left(-1,1,0^{6}\right)$ and $f_{4}=\left(0^{2},-2,0^{5}\right)\left(-2,0^{7}\right)$. One easily verifies that this flux satisfies the tadpole cancellation condition. The unbroken group is now

$$
\mathrm{SO}(14) \times \mathrm{U}(1) \times \mathrm{SO}(12) \times \mathrm{U}(1)^{2} .
$$

This group coincides with the unbroken gauge group of the second vacuum considered in section 2.1, and one can check that also the massless spectra match.

It is instructive to write the flux in the form of the matching condition (2.32):

$$
\begin{array}{ll}
\frac{1}{3} f_{2}=V+p_{2}, & p_{2}=\left(0^{8}\right)\left(-1,1,0^{6}\right), \\
\frac{1}{3} f_{4}=2 V+p_{4}, & p_{4}=\left(0^{2},-2,0^{5}\right)\left(-2,0^{7}\right) .
\end{array}
$$

It is intriguing that the root vector $p_{2}$ is identical to the root vector of the twisted state which acquires a VEV in the corresponding orbifold vacuum. The root vector $p_{4}$ is parallel to the twist $V$. For comparison, in the first example discussed in this section, where both $\mathrm{SO}(14)$ factors remain unbroken, the root vector $p_{4}$ is the same whereas $p_{2}=0$. The connection between the root vectors appearing in the matching conditions (2.32) for fluxes and the root vectors of twisted states acquiring VEVs has been observed in many examples, although a deeper theoretical understanding needs further investigations.

The fluxes generate a D-term potential for the geometric moduli $[25,27]$ which vanishes for (cf. (2.23))

$$
F \wedge \omega_{i}=0
$$

i.e., when $F$ is orthogonal to the Kähler form $j$ and the holomorphic two-form $\Omega_{2}$. In our case, an example of a flat direction solving (2.47), with $F$ given by (2.33), reads

$$
\begin{aligned}
j & =j^{(0)}+\sum_{\alpha \beta} t^{\alpha \beta}\left(e_{\alpha \beta}^{(1)}+e_{\alpha \beta}^{(0)}\right), \\
\Omega_{2} & =\Omega_{2}^{(0)}+\sum_{\alpha \beta} z^{\alpha \beta}\left(e_{\alpha \beta}^{(1)}+e_{\alpha \beta}^{(0)}\right)+\left(\frac{2}{\nu} \sum_{\alpha \beta} t^{\alpha \beta} z^{\alpha \beta}\right) j^{(0)},
\end{aligned}
$$

where $t^{\alpha \beta}$ and $z^{\alpha \beta}$ are geometrical moduli fields and $j^{(0)}$ and $\Omega_{2}^{(0)}$ are the Kähler and holomorphic two-form at the singular point (i.e., when $t^{\alpha \beta}=z^{\alpha \beta}=0$ ). When $j$ and $\Omega_{2}$ have the forms defined above, the two-cycles $e_{\alpha \beta}^{(0)}$ and $e_{\alpha \beta}^{(1)}$ have the same size. ${ }^{6}$

We have switched on a flux that corresponds to the orbifold twist. This flux generates a D-term that leaves some flat directions. The corresponding orbifold twist also generates

\footnotetext{
${ }^{6}$ The size of a two-cycle $C$ of K3 is given by $\operatorname{vol}(C)=\sum_{a=1}^{3}\left|\int_{C} \omega_{a}\right|^{2}$, where $\left\{\omega_{a}\right\}$ is the orthonormal basis of $\Sigma$.
} 
a D-term leaving some flat directions. This allows us to relate the moduli on both sides: In particular we can relate the moduli controlling the size of $e_{\alpha \beta}^{(0)}$ and $e_{\alpha \beta}^{(1)}$ on the smooth side with the respective scalars in the hypermultiplets $\Phi_{0}^{\alpha \beta}$ and $\Phi_{1}^{\alpha \beta}$ in the twisted spectrum on the orbifold side. Giving VEVs to $\Phi_{i}^{\alpha \beta}$ corresponds to making the cycles $e_{\alpha \beta}^{(i)}$ have finite size. The D-term condition says that the size of $e_{\alpha \beta}^{(0)}$ must be equal to the size of $e_{\alpha \beta}^{(1)}$.

Hence, we see that we can go away from the orbifold point by giving a VEV to $\Phi_{i}^{\alpha \beta}$ along the flat direction. For large enough VEVs, we can match this flat direction with a flat direction in the geometric space of smooth compactifications of heterotic string theory, with a non trivial gauge background corresponding to the orbifold gauge twist.

\section{From $6 \mathrm{D}$ to $4 \mathrm{D}$ compactifications}

In this section we construct four-dimensional theories by appropriately compactifying the six-dimensional backgrounds discussed in the previous section. On the orbifold side, we consider the space $T^{6} / \mathbb{Z}_{6-I I}$ which can be seen as the two-step quotient of $T^{4} \times T^{2}$

$$
T^{6} / \mathbb{Z}_{6-I I}=\frac{\left(T^{4} / \mathbb{Z}_{3}\right) \times T^{2}}{\mathbb{Z}_{2}}
$$

Therefore we compactify the $T^{4} / \mathbb{Z}_{3}$ orbifold discussed in section 2.1 on $T^{2}$ and then mod out by a further $\mathbb{Z}_{2}$. This will introduce a new twisted sector coming from the $\mathbb{Z}_{2}$ quotient. For a specific choice of Wilson lines the equivalence of this approach with the direct compactification on the six-dimensional orbifold has been shown in ref. [21].

On the smooth side, we consider the singular space

$$
Y_{s}=\frac{\mathrm{K} 3 \times T^{2}}{\mathbb{Z}_{2}}
$$

and study the heterotic compactification on its resolution. As in $D=6$, we will show that the orbifold background can be viewed as a singular limit of an appropriate smooth compactification. Let us first discuss the orbifold theory.

\subsection{Heterotic compactification on $T^{6} / \mathbb{Z}_{6-I I}$}

To construct the orbifold $T^{6} / \mathbb{Z}_{6-I I}$ we consider the six torus $T^{6}=\mathbb{R}^{6} / 2 \pi \Lambda$, where $\Lambda$ is the $\mathbb{Z}_{6-I I}$ symmetric Lie algebra lattice of $G_{2} \times \mathrm{SU}(3) \times \mathrm{SO}(4)$. The $\mathbb{Z}_{6-I I}$ action $\theta$ on the three coordinates $z_{i=1,2,3}$ of the $T^{6}$ is given by

$$
\theta: z_{i} \mapsto e^{2 \pi v_{6}^{i}} z_{i} \quad \text { with } \quad v_{6}=\left(-\frac{1}{6},-\frac{1}{3}, \frac{1}{2}\right) .
$$

This orbifold has the following fixed loci $[4,6]$ (see figure 3):

- $\theta$ has 12 fixed points at $\left(z_{1}, z_{2}, z_{3}\right)=\left(z_{1}^{\alpha=1}, z_{2}^{\beta}, z_{3}^{\gamma}\right)$ with $\beta=1,2,3$ and $\gamma=1, \ldots, 4$,

- $\theta^{2}$ has 6 fixed planes at $\left(z_{1}, z_{2}\right)=\left(z_{1}^{\alpha}, z_{2}^{\beta}\right)$ with $\alpha=1,3,5$ and $\beta=1,2,3$, where $\alpha=3$ and $\alpha=5$ are identified after the orbifold projection, 

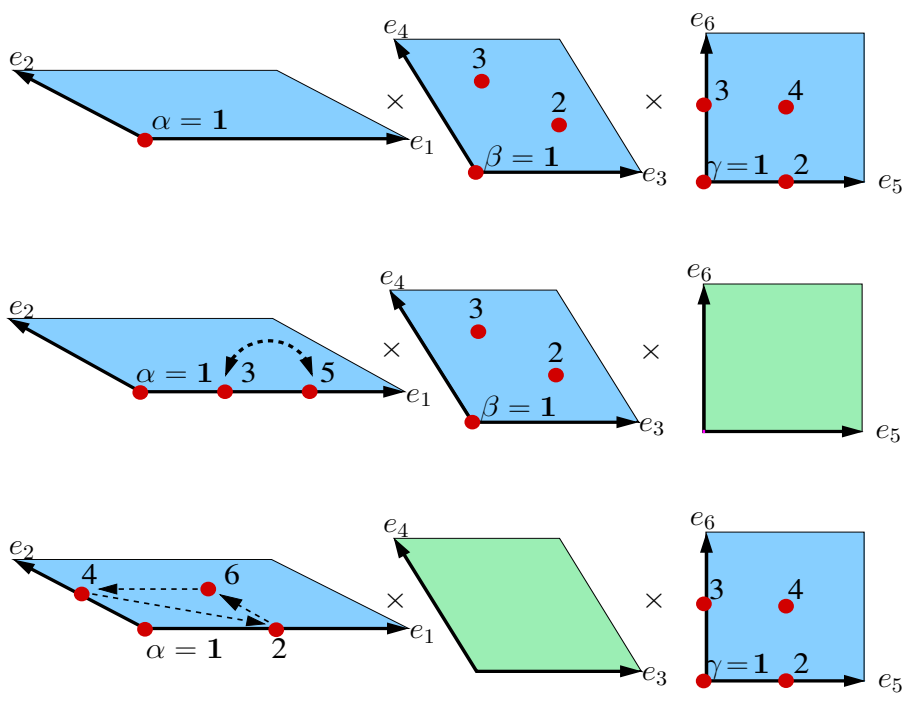

Figure 3. Fixed points and fixed planes of $T^{6} / \mathbb{Z}_{6-I I}$ orbifold for twists $\theta^{k}$. $\theta: 12$ fixed points (upper row); $\theta^{2}: 6$ fixed planes (middle row); $\theta^{3}: 8$ fixed planes (lower row) (from [14]).

- $\theta^{3}$ has 8 fixed planes at $\left(z_{1}, z_{2}\right)=\left(z_{1}^{\alpha}, z_{3}^{\gamma}\right)$ with $\alpha=2,4,6$ and $\gamma=1, \ldots, 4$, where $\alpha=2, \alpha=4$ and $\alpha=6$ are identified after the orbifold projection.

An important observation is that the orbifold twist $v_{6}$ is the sum of a $\mathbb{Z}_{3}$ and a $\mathbb{Z}_{2}$ twist vector. The first one acts only on $\left(z_{1}, z_{2}\right)$, while the second one acts only on $\left(z_{1}, z_{3}\right)$

$$
v_{6}=-v_{3}+v_{2}, \quad \text { where } \quad v_{3}=2 v_{6}, \quad v_{2}=3 v_{6} .
$$

This allows us to reconstruct the $T^{6} / \mathbb{Z}_{6-I I}$ orbifold in two steps by first acting with the $\mathbb{Z}_{3}$ projection and then with the $\mathbb{Z}_{2}$ projection as indicated in eq. (3.1).

The orbifold twist is embedded into the gauge group by the gauge twist

$$
V_{6}=\left(\frac{1}{2}, \frac{1}{2}, \frac{1}{3}, 0^{5}\right)\left(\frac{1}{3}, 0^{7}\right) \text {. }
$$

Also the gauge twist is a sum of a $\mathbb{Z}_{3}$ twist and a $\mathbb{Z}_{2}$ twist,

$$
V_{6}=-V_{3}+V_{2}, \quad \text { where } \quad V_{3}=2 V_{6}, \quad V_{2}=3 V_{6} .
$$

With this twist and vanishing Wilson lines, the unbroken gauge group is

$$
\mathrm{SU}(2)^{2} \times \mathrm{SO}(10) \times \mathrm{U}(1) \times[\mathrm{SO}(14) \times \mathrm{U}(1)],
$$

where the factor in brackets descends from the second $E_{8}$. The two U(1) generators are

$$
T_{1}=\left(0^{2}, 2,0^{5}\right)\left(0^{8}\right), \quad T_{2}=\left(0^{8}\right)\left(2,0^{7}\right) .
$$

One can check that only one linear combination of these two U(1)s is non-anomalous. The anomalous and non-anomalous $\mathrm{U}(1)$ factors are generated by

$$
T_{\text {non-an }}=3 T_{1}+T_{2} \quad T_{\text {anom }}=-T_{1}+3 T_{2} .
$$


Massless states are characterized by the twist number $k$ and by momentum vectors $q$ and $p$. They specify the Lorentz and gauge quantum numbers of the string states and are elements of the $\mathrm{SO}(8)$ weight lattice and the $E_{8} \times E_{8}$ root lattice. States of the heterotic string are given by a direct product of the right-moving and left-moving parts. A basis in the Hilbert space of the quantized string is obtained by acting with creation operators on the ground states of untwisted and twisted sectors $|q, p\rangle_{(k)}=\left|q+k v_{6}\right\rangle \otimes\left|p+k V_{6}\right\rangle, k=0 \ldots 4$. The vectors $\tilde{p}=p+V_{6}$ are called shifted momenta. The excited states obtained by acting with creation operators on the ground states are referred to as oscillator states.

Massless states of the untwisted and twisted sectors satisfy the mass equations

$$
\begin{aligned}
& \frac{1}{8} m_{\mathrm{L}}^{2}=\frac{1}{2} p^{2}-1+\widetilde{N}+\widetilde{N}^{*}=0, \\
& \frac{1}{8} m_{\mathrm{L}}^{2}=\frac{1}{2}\left(p+k V_{6}\right)^{2}-1+\delta c^{(k)}+w_{i}^{(k)} \widetilde{N}_{i}+\bar{w}_{i}^{(k)} \widetilde{N}_{i}^{*}=0,
\end{aligned}
$$

where

$$
\delta c^{(k)}=\frac{1}{2} \sum_{i} w_{i}^{(k)}\left(1-w_{i}^{(k)}\right)
$$

with $w_{i}^{(k)}=\left(k v_{N}\right)_{i} \bmod 1,0<w_{i}^{(k)} \leq 1$, and $\bar{w}_{i}^{(k)}=\left(-k v_{N}\right)_{i} \bmod 1,0<\bar{w}_{i}^{(k)} \leq 1$; $\widetilde{N}, \widetilde{N}^{*}, \widetilde{N}_{i}$ and $\widetilde{N}_{i}^{*}$ denote various oscillator numbers. In addition physical states have to satisfy projection conditions which relate the vectors $q$ and $p$.

As an example consider twisted matter fields of the $T_{1}$ sector. With $\delta c^{(1)}=11 / 36$ they satisfy the masslessness condition for the shifted momenta $\tilde{p}=p+V_{6}$,

$$
\left(p+V_{6}\right)^{2}=\frac{25}{18}
$$

A solution for $p+V_{6}$ is given by

$$
p+V_{6}=\left(0,0,-\frac{1}{6}, \text { odd }\left( \pm \frac{1}{2}\right)^{5}\right)\left(\frac{1}{3}, 0^{7}\right),
$$

where "odd $( \pm 1 / 2)^{5}$ " denotes all combinations containing an odd number of minus signs. These states form a 16-plet of $\mathrm{SO}(10)$.

A standard calculation (see e.g. $[32,33])$ yields the massless spectrum listed in table 1. The unwisted sector corresponds to $k=0$, the twisted sectors are labeled by $k=1,2,3,4$. $\alpha, \beta, \gamma$ denote which fixed point locus the twisted sectors live on (see figure 3). For example, for $\alpha=1, \beta=1, \gamma=1$, the corresponding twisted states are localized at the point $\left(z_{1}, z_{2}, z_{3}\right)=\left(z_{1}^{\alpha=1}, z_{2}^{\beta=1}, z_{3}^{\gamma=1}\right)$. For fixed planes, one of the indices is blank, meaning that the twisted states live on the whole corresponding plane. Some more explanations are needed for the index $\alpha$ : When it takes values ' $3+5$ ' (' $3-5$ '), this means that the physical states are even (odd) combinations of the $6 \mathrm{D}$ twisted states spanning the planes at $\alpha=3$ and $\alpha=5$. Analogously, states labeled by $\alpha=2+\nu^{k} 4+\nu^{2 k} 6\left(k=0,1,2, \nu=e^{-2 \pi i / 3}\right)$ are linear combinations with the phases $\left(1, \nu^{k}, \nu^{2 k}\right)$ of twisted states coming from the planes at $\alpha=2,4,6$. 


\begin{tabular}{|l|c|c|c|c|c|c|}
\hline Multiplet & $k$ & $\alpha$ & $\beta$ & $\gamma$ & $T_{1}$ & $T_{2}$ \\
\hline$(1,1,1 ; 64)$ & 0 & - & - & - & 0 & 1 \\
$(2,2,10 ; 1)$ & 0 & - & - & - & 0 & 0 \\
$(1,2,16 ; 1)$ & 0 & - & - & - & 1 & 0 \\
$(2,1,16 ; 1)$ & 0 & - & - & - & -1 & 0 \\
$(1,1,1 ; 14)$ & 0 & - & - & - & 0 & 2 \\
$(1,1,10 ; 1)$ & 0 & - & - & - & 2 & 0 \\
$(2,2,1 ; 1)$ & 0 & - & - & - & -2 & 0 \\
\hline$(1,1,16 ; 1)$ & 1 & 1 & $1,2,3$ & $1,2,3,4$ & $-1 / 3$ & $2 / 3$ \\
$(2,1,1 ; 1)$ & 1 & 1 & $1,2,3$ & $1,2,3,4$ & $2 / 3$ & $2 / 3$ \\
$(2,1,1 ; 1)$ & 1 & 1 & $1,2,3$ & $1,2,3,4$ & $2 / 3$ & $2 / 3$ \\
$(1,2,1 ; 1)$ & 1 & 1 & $1,2,3$ & $1,2,3,4$ & $-4 / 3$ & $2 / 3$ \\
\hline$(1,1,1 ; 1)$ & 2 & 1 & $1,2,3$ & - & $4 / 3$ & $4 / 3$ \\
$(2,2,1 ; 1)$ & 2 & 1 & $1,2,3$ & - & $-2 / 3$ & $4 / 3$ \\
\hline$(1,1,1 ; 1)$ & 2 & $3+5$ & $1,2,3$ & - & $4 / 3$ & $4 / 3$ \\
$(2,2,1 ; 1)$ & 2 & $3+5$ & $1,2,3$ & - & $-2 / 3$ & $4 / 3$ \\
\hline$(1,1,1 ; 1)$ & 2 & $3-5$ & $1,2,3$ & - & $4 / 3$ & $4 / 3$ \\
$(1,1,10 ; 1)$ & 2 & $3-5$ & $1,2,3$ & - & $-2 / 3$ & $4 / 3$ \\
$(1,1,1 ; 14)$ & 2 & $3-5$ & $1,2,3$ & - & $4 / 3$ & $-2 / 3$ \\
\hline$(1,1,1 ; 1)$ & 4 & 1 & $1,2,3$ & - & $-4 / 3$ & $-4 / 3$ \\
$(1,1,10 ; 1)$ & 4 & 1 & $1,2,3$ & - & $2 / 3$ & $-4 / 3$ \\
$(1,1,1 ; 14)$ & 4 & 1 & $1,2,3$ & - & $-4 / 3$ & $2 / 3$ \\
\hline$(1,1,1 ; 1)$ & 4 & $3+5$ & $1,2,3$ & - & $-4 / 3$ & $-4 / 3$ \\
$(1,1,10 ; 1)$ & 4 & $3+5$ & $1,2,3$ & - & $2 / 3$ & $-4 / 3$ \\
$(1,1,1 ; 14)$ & 4 & $3+5$ & $1,2,3$ & - & $-4 / 3$ & $2 / 3$ \\
\hline$(1,1,1 ; 1)$ & 4 & $3-5$ & $1,2,3$ & - & $-4 / 3$ & $-4 / 3$ \\
$(2,2,1 ; 1)$ & 4 & $3-5$ & $1,2,3$ & - & $2 / 3$ & $-4 / 3$ \\
\hline$(1,2,1 ; 1)$ & 3 & 1 & - & $1,2,3,4$ & 0 & 2 \\
\hline$(1,2,1 ; 1)$ & 3 & $2+4+6$ & - & $1,2,3,4$ & 0 & 2 \\
\hline$(1,2,1 ; 1)$ & 3 & $2+\nu 4+\nu^{2} 6$ & - & $1,2,3,4$ & 0 & -2 \\
\hline$(1,2,1 ; 14)$ & 3 & $2+\nu^{2} 4+\nu^{4} 6$ & - & $1,2,3,4$ & 0 & 0 \\
\hline
\end{tabular}

Table 1. Quantum numbers and localization of orbifold massless spectrum: representations w.r.t. $\mathrm{SU}(2)^{2} \times \mathrm{SO}(10) \times \mathrm{SO}(14)$ with $\mathrm{U}(1)$ charges $T_{1}$ and $T_{2}$. Untwisted states $(k=0)$ are not localized. Twisted states are localized in all planes for $k=1$; they are not localized in the $\mathrm{SO}(4)$ plane $(\gamma)$ for $k=2,4$ and in the $\mathrm{SU}(3)$ plane $(\beta)$ for $k=3$, respectively. In the $G_{2}$ plane $(\alpha)$ linear combinations of states occur for the considered $\mathbb{Z}_{6}$ twist. 
Flat directions on the orbifold side. In a 4D supersymmetric gauge theory with an anomalous $\mathrm{U}(1)$, vanishing of the D-terms requires

$$
\begin{aligned}
D^{a} & =\sum_{i} \phi_{i}^{\dagger} T^{a} \phi_{i}=0 \\
D_{\text {anom }} & =\sum_{i} q_{\text {anom }}^{i}\left|\phi_{i}\right|^{2}+\frac{g M_{P}^{2}}{192 \pi^{2}} \operatorname{tr}\left(T_{\text {anom }}\right)=0 .
\end{aligned}
$$

To satisfy the last equation one needs non-zero VEVs for fields that carry opposite charges with respect to the one of the Fayet-Iliopoulos term.

The D-term conditions can be satisfied if there exists a monomial $I\left(\phi_{i}\right)$ of fields that is invariant under the non-anomalous $\mathrm{U}(1)$ 's and charged with respect to the anomalous $\mathrm{U}(1)$, with sign opposite to that of $\operatorname{tr} T_{\text {anom }}$ (in our case $\operatorname{tr} T_{\text {anom }}=480$ ). The fields entering the monomial are those that get non-zero VEV [34-37],

$$
\left\langle\frac{\partial I}{\partial \phi_{i}}\right\rangle=c\left\langle\phi_{i}^{*}\right\rangle
$$

where $\langle\ldots\rangle$ denotes a VEV and $c$ is some constant. Consequently, for a monomial

$$
I\left(\phi_{i}\right)=\phi_{1}^{n_{1}} \ldots \phi_{l}^{n_{l}}
$$

the various VEVs satisfy the relation

$$
\frac{\left|\phi_{1}\right|}{\sqrt{n_{1}}}=\ldots=\frac{\left|\phi_{l}\right|}{\sqrt{n_{l}}}
$$

Such flat directions indeed exist for the considered orbifold. Since we want to realize a complete blow-up, we are interested in vacua with non-vanishing VEVs at the locations of all fixed points and all fixed planes, therefore involving at least 26 different fields. As we shall see, depending on the nature of the singularity, even more than one field acquires a VEV. Such configurations turned out to be difficult to find and were searched for by means of a special computer code.

An example involving 42 fields is listed in table 2, where the shifted momenta, i.e. the gauge quantum numbers of the massless fields with non-zero VEVs, are given. The corresponding monomial reads

$$
\begin{aligned}
I\left(\phi_{k ; \alpha}^{\beta \gamma}\right)= & \left(\prod_{\beta, \gamma} \phi_{1}^{\beta \gamma}\right)^{2}\left(\prod_{\beta}\left(\phi_{2 ; 1}^{\beta} \phi_{2 ;+}^{\beta}\right)^{4}\left(\phi_{2 ;-}^{\beta} \phi_{4 ; 1}^{\beta}\right)\left(\phi_{4 ;+}^{\beta} \phi_{4 ;-}^{\beta}\right)^{2}\right) \\
& \times\left(\prod_{\gamma}\left(\phi_{3 ; 1}^{\gamma} \phi_{3 ;+}^{\gamma}\right)\left(\phi_{3 ; \nu}^{\gamma}\right)^{9}\right),
\end{aligned}
$$

where $\phi_{k ; \alpha}^{\beta \gamma}$ denotes a scalar field with quantum numbers specified by the shifted momentum $\tilde{p}_{k ; \alpha}^{\beta \gamma}$, with $k, \alpha, \beta$ and $\gamma$ specifying twist and location, respectively. If the dependence on one localization index is trivial, this index is omitted. 


\begin{tabular}{|c|c|c|c|c|c|c|}
\hline Multiplet & $k$ & $\alpha$ & $\beta$ & $\gamma$ & & Shifted Momenta $\tilde{p}=\left(p+k V_{6}\right)$ \\
\hline \multirow{4}{*}{$(1,1,16 ; 1)_{-\frac{1}{3}, \frac{2}{3}}$} & 1 & 1 & $1,2,3$ & 1 & $\tilde{p}_{1}^{\beta 1}$ & $\left(0^{2},-\frac{1}{6},-\frac{1}{2},-\frac{1}{2},-\frac{1}{2},-\frac{1}{2},-\frac{1}{2}\right)\left(\frac{1}{3}, 0^{7}\right)$ \\
\hline & 1 & 1 & $1,2,3$ & 2 & $\tilde{p}_{1}^{\beta 2}$ & $\left(0^{2},-\frac{1}{6},+\frac{1}{2},+\frac{1}{2},-\frac{1}{2},-\frac{1}{2},-\frac{1}{2}\right)\left(\frac{1}{3}, 0^{7}\right)$ \\
\hline & 1 & 1 & $1,2,3$ & 3 & $\tilde{p}_{1}^{\beta 3}$ & $\left(0^{2},-\frac{1}{6},+\frac{1}{2},-\frac{1}{2},+\frac{1}{2},+\frac{1}{2},+\frac{1}{2}\right)\left(\frac{1}{3}, 0^{7}\right)$ \\
\hline & 1 & 1 & $1,2,3$ & 4 & $\tilde{p}_{1}^{\beta 4}$ & $\left(0^{2},-\frac{1}{6},-\frac{1}{2},+\frac{1}{2},+\frac{1}{2},+\frac{1}{2},+\frac{1}{2}\right)\left(\frac{1}{3}, 0^{7}\right)$ \\
\hline$(1,1,1 ; 1)_{\frac{4}{3}, \frac{4}{3}}$ & 2 & 1 & $1,2,3$ & - & $\tilde{p}_{2 ; 1}^{\beta}$ & $\left(0,0, \frac{2}{3}, 0,0,0,0,0\right)\left(\frac{2}{3}, 0,0^{6}\right)$ \\
\hline$(1,1,1 ; 1)_{\frac{4}{3}, \frac{4}{3}}$ & 2 & $3+5$ & $1,2,3$ & - & $\tilde{p}_{2 ;+}^{\beta}$ & $\left(0,0, \frac{2}{3}, 0,0,0,0,0\right)\left(\frac{2}{3}, 0,0^{6}\right)$ \\
\hline$(1,1,1 ; 14)_{\frac{4}{3},-\frac{2}{3}}$ & 2 & $3-5$ & $1,2,3$ & - & $\tilde{p}_{2 ;-}^{\beta}$ & $\left(0,0, \frac{2}{3}, 0,0,0,0,0\right)\left(-\frac{1}{3},+1,0^{6}\right)$ \\
\hline$(1,1,1 ; 14)_{-\frac{4}{3}, \frac{2}{3}}$ & 4 & 1 & $1,2,3$ & - & $\tilde{p}_{4 ; 1}^{\beta}$ & $\left(0,0,-\frac{2}{3}, 0,0,0,0,0\right)\left(\frac{1}{3},-1,0^{6}\right)$ \\
\hline$(1,1,1 ; 14)_{-\frac{4}{3}, \frac{2}{3}}$ & 4 & $3+5$ & $1,2,3$ & - & $\tilde{p}_{4 ;+}^{\beta}$ & $\left(0,0,-\frac{2}{3}, 0,0,0,0,0\right)\left(-\frac{2}{3}, 0,0^{6}\right)$ \\
\hline$(1,1,1 ; 1)_{-\frac{4}{3},-\frac{4}{3}}$ & 4 & $3-5$ & $1,2,3$ & - & $\tilde{p}_{4 ;-}^{\beta}$ & $\left(0,0,-\frac{2}{3}, 0,0,0,0,0\right)\left(-\frac{2}{3}, 0,0^{6}\right)$ \\
\hline$(1,2,1 ; 1)_{0,2}$ & 3 & 1 & - & $1, \ldots, 4$ & $\tilde{p}_{3 ; 1}^{\gamma}$ & $\left(+\frac{1}{2},-\frac{1}{2}, 0,0,0,0,0,0\right)\left(1,0,0^{6}\right)$ \\
\hline$(1,2,1 ; 1)_{0,2}$ & 3 & $2+4+6$ & - & $1, \ldots, 4$ & $\tilde{p}_{3 ;+}^{\gamma}$ & $\left(-\frac{1}{2},+\frac{1}{2}, 0,0,0,0,0,0\right)\left(1,0,0^{6}\right)$ \\
\hline \multirow[t]{2}{*}{$(1,2,1 ; 1)_{0,-2}$} & & $2+\nu 4+\nu^{2} 6$ & - & 1,2 & $\tilde{p}_{3 ; \nu}^{\gamma}$ & $\left(+\frac{1}{2},-\frac{1}{2}, 0,0,0,0,0,0\right)\left(-1,0,0^{6}\right)$ \\
\hline & 3 & $2+\nu 4+\nu^{2} 6$ & - & 3,4 & $\tilde{p}_{3 ; \nu}^{\gamma}$ & $\left(-\frac{1}{2},+\frac{1}{2}, 0,0,0,0,0,0\right)\left(-1,0,0^{6}\right)$ \\
\hline
\end{tabular}

Table 2. Orbifold blow-up: 42 twisted multiplets with non-zero VEVs along a D-flat direction.

Switching on these VEVs breaks the orbifold gauge group $\mathrm{SU}(2)^{2} \times \mathrm{SO}(10) \times \mathrm{U}(1) \times$ $\mathrm{SO}(14) \times \mathrm{U}(1)$ to the subgroup

$$
\mathrm{SU}(2) \times \mathrm{SU}(3) \times[\mathrm{SO}(12)],
$$

where the unbroken $\mathrm{SU}(2)$ is one of the two $\mathrm{SU}(2)$ factors. In this process five $\mathrm{U}(1)$ factors from the first $E_{8}$ and two from the second $E_{8}$ become massive by the Higgs mechanism. The massless spectrum given in table 1 can now be expressed in terms of representations of the unbroken gauge group (3.20), and it turns out to be non-chiral (see appendix B). Part of the spectrum will become massive due to superpotential couplings. Knowledge of these couplings is also needed to find out whether the considered D-flat direction contains points in field space where all F-terms vanish, which is required for a vacuum with unbroken supersymmetry.

We emphasize that the complexity of the flat direction (3.19) is due to the used matching conditions (2.32) and the constraints imposed by the Bianchi identities on fluxes as discussed below. Without these constraints, much simpler flat directions can be found, which leave the GUT group factor $\mathrm{SO}(10)$ in (3.20) unbroken.

\subsection{Heterotic compactification on Voisin-Borcea CY three-folds}

Let us now turn to the smooth side and try to identify a Calabi-Yau three-fold that has $T^{6} / \mathbb{Z}_{6-I I}$ as an orbifold locus in its moduli space. To construct this space, we view the 


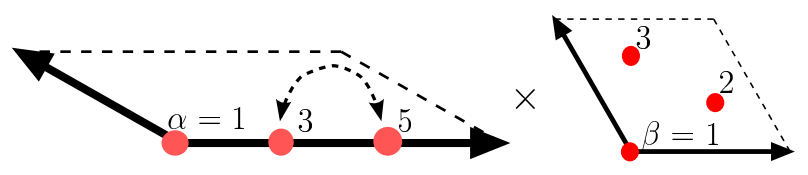

Figure $4 . \mathbb{Z}_{2}$ action on the $\mathbb{Z}_{3}$ fixed points of $T^{4}: \alpha=1$ is fixed, while $\alpha=3,5$ are exchanged.

orbifold as a two-step quotient as in eq. (3.1) and use the fact that $T^{4} / \mathbb{Z}_{3}$ can be resolved to a smooth K3, as we saw in section 2.2. This leaves us with the singular space

$$
Y_{s}=\frac{K 3 \times T^{2}}{\mathbb{Z}_{2}}
$$

where $\mathbb{Z}_{2}$ acts as a $z \rightarrow-z$ involution on $T^{2}$ and as a non-symplectic involution on K3 [3942]. The action on $T^{2}$ is independent of the resolution of the $T^{4} / \mathbb{Z}_{3}$ factor. Thus we are left to understand which involution on the smooth $\mathrm{K} 3$ extends the $\mathbb{Z}_{2}$ involution on $T^{4} / \mathbb{Z}_{3}$. An analogous analysis for the $T^{4} / \mathbb{Z}_{2}$ limit of $\mathrm{K} 3$ was done in [38], where the studied involution was the Enriques involution of $\mathrm{K} 3$ that extended a free action on $T^{4} / \mathbb{Z}_{2}$.

$\mathbb{Z}_{\mathbf{2}}$ involution. Let us first discuss the $\mathbb{Z}_{2}$ action on $T^{4} / \mathbb{Z}_{3}$. It acts on the complex coordinates of $T^{4}$ as

$$
\left(z_{1}, z_{2}\right) \mapsto\left(-z_{1}, z_{2}\right)
$$

From eq. (2.6) we see that under this action the holomorphic two-form $\Omega_{2}$ is odd while the Kähler-form $j$ is even,

$$
j \mapsto+j, \quad \Omega_{2} \mapsto-\Omega_{2} .
$$

Thus the $\mathbb{Z}_{2}$ acts non-trivially on $T^{4} / \mathbb{Z}_{3}$, and if we preserve this condition in the resolution we get a non-symplectic involution (see appendix A.1 for further details). The fixed loci of the $\mathbb{Z}_{2}$ action are:

- The set of points $\left\{\left(z_{1}, z_{2}\right) \mid z_{1}=z_{1}^{\alpha=1}\right\}$. This is a $T^{2} / \mathbb{Z}_{3}$ inside $T^{4} / \mathbb{Z}_{3}$, and it therefore has the topology of a two-sphere.

- The set of points $\left\{\left(z_{1}, z_{2}\right) \mid z_{1}=z_{1}^{\alpha=2} \vee z_{1}=z_{1}^{\alpha=4} \vee z_{1}=z_{1}^{\alpha=6}\right\}$. This set is connected and forms a $T^{2}$ inside $T^{4} / \mathbb{Z}_{3}$.

Let us now determine the action of $\mathbb{Z}_{2}$ on the singular points of $T^{4} / \mathbb{Z}_{3}$ and on its two-cycles. Looking at figure 4 , we see that inverting $z_{1}$, one position on the $G_{2}$ torus of the singular points (2.2) is fixed, while the other two are exchanged:

$$
\left(z_{1}^{\alpha=1}, z_{2}^{\beta}\right) \mapsto\left(z_{1}^{\alpha=1}, z_{2}^{\beta}\right), \quad\left(z_{1}^{\alpha=3}, z_{2}^{\beta}\right) \leftrightarrow\left(z_{1}^{\alpha=5}, z_{2}^{\beta}\right),
$$

while applying (3.22) on the four two-cycles defined in eq. (2.3) we obtain

$$
\Pi_{1} \mapsto+\Pi_{1}, \quad \Pi_{2} \mapsto+\Pi_{2}, \quad \Theta_{1} \mapsto-\Theta_{1}, \quad \Theta_{2} \mapsto-\Theta_{2} .
$$

We can resolve the orbifold singularities by moving the Kähler form inside the $e_{\alpha \beta^{-}}^{(i)}$ block (defined in section 2.2), while keeping $\Omega_{2}$ fixed. In this way the cycles $e_{\alpha \beta}^{(i)}$ have 


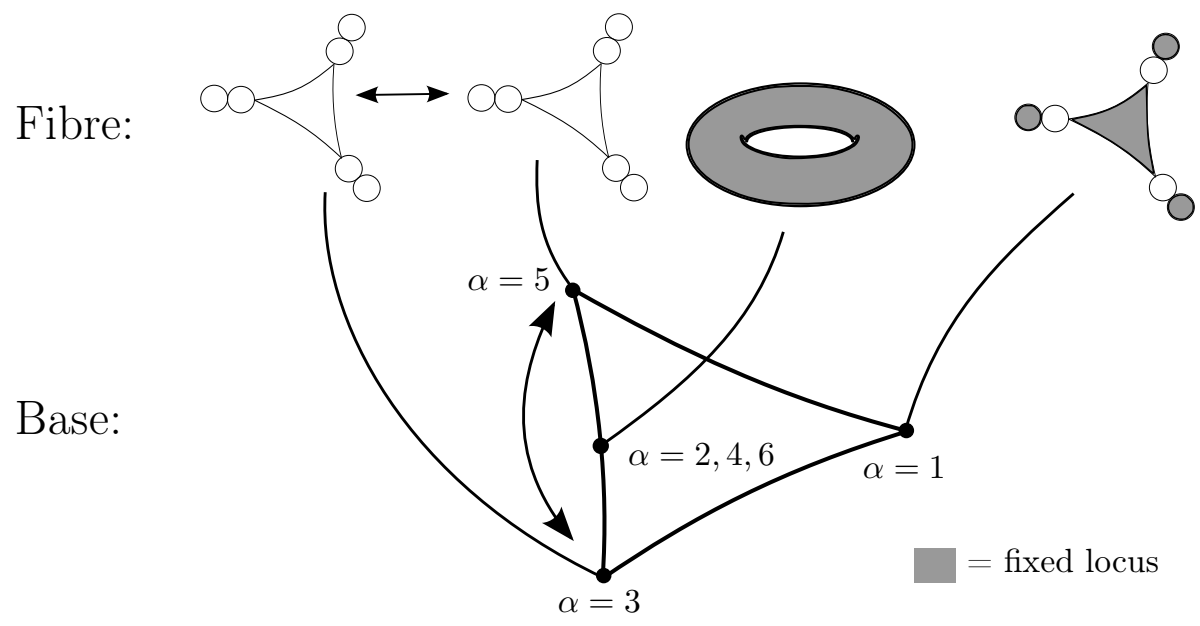

Figure 5. $\mathbb{Z}_{2}$ fixed locus on the smooth K3. For a detailed description, see appendix A.3.

non-zero size and belong to $H_{1,1}(K 3)$. The algebraic description of how to resolve any singular point by a Kähler deformation is given in appendix A.2. There it is also shown that from the transformation (3.24) of the singular points one can understand how the shrinking cycles $e_{\alpha \beta}^{(i)}$ transform under the $\mathbb{Z}_{2}$ involution (see figure 5),

$$
e_{1 \beta}^{(0)} \mapsto e_{1 \beta}^{(0)}, \quad e_{1 \beta}^{(1)} \mapsto e_{1 \beta}^{(1)}, \quad e_{3 \beta}^{(0)} \leftrightarrow e_{5 \beta}^{(0)}, \quad e_{3 \beta}^{(1)} \leftrightarrow e_{5 \beta}^{(1)} .
$$

The transformation of the remaining cycles is the same as in (3.25). Therefore, we have 14 independent $\mathbb{Z}_{2}$ even two-cycles:

$$
C_{\ell}^{K 3}: \quad \Pi_{1}, \Pi_{2}, e_{1 \beta}^{(0)}, e_{1 \beta}^{(1)}, e_{3 \beta}^{(0)}+e_{5 \beta}^{(0)}, e_{3 \beta}^{(1)}+e_{5 \beta}^{(1)} .
$$

Thus the rank of the even sublattice is equal to 14 . Furthermore, the fixed point locus $C_{\text {fix }}$ of $\mathbb{Z}_{2}$ acting on the smooth $\mathrm{K} 3$ is given by the following components:

- one $T^{2}$ at $z_{1}=z_{1}^{\alpha=2,4,6}$, that we call $C_{0}^{\mathrm{fix}}$, and one $\mathbb{P}^{1}$ at $z_{1}^{\alpha=1}$, that we call $C_{4}^{\mathrm{fix}}$ (these are the fixed loci of $T^{4} / \mathbb{Z}_{3}$ described above);

- three $\mathbb{P}^{1} \mathrm{~s}$ in the homology classes $e_{1 \beta}^{(1)}$ that we call $C_{\beta}^{\mathrm{fix}}$ (see appendix A.3).

With this information we are able to determine the non-symplectic involution corresponding to the orbifold $\mathbb{Z}_{2}$. As explained in appendix A.1, a non-symplectic involution of $\mathrm{K} 3$ is characterized by its action on the lattice of integral cycles. These involutions are classified in terms of the even sublattice $S_{+}(r, a, \delta)$, where $r=\operatorname{rk}\left(S_{+}\right)$[39-42]. The fixed locus is the disjoint union of $k \mathbb{P}^{1}$ 's and one curve of genus $g$ where $k=\frac{1}{2}(r-a)$ and $g=\frac{1}{2}(22-r-a)$ holds. The fixed locus found above determines $k$ and $g$ and thus $r$ and $a$ to be

$$
k=4, \quad g=1 \quad \Rightarrow \quad r=14, \quad a=6 .
$$


Note that $r=14$ was independently derived in eqs. (3.26), (3.27) from the transformations of the K3 cycles.

So far we have kept the holomorphic two-form $\Omega_{2}$ fixed at the $T^{4} / \mathbb{Z}_{3}$ point. We did this to make the cycles $e_{\alpha \beta}^{(i)}$ holomorphic and thus algebraically describable. On the other hand, the most generic symmetric point in the moduli space corresponds to $j$ being expanded along even cycles and $\Omega_{2}$ along odd ones. Their generic forms are then

$$
\begin{aligned}
j & =t_{1} \Pi_{1}+t_{2} \Pi_{2}+\sum_{i=0}^{1} \sum_{\beta=1}^{3}\left[t_{i}^{1 \beta} e_{1 \beta}^{(i)}+t_{i}^{3 \beta}\left(e_{3 \beta}^{(i)}+e_{5 \beta}^{(i)}\right)\right], \\
\Omega_{2} & =\zeta_{1}^{\Theta} \Theta_{1}+\zeta_{2}^{\Theta} \Theta_{2}+\sum_{i=0}^{1} \sum_{\beta=1}^{3} \zeta_{i}^{3 \beta}\left(e_{3 \beta}^{(i)}-e_{5 \beta}^{(i)}\right) .
\end{aligned}
$$

Altogether we have 14 Kähler moduli $t$ and 6 complex structure moduli $\zeta$. Note that $\zeta_{1}^{\Theta}$ and $\zeta_{2}^{\Theta}$ are fixed in terms of the $\zeta_{i}^{\alpha \beta}$ by requiring $\int \Omega_{2}^{2}=0$ and $\int \Omega_{2} \wedge \bar{\Omega}_{2}=1$.

Cycles in $\boldsymbol{Y}_{\boldsymbol{s}}$. Once we have identified the involution that acts on K3, the space $Y_{s}=$ $\left(K 3 \times T^{2}\right) / \mathbb{Z}_{2}$ in $(3.21)$ is determined. Since the $\mathbb{Z}_{2}$ involution has fixed points, $Y_{s}$ is singular. Before blowing up these singularities, let us describe the finite size cycles of $Y_{s}$ that are inherited from $K 3 \times T^{2}$. These cycles are products of K3 cycles with $T^{2}$ cycles that are not projected out by $\mathbb{Z}_{2}$. There are no 1 -cycles (and 5-cycles) surviving the $\mathbb{Z}_{2}$ projection. The surviving two-cycles are the $r=14$ even two-cycles of K3 and the even $T^{2}$, i.e. there are $r+1=15$. The surviving three-cycles are the product of the $22-r$ odd two-cycles of K3 and the 2 odd 1 -cycles of $T^{2}$, i.e. there are $2(22-r)=16$. The four-cycles are K3 itself (that we call $R_{3}$ in the following to match the notation of ref. [14]) and the products of even two-cycle $C_{\ell}^{K 3}$ of K3 (given in (3.27)) with $T^{2}$

$$
\left(C_{\ell}^{K 3} \times T^{2}\right) / \mathbb{Z}_{2}
$$

This is a singular four-dimensional submanifold if $C_{\ell}^{K 3}$ has fixed points under $\mathbb{Z}_{2}$.

From $Y_{s}$ to a smooth Voisin-Borcea manifold. So far we discussed the singular space $Y_{s}$ and thus the next step is to construct from it a smooth Calabi-Yau three-fold $Y$. We discuss this process keeping $r, a, k$ and $g$ generic. We use the fact that $Y_{s}$ can be viewed as an elliptic fibration over $K 3 / \mathbb{Z}_{2}$ where at a generic point of the $K 3 / \mathbb{Z}_{2}$ base the fiber is a $T^{2}[43] .{ }^{7}$ Over the $\mathbb{Z}_{2}$-fixed locus $C_{\text {fix }}$ inside K3, the fiber is the singular $T^{2} / \mathbb{Z}_{2}$ (see figure 6 on the left) with singularities being of type $A_{1}^{\oplus 4}$.

Now we can blow up the $A_{1}$ singularities to get the smooth $C Y$ three-fold $Y$. After the blow-up, the fiber over $C_{\text {fix }}$ is a collection of five (four independent) $\mathbb{P}^{1}$ 's intersecting like the extended Dynkin diagram of $\mathrm{SO}(8)$ [43] (see figure 6 on the right). The Hodge numbers of $Y$ are determined by $r$ and $a$ or alternatively by $k$ and $g$ to be [24, 43]

$$
\begin{aligned}
& h^{1,1}(Y)=5+3 r-2 a=r+1+4(k+1), \\
& h^{2,1}(Y)=65-3 r-2 a=1+(20-r)+4 g .
\end{aligned}
$$

These numbers can be understood in the following way:

\footnotetext{
${ }^{7} Y_{s}$ can also be understood as a K3 fibration over $T^{2} / \mathbb{Z}_{2}$.
} 


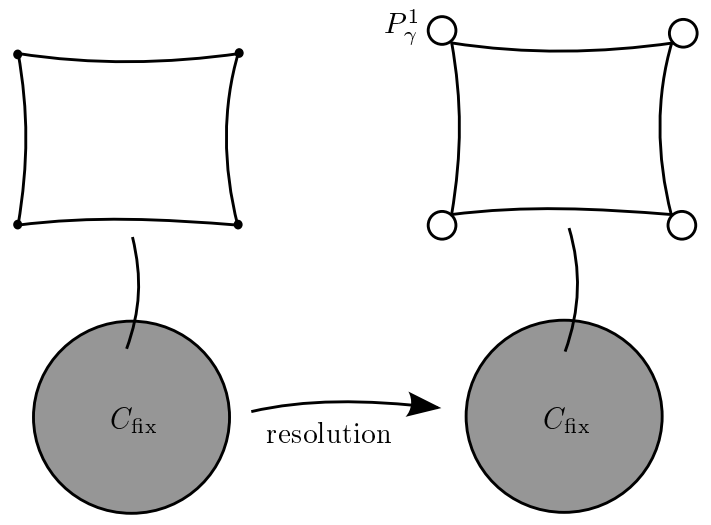

Figure 6. Resolution of the $\mathbb{C}^{2} / \mathbb{Z}_{2}$-singularities along the $\mathbb{Z}_{2}$-fixed locus of $\mathrm{K} 3$.

- $h^{1,1}$ counts the number of four-cycles. We have $(r+1)$ four-cycles determined above together with four four-cycles from the four blown-up $\mathbb{P}_{\gamma}^{1}(\gamma=1, \ldots, 4)$ fibered over the $\mathbb{Z}_{2}$-fixed curves $C_{\text {fix }}$. Since we have $(k+1)$ such curves, we expect $4(k+1)$ new four-cycles from the blow-up. This matches with the expression (3.31).

- $h^{2,1}$ is related to the number of three-cycles $b_{3}$ via $b_{3}=2\left(h^{2,1}+1\right)$. Above we determined the number of "bulk" three-cycles to be $2(22-r)$. After the blow-up, additional three-cycles arise as the product of the $2 g$ one-cycles in $C_{g}$ and the four independent two-cycles over $C_{g}$, i.e., altogether $8 g$ three-cycles. In total we then have $b_{3}=2\left(1+h^{2,1}\right)=2(22-r)+8 g$, which matches (3.32). We can alternatively understand $h^{2,1}$ by counting complex structure moduli of $Y$. There is one complex structure modulus of $T^{2}$ and $(20-r)$ possible complex structure deformations of $\Omega_{2}$. (Recall that $\Omega_{2}$ can be moved only in the space of odd cycles. ${ }^{8}$ ) Moreover there are $g$ complex structure deformations of the four $C_{g} \times \mathbb{P}_{\gamma}^{1}(\gamma=1, \ldots, 4)$.

Inserting (3.28) into (3.31) and (3.32) we obtain

$$
h^{1,1}(Y)=35, \quad h^{2,1}(Y)=11 .
$$

These are indeed the same Hodge numbers as obtained in the resolution of $T^{6} / \mathbb{Z}_{6-I I}$ in ref. [14]. In the following we will also see that the Voisin-Borcea manifold $Y$ is part of the possible triangulations given in [14]. We shall explicitly construct the $h^{1,1}(Y)=35$ four-cycles (divisors of $Y$ ) by following the two-step blow-up procedure outlined above, and we shall then connect them to the "sliding divisors" and the exceptional divisors obtained by toric resolution in [14].

Divisors in Voisin-Borcea CY three-fold. After the blow-up, the cycles (3.30) give rise to smooth four-cycles in $Y$. Let us briefly discuss their topology that depends on which even cycle $C_{\ell}^{K 3}$ is considered among the ones in (3.27):

\footnotetext{
${ }^{8}$ The number of moduli is determined by the motion of a positive norm two-plane in a space with signature $(2,20-r)$, and then is equal to the dimension of $\mathrm{SO}(2,20-r) / \mathrm{SO}(2) \times \mathrm{SO}(20-r)$.
} 
- $C_{\ell}^{K 3}=2 \Pi_{1}: C_{\ell}^{K 3}$ is given by two points on the $T^{2} / \mathbb{Z}_{3}$ base of the elliptically fibered $\mathrm{K} 3$ which are exchanged by $\mathbb{Z}_{2}$. We call the corresponding four-cycle $R_{1}$. It has the topology of $T^{2} \times T^{2}$.

- $C_{\ell}^{K 3}=\Pi_{2}$ : Before the blow-up, the corresponding four-cycle is given by $T^{4} / \mathbb{Z}_{2}$. We call its blow-up $R_{2}$. It is a K3 surface.

- $C_{\ell}^{K 3}=e_{1 \beta}^{(0)}$ : Before the blow-up the corresponding four-cycles are $\left(\mathbb{P}^{1} \times T^{2}\right) / \mathbb{Z}_{2}$. After the blow-up, they are called $E_{4,1 \beta}$ and have the topology of an Enriques surface.

- $C_{\ell}^{K 3}=e_{1 \beta}^{(1)}$ : The corresponding four-cycles, called $E_{2,1 \beta}$, have the topology of $\mathbb{P}^{1} \times \mathbb{P}^{1}$.

- $C_{\ell}^{K 3}=e_{3 \beta}^{(0)}+e_{5 \beta}^{(0)}, e_{3 \beta}^{(1)}+e_{5 \beta}^{(1)}$ : The corresponding four-cycles, called $E_{4,3 \beta}$ and $E_{2,3 \beta}$, have the topology of $\mathbb{P}^{1} \times T^{2}$.

To these divisors, we have to add the K3 divisor $R_{3}$ and the cycles that come from blowing up the $\mathbb{Z}_{2}$ singularities. They are given by the product

$$
C_{h}^{\mathrm{fix}} \times \mathbb{P}_{\gamma}^{1}
$$

where $\gamma=1, \ldots, 4$ labels the four $\mathbb{P}^{1}$ 's arising by the blow-up (see figure 6 ), and $C_{h}^{\text {fix }}$ are the $\mathrm{K} 3$ two-cycles fixed under $\mathbb{Z}_{2}$ shown in figure 5 . We recall that for $h=0, C_{h}^{\text {fix }}$ is the two-torus over $z_{1}=z_{1}^{\alpha=2,4,6}$, for $h=1,2,3 C_{h}^{\mathrm{fix}}$ are the fixed two-spheres located at $z_{1}=z_{1}^{\alpha=1}$ (in the homology class $e_{1 \beta}^{(1)}$ ), and for $h=4$ it is the singular fiber over $z_{1}=z_{1}^{\alpha=1}$ (see also appendix A for details). The topology of these cycles is easy to determine, as it follows from the topology of $C_{h}^{\text {fix }}$ and (3.34):

- When $h=0$, the corresponding four-cycles, that we call $E_{3,2 \gamma}$, have the topology of $T^{2} \times \mathbb{P}^{1}$.

- When $h=\beta=1,2,3$, the four-cycles, that we call $E_{1, \beta \gamma}$ are $\mathbb{P}^{1} \times \mathbb{P}^{1}$.

- When $h=4$, the four-cycles called $E_{3,1 \gamma}$ are $\mathbb{P}^{1} \times \mathbb{P}^{1}$.

We summarize the divisors of $Y$ in table 3 including the expected topology by specifying the Euler characteristic $\chi=h^{1,1}+2\left(1-2 h^{1,0}+h^{2,0}\right)$ and the holomorphic Euler characteristic $\chi_{0}=1-h^{1,0}+h^{2,0}$ (that are computed used the knowledge of the Hodge numbers of $T^{2}, \mathbb{P}^{1}, \mathrm{~K} 3$ and Enriques surface).

Above we have denoted the constructed divisors with the same names used in $[14,17]$ for the exceptional and inherited divisors in the one-step toric resolutions of $T^{6} / \mathbb{Z}_{6-I I}$. $R_{1}, R_{2}, R_{3}$ have been constructed in the same way and the exceptional divisors $E_{*, * *}$ have the same origin in the toric resolution [14, 17]:

- $E_{2,1 \beta}, E_{4,1 \beta}, E_{2,3 \beta}, E_{4,3 \beta}$ come from resolving the $\mathbb{Z}_{3}$ singularities (fixed planes spanning the third torus). In our construction they actually come from the K3 cycles obtained resolving the $\mathbb{Z}_{3}$ singularities; in particular, in both constructions $E_{2,3 \beta}, E_{4,3 \beta}$ are combinations of cycles in $\alpha=3$ and cycles in $\alpha=5$ like in our construction. 


\begin{tabular}{|c|c|c|c|c|}
\hline Divisor & K3 origin & Topology & $\chi$ & $\chi_{0}$ \\
\hline$R_{1}$ & $2 \Pi_{1}$ & $T^{2} \times T^{2}$ & 0 & 0 \\
$R_{2}$ & $\Pi_{2}$ & $K 3$ & 24 & 2 \\
$R_{3}$ & $\mathrm{~K} 3$ & $K 3$ & 24 & 2 \\
$E_{1, \beta \gamma}$ & $C_{\beta}^{\mathrm{fix}}$ & $\mathbb{P}^{1} \times \mathbb{P}^{1}$ & 4 & 1 \\
$E_{2,1 \beta}$ & $e_{1 \beta}^{(1)}$ & $\mathbb{P}^{1} \times \mathbb{P}^{1}$ & 4 & 1 \\
$E_{4,1 \beta}$ & $e_{1 \beta}^{(0)}$ & Enriques & 12 & 1 \\
$E_{2,3 \beta}$ & $e_{3 \beta}^{(1)}+e_{5 \beta}^{(1)}$ & $\mathbb{P}^{1} \times T^{2}$ & 0 & 0 \\
$E_{4,3 \beta}$ & $e_{3 \beta}^{(0)}+e_{5 \beta}^{(0)}$ & $\mathbb{P}^{1} \times T^{2}$ & 0 & 0 \\
$E_{3,1 \gamma}$ & $C_{4}^{\mathrm{fix}}$ & $\mathbb{P}^{1} \times \mathbb{P}^{1}$ & 4 & 1 \\
$E_{3,2 \gamma}$ & $C_{0}^{\mathrm{fix}}$ & $T^{2} \times \mathbb{P}^{1}$ & 0 & 0 \\
\hline
\end{tabular}

Table 3. The 35 ordinary and exceptional divisors of $Y$. In the second column we list the K3 two-cycles from which the divisors are constructed. The last three columns contain the topology of the divisors, Euler characteristic $\chi$ and holomorphic Euler characteristic $\chi_{0}$.

- $E_{3,1 \gamma}, E_{3,2 \gamma}$ come from resolving the $\mathbb{Z}_{2}$ singularities (fixed planes spanning the second torus). In our construction they come from finite size cycles of $T^{4} / \mathbb{Z}_{3}$ fixed by $\mathbb{Z}_{2}$.

- $E_{1, \beta \gamma}$ come from resolving the $\mathbb{Z}_{6}$ singularities (fixed points on $\alpha=1$ and spanned by $\beta, \gamma)$. In our construction they come from K3 cycles that were shrunk before the first blow up and then were at singular points of $T^{6} / \mathbb{Z}_{6-I I}$.

$\mathbb{C}^{3} / \mathbb{Z}_{3}$ can be torically resolved by five triangulation $[14,17]$. We now identify which of the five triangulations is the one corresponding to our construction. In our case, the cycles $E_{3}$ do not intersect the cycles $E_{1}$. In fact, they are just the product of $\mathbb{P}^{1}$ with $\mathbb{Z}_{2}$-fixed loci in K3 that do not intersect: $E_{1} \sim \mathbb{P}^{1} \times C_{\beta}^{\mathrm{fix}}$ and $E_{3} \sim \mathbb{P}^{1} \times C_{4}^{\mathrm{fix}}$ (see table 3 ); from figure 5 it is manifest that they do not intersect. There is only one triangulation that is compatible with this fact (the triangulation $(v)$ in $[14,17]$ at each fixed point, whose diagram is shown in figure 7). This allows us to use the result in $[14,17]$ for the intersection numbers of divisors and the characteristic classes of $Y .{ }^{9}$ The nonzero intersection numbers are:

$$
\begin{array}{rll}
R_{1} R_{2} R_{3}=1, & R_{2} E_{3,1 \gamma}^{2}=-2, & R_{2} E_{3,2 \gamma}^{2}=-6, \\
R_{3} E_{2,1 \beta} E_{4,1 \beta}=1, & R_{3} E_{2,1 \beta}^{2}=-2, & R_{3} E_{4,1 \beta}^{2}=-2, \\
R_{3} E_{2,3 \beta} E_{4,3 \beta}=2, & R_{3} E_{2,3 \beta}^{2}=-4, & R_{3} E_{4,3 \beta}^{2}=-4,
\end{array}
$$

where we use the abbreviation $D_{i} D_{j} D_{k}=\int_{Y} D_{i} \wedge D_{j} \wedge D_{k}$ and use the same symbol for the divisors and the Poincaré dual two-forms. The intersections (3.35) are triangulation

\footnotetext{
${ }^{9}$ These cycles do not form an integral basis, in the sense that the lattice $H_{4}(Y, \mathbb{Z})$ has also elements that are rational combination of them.
} 


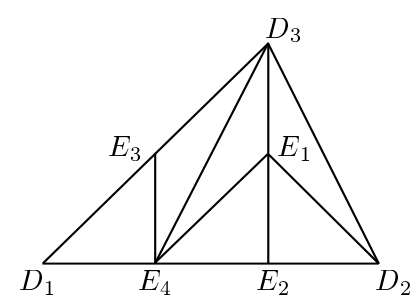

Figure 7. Triangulation $(v)$ of $\mathbb{C}^{3} / \mathbb{Z}_{6}$ corresponding to the Voisin-Borcea resolution. The vertices correspond to divisors. Two of them intersect each other if connected by a line (from $[14,17]$ ).

independent, i.e. they occur also for different toric resolution that are not related to our construction. ${ }^{10}$ The intersection numbers corresponding to our construction are:

$$
\begin{aligned}
& E_{1, \beta \gamma} E_{2,1 \beta} E_{4,1 \beta}=1, \quad E_{1, \beta \gamma} E_{2,1 \beta}^{2}=-2, \quad E_{1, \beta \gamma}^{2} E_{4,1 \beta}=-2, \\
& E_{2,1 \beta}^{2} E_{4,1 \beta}=-2, \quad E_{3,1 \gamma}^{2} E_{4,1 \beta}=-2, \quad E_{1, \beta \gamma}^{3}=8, \\
& E_{2,1 \beta}^{3}=8, \quad E_{3,1 \gamma}^{3}=8 .
\end{aligned}
$$

Note that the intersection between $E_{3}$ and $E_{1}$ is zero, as it must be.

Using toric geometry, one can also compute the characteristic classes of $Y$. Since $Y$ is Calabi-Yau we have trivially $c_{1}(Y)=0$. For $c_{2}(Y)$ one has $[14,17]$

$$
\begin{aligned}
c_{2}(Y)= & -\frac{25}{36} \sum_{\beta \gamma} E_{1, \beta \gamma}^{2}-\frac{5}{18} \sum_{\beta \gamma} E_{1, \beta \gamma} E_{2,1 \beta}-\frac{2}{9} \sum_{\beta \gamma} E_{1, \beta \gamma} E_{4,1 \beta}-\frac{1}{3} \sum_{\beta \gamma} E_{3,1 \gamma} E_{4,1 \beta} \\
& -\frac{7}{9} \sum_{\beta}\left(E_{2,1 \beta}^{2}+E_{4,1 \beta}^{2}+E_{2,3 \beta}^{2}+E_{4,3 \beta}^{2}\right)-\frac{4}{9} \sum_{\beta}\left(E_{2,1 \beta} E_{4,1 \beta}+E_{2,3 \beta} E_{4,3 \beta}\right) \\
& -\frac{3}{4} \sum_{\gamma}\left(E_{3,1 \gamma}^{2}+E_{3,2 \gamma}^{2}\right) .
\end{aligned}
$$

Once integrated on the divisor, $c_{2}(Y)$ gives the curvature contribution to the tadpole cancellation condition.

We can check that these data are consistent with the two-step construction: Using $c_{2}(Y)$ and the intersection numbers, one can derive the Euler characteristic $\chi$ and the holomorphic Euler characteristic $\chi_{0}$ of the divisors (see [17] for these definitions):

$$
\chi(S)=\int_{S} c_{2}(S)=c_{2}(Y) S+S^{3}, \quad \chi_{0}(S)=\frac{1}{12} \int_{S}\left(c_{1}(S)^{2}+c_{2}(S)\right)=\frac{1}{12}\left(S^{3}+\chi(S)\right),
$$

where we used $c_{1}(S)=-S$ and $c_{2}(S)=c_{2}(Y)+S^{2}$. The numbers that one obtains through these formulae are the same we have given in table 3 .

\footnotetext{
${ }^{10}$ We can understand these intersection numbers also in our construction. $Y$ can be seen as a K3 fibration over $T^{2} / \mathbb{Z}_{2}$. Let us consider $R_{3} E_{2,1 \beta} E_{4,1 \beta}=1$, as an example: $R_{3}$ is one point in the base $T^{2} / \mathbb{Z}_{2} ; E_{2,1 \beta}$ and $E_{4,1 \beta}$ intersect on a two-cycle that is one point in the K3 fiber and wraps the base. It is then easy to see that this two-cycle intersects $R_{3}$ once.
} 


\subsection{Fluxes on the Voisin-Borcea manifold}

In this section we determine the flux which is necessary to match the orbifold shift (3.5). We follow the same strategy as in the K3 analysis, i.e., the flux will be a linear combination of the exceptional two-forms with coefficients given by the gauge twist of the corresponding singularity. Going to the intermediate step of $\left(K 3 \times T^{2}\right) / \mathbb{Z}_{2}$, the flux reduces to a flux on $K 3$ plus a gauge twist on the singularity of $Y_{s}$. Following [14], the Ansatz for the flux is:

$$
\begin{aligned}
F= & \frac{1}{6} \sum_{\beta \gamma} f_{1}^{\beta \gamma} \otimes E_{1, \beta \gamma} \\
& +\frac{1}{3} \sum_{\beta}\left(f_{2}^{1 \beta} \otimes E_{2,1 \beta}+f_{4}^{1 \beta} \otimes E_{4,1 \beta}+f_{2}^{3 \beta} \otimes E_{2,3 \beta}+f_{4}^{3 \beta} \otimes E_{4,3 \beta}\right) \\
& +\frac{1}{2} \sum_{\gamma}\left(f_{3}^{1 \gamma} \otimes E_{3,1 \gamma}+f_{3}^{2 \gamma} \otimes E_{3,2 \gamma}\right),
\end{aligned}
$$

where the coefficients $f$ correspond to elements of the Cartan subalgebra. We now assume the matching conditions (2.32) [14]:

$$
\frac{1}{6} f_{1}^{\beta \gamma} \equiv V, \quad \frac{1}{3} f_{2}^{1 \beta} \equiv \frac{1}{3} f_{2}^{3 \beta} \equiv-\frac{1}{3} f_{4}^{1 \beta} \equiv-\frac{1}{3} f_{4}^{3 \beta} \equiv 2 V, \quad \frac{1}{2} f_{3}^{1 \gamma} \equiv \frac{1}{2} f_{3}^{2 \gamma} \equiv 3 V
$$

where $V$ is the orbifold shift given in eq. (3.5). These conditions also ensure the integrality of the flux.

If the flux coefficients $f$ were as in $(3.40)$ but with ' $=$ ' instead of ' $\equiv$ ', then the flux would break $E_{8} \times E_{8}$ to the orbifold gauge group (3.7). This would correspond to switching on only singlet VEVs in the non-Abelian factors. On the other hand, switching on fluxes that break the gauge group to some subgroup of (3.7) corresponds, on the orbifold side, to giving VEVs to twisted states that are charged under the non-Abelian part of (3.7). This correspondence has to work at any singularity: A flux along the exceptional cycle $E_{k}$ that breaks the gauge group further should correspond to a VEV of a twisted state in the sector $k$, that is in a representation of the non-Abelian part of the orbifold group and that leads to the same breaking. The precise prescription is given in $[14,15]$ : The shifted momentum $\tilde{p}_{k ;(i)}$ (cf. (3.13)) of the twisted state $\phi_{k ;(i)}$ taking a VEV, and localized at the singularity $(i)$, is identified with the coefficient of the flux along the exceptional divisor $E_{k, i}$.

In the following we analyze the consistency conditions one has to impose on the flux in a smooth compactification. In searching for fluxes that solve these conditions, we only allow for fluxes that can be related to the corresponding shifted momenta. In particular, they have to realize the same breaking of the gauge group.

Let us first analyze the consistency conditions imposed by the Bianchi identities. Since the field strength $H$ of the two-form field $B$ has to be globally defined one has

$$
\int_{S} d H=-\frac{1}{2} \int_{S} \operatorname{tr} F \wedge F-\int_{S} c_{2}(Y)=0, \quad \forall S
$$


where $S$ is any divisor of $Y$. We see that (3.41) relates fluxes and geometry. The divisors that give non-trivial conditions are $S=R_{2}, R_{3}, E_{1, \beta \gamma}, E_{2,1 \beta}, E_{3,1 \gamma}, E_{4,1 \beta}$ :

$$
\begin{aligned}
& R_{2}: \quad \sum_{\gamma}\left[\left\|\frac{1}{2} f_{3}^{1 \gamma}\right\|^{2}+3\left\|\frac{1}{2} f_{3}^{2 \gamma}\right\|^{2}\right]=24, \\
& R_{3}: \quad \sum_{\beta}\left[\left(\frac{1}{3} f_{2}^{1 \beta} ; \frac{1}{3} f_{4}^{1 \beta}\right)+2\left(\frac{1}{3} f_{2}^{3 \beta} ; \frac{1}{3} f_{4}^{3 \beta}\right)\right]=24 \text {, } \\
& E_{1, \beta \gamma}: \quad-4\left\|\frac{1}{6} f_{1}^{\beta \gamma}\right\|^{2}+\left\|\frac{1}{3} f_{2}^{1 \beta}\right\|^{2}-\frac{1}{9} f_{2}^{1 \beta} \cdot f_{4}^{1 \beta}+\frac{1}{9} f_{1}^{\beta \gamma} \cdot f_{4}^{1 \beta}=-4 \text {, } \\
& E_{2,1 \beta}: \quad-4\left\|\frac{1}{3} f_{2}^{1 \beta}\right\|^{2}+\frac{2}{9} f_{2}^{1 \beta} \cdot f_{4}^{1 \beta}+\sum_{\gamma} \frac{1}{6} f_{1}^{\beta \gamma} \cdot\left(\frac{2}{3} f_{2}^{1 \beta}-\frac{1}{3} f_{4}^{1 \beta}\right)=-4, \\
& E_{3,1 \gamma}: \quad-4\left\|\frac{1}{2} f_{3}^{1 \gamma}\right\|^{2}+\frac{1}{3} \sum_{\beta} f_{3}^{1 \gamma} \cdot f_{4}^{1 \beta}=-4, \\
& E_{4,1 \beta}: \quad\left\|\frac{1}{3} f_{2}^{1 \beta}\right\|^{2}+\sum_{\gamma}\left[\left\|\frac{1}{6} f_{1}^{\beta \gamma}\right\|^{2}-\frac{1}{18} f_{1}^{\beta \gamma} \cdot f_{2}^{1 \beta}+\left\|\frac{1}{2} f_{3}^{1 \gamma}\right\|^{2}\right]=12,
\end{aligned}
$$

where we used (3.39), (3.37), the intersection numbers (3.35) and (3.36), $\operatorname{tr}\left(V_{1} V_{2}\right)=\left(V_{1} \cdot V_{2}\right)$ and the definition

$$
(v ; w):=\|v\|^{2}+\|w\|^{2}-v \cdot w .
$$

As we shall see, solving these conditions puts severe constraints on the fluxes.

Eq. (3.42) limits the form of the coefficients of $E_{3,1 \gamma}$ and $E_{3,2 \gamma}$ since it is a sum of positive norms. If one also considers the constraints (3.40), the minimal value of such norms is $3 / 2$ which, in fact, saturates the sum. The possibilities for the coefficients of $f_{3}$ are quite restricted,

$$
\frac{1}{2} f_{3}=\left( \pm \frac{1}{2}, \mp \frac{1}{2}, 0^{6}\right)(\cdots \pm 1 \cdots)
$$

where the notation means that in the second $E_{8}$ the flux can only have one coefficient equal to +1 or -1 , while all the others must be equal to zero.

The condition (3.43) requires for $\left(\frac{1}{3} f_{2} ; \frac{1}{3} f_{4}\right)$ the minimal value $8 / 3$, which again saturates the sum. Therefore we consider

$$
\begin{aligned}
& \frac{1}{3} f_{2}=\left(0,0, \frac{2}{3}, 0^{5}\right)\left(\frac{2}{3}, 0^{7}\right)+\left(x_{1}, \ldots, x_{8}\right)\left(x_{9}, \ldots, x_{16}\right) \\
& \frac{1}{3} f_{4}=\left(0,0,-\frac{2}{3}, 0^{5}\right)\left(-\frac{2}{3}, 0^{7}\right)+\left(y_{1}, \ldots, y_{8}\right)\left(y_{9}, \ldots, y_{16}\right) .
\end{aligned}
$$

When all $x_{i}$ and $y_{i}$ are equal to zero, we have $\left(\frac{1}{3} f_{2} ; \frac{1}{3} f_{4}\right)=\frac{8}{3}$. This value is also realized when $x_{3}$ or $x_{9}$ is equal to -1 and/or at least one of the $y_{3}, y_{9}$ is equal to +1 . Because of $(3.40)$, we want $\left(x_{1}, \ldots x_{8}\right)\left(x_{9}, \ldots, x_{16}\right)$ and $\left(y_{1}, \ldots, y_{8}\right)\left(y_{9}, \ldots, y_{16}\right)$ to be elements of the $E_{8} \times E_{8}$ root lattice. Then, when $x_{3}=-1$, one other coefficient among the $x_{k}$ must be equal to \pm 1 ; the same holds when $x_{9}, y_{3}$ and $y_{9}$ are different from zero. 
Remember that we are only considering fluxes which correspond to the orbifold data in the orbifold limit. The flux along $E_{k}$ can only generate a breaking corresponding to a non-zero VEV of one twisted state in the sector $k$. Moreover, we require that only massless twisted states acquire non-zero VEVs. So the flux coefficient can only be among the shifted momenta of the twisted states given in table 1. This information is crucial for the solution of the Bianchi identities.

Consider now the eq. (3.46). Looking at $k=3$ and $\alpha=1$ in table 1 , we see that the only choices for $f_{3}^{1 \gamma}$ are:

$$
\frac{1}{2} f_{3}^{1 \gamma}=\left( \pm \frac{1}{2}, \mp \frac{1}{2}, 0^{6}\right)\left(1,0^{7}\right),
$$

as the only twisted state is a doublet of $\mathrm{SU}(2)$ with charge +2 with respect to the $\mathrm{U}(1)$ generated by $T_{2}$. This is consistent with the general solution of (3.42). On the other hand, consider $k=4$ and $\alpha=1$. All of the three states are consistent with the constraints on $f_{4}$ given above. The only choice that satisfies eq. (3.46), with $f_{3}^{1 \gamma}$ given in (3.51), is

$$
\frac{1}{2} f_{4}^{1 \beta}=\left(0^{2},-\frac{2}{3}, 0^{5}\right)\left(\frac{1}{3}, \pm 1,0^{6}\right), \quad \forall \beta,
$$

which corresponds to the state $(1,1,1 ; 14)$ in table 1 .

Now we apply analogous considerations to condition (3.44). In the sector $k=2$ and $\alpha=1$, there are only two possible states (both consistent with the constraints on $f_{2}$ ). In the sector $k=1$ and $\alpha=1$ there are four multiplets. Using eq. (3.44), we see that the only states that give a solution belong to the multiplet $(1,1,16 ; 1)$; all the other choices would correspond to fluxes that violate eq. (3.44). Eq. (3.47) is automatically satisfied by this choice, while eq. (3.45) is a linear combination of (3.44) and (3.47).

On the orbifold side, we found a flat direction by giving VEVs to the set of twisted states of table 2. One can check that the Bianchi identities ${ }^{11}$ are solved by fluxes corresponding to the shifted momenta of these twisted states,

$$
\begin{array}{lllll}
\frac{1}{6} f_{1}^{\beta \gamma}=\tilde{p}_{1}^{\beta \gamma} & \forall \beta, \gamma, & \frac{1}{2} f_{3}^{2 \gamma}=\tilde{p}_{3 ;+}^{\gamma} \quad \forall \gamma, \\
\frac{1}{3} f_{2}^{1 \beta}=\tilde{p}_{2 ; 1}^{\beta} \quad \forall \beta, & \frac{1}{3} f_{4}^{1 \beta}=\tilde{p}_{4 ; 1}^{\beta} \quad \text { for } \forall \beta, \\
\frac{1}{3} f_{2}^{3 \beta}=\tilde{p}_{2 ;+}^{\beta} & \forall \beta, & \frac{1}{3} f_{4}^{1 \beta}=\tilde{p}_{4 ;-}^{\beta} & \text { for } \forall \beta, \\
\frac{1}{2} f_{3}^{1 \gamma}=\tilde{p}_{3 ; 1}^{\gamma} & \forall \gamma, & \frac{1}{3} f_{4}^{3 \beta}=\tilde{p}_{4 ;+}^{\beta} \quad \forall \beta ;
\end{array}
$$

here $\tilde{p}_{k ; \alpha}^{\beta \gamma}$ are the orbifold shifted momenta of the twisted states in table 2. Hence, we can indeed match the flux solving the Bianchi identities with the shift vectors of the twisted states whose generic VEVs satisfy the D-term conditions (3.15). Thus the flux (3.53) leaves the following gauge group unbroken

$$
\mathrm{SU}(2) \times \mathrm{SU}(3) \times \mathrm{U}(1)^{5} \times \mathrm{SO}(12) \times \mathrm{U}(1)^{2} .
$$

\footnotetext{
${ }^{11}$ In particular, we see that if we take the anisotropic limit in both directions $\left(K 3_{I I I b} \times T^{2}\right) / \mathbb{Z}_{2}$ and $\left(K 3_{I I b} \times T^{2}\right) / \mathbb{Z}_{3}$, the flux on the cycles that remain large (K3 cycles) is that given in models IIIb and IIb of [10]. Actually the flux in the first case is the one we discussed as the second choice for IIIb.
} 
The above flux is a linear combination of several linearly dependent generators. If we express it in terms of independent generators, it takes the form

$$
F=\sum_{m=1}^{7} f_{m} \mathcal{F}_{m},
$$

where the $f_{m}$ are the generators $T_{1}^{(1)}, T_{1}^{(2)}, T_{1}^{(3)}, T_{2}, T_{3}, \tilde{T}_{2}, \tilde{T}_{4}$ of the seven $\mathrm{U}(1)$ factors in (3.54), given in appendix $\mathrm{C}$, and the $\mathcal{F}_{m}$ are the corresponding coefficients, which are integral two-forms living in the space spanned by the Poincaré dual of the exceptional divisors $E_{k}$.

Using this expression we have checked that the flux also satisfies the K-theory constraints

$$
\sum_{m} c_{1}\left(L_{m}\right)=\sum_{m} \mathcal{F}_{m} \in H^{2}(T, 2 \mathbb{Z})
$$

which says that the second Stiefel-Whitney class of the gauge bundle has to vanish [44-47] (see also ref. [48] for a review).

\subsection{Geometric moduli vs twisted states}

Let us now determine the correspondence between the twisted states of the orbifold background and geometric moduli which govern the size of cycles that are blown up when moving away from the orbifold locus. If these states are flat directions one can blow up the orbifold completely and arrive at a smooth Calabi-Yau space.

As explained in refs. $[14,15]$, for a given choice of flux one can identify the twisted states which have non-zero VEV. The prescription is the following: Take the flux (3.39) that is a linear combination of the (Poincaré dual of the) exceptional divisors. Their coefficients are vectors in the weight space of $E_{8} \times E_{8}$. The twisted state in the twisted sector $k$ that takes a non-zero VEV is the one that has the shifted momentum equal to the coefficient $f_{k}$ of the exceptional divisor $E_{k}$. It is then straightforward to identify the twisted Kähler moduli $t_{E_{k}}$ using the flux (3.39) with $f_{k}$ given by (3.53). The $t_{E_{k}}$ are the volumes of the 32 twisted states shown in table 4 .

In addition we also want to identify the complex structure moduli. In a smooth background with Abelian fluxes the complex structure moduli are not fixed. Therefore we look for flat directions among the twisted states on the orbifold side. In our example we have 10 "twisted" complex structure moduli. From our treatment of the resolution of the $T^{6} / \mathbb{Z}_{6-I I}$ orbifold through two steps, we know that six of them are the complex structure moduli of K3 that survive the $\mathbb{Z}_{2}$ projection, and the remaining four come from the complex structure deformations of the four-cycles arising after the blow-up of the $\mathbb{Z}_{2}$ singularities (see discussion below eq. (3.32)). Since the holomorphic two-form K3 is odd under $\mathbb{Z}_{2}$ its deformation must be chosen from odd combinations of the twisted sectors corresponding to $\alpha=3$ and $\alpha=5$. These deformations are related to the twisted geometric moduli of the resolved $T^{4} / \mathbb{Z}_{3}$ and hence, they must be in the $k=2$ and $k=4$ sectors in table 1, labeled by $\alpha=3-5$.

The remaining four twisted complex structure moduli must be in the $k=3$ sector. We can identify them by considering the orbifold $T^{6} / \mathbb{Z}_{6-I I}$ as $\left(T^{4} / \mathbb{Z}_{2} \times T^{2}\right) / \mathbb{Z}_{3}$. In this case 


\begin{tabular}{|c|c|c|c|c|c|c|c|}
\hline & Multiplet & $k$ & $\alpha$ & $\beta$ & $\gamma$ & $T_{1}$ & $T_{2}$ \\
\hline \multirow{4}{*}{$\begin{array}{r}t_{E_{1, \beta \gamma}} \hookrightarrow \\
\\
\\
\end{array}$} & $(1,1,16 ; 1)$ & 1 & 1 & $1,2,3$ & $1,2,3,4$ & $-1 / 3$ & $2 / 3$ \\
\hline & $(2,1,1 ; 1)$ & 1 & 1 & $1,2,3$ & $1,2,3,4$ & $2 / 3$ & $2 / 3$ \\
\hline & $(2,1,1 ; 1)$ & 1 & 1 & $1,2,3$ & $1,2,3,4$ & $2 / 3$ & $2 / 3$ \\
\hline & $(1,2,1 ; 1)$ & 1 & 1 & 2,3 & $1,2,3,4$ & $-4 / 3$ & $2 / 3$ \\
\hline \multicolumn{2}{|c|}{$t_{E_{2,1 \beta}} \hookrightarrow(1,1,1 ; 1)$} & 2 & 1 & $1,2,3$ & - & $4 / 3$ & $4 / 3$ \\
\hline & $(2,2,1 ; 1)$ & $z^{2}$ & 1 & $1,2,3$ & - & $-2 / 3$ & $4 / 3$ \\
\hline \multirow[t]{2}{*}{$t_{E_{2,3 \beta}}$} & $(1,1,1 ; 1)$ & 2 & $3+5$ & $1,2,3$ & - & $4 / 3$ & $4 / 3$ \\
\hline & $(2,2,1 ; 1)$ & 2 & $3+5$ & $1,2,3$ & - & $-2 / 3$ & $4 / 3$ \\
\hline \multirow{3}{*}{$\zeta_{1}^{3 \beta} \hookrightarrow($} & $(1,1,1 ; 1)$ & 2 & $3-5$ & 2,3 & - & $4 / 3$ & $4 / 3$ \\
\hline & $(1,1,10 ; 1)$ & 2 & $3-5$ & $1,2,3$ & - & $-2 / 3$ & $4 / 3$ \\
\hline & $(1,1,1 ; 14)$ & 2 & $3-5$ & $1,2,3$ & - & $4 / 3$ & $-2 / 3$ \\
\hline \multirow{3}{*}{\multicolumn{2}{|c|}{$\begin{aligned} & (1,1,1 ; 1) \\
& (1,1,10 ; 1) \\
t_{E_{4,1 \beta}} \hookrightarrow & (1,1,1 ; 14)\end{aligned}$}} & 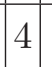 & 1 & 2,3 & - & $-4 / 3$ & $-4 / 3$ \\
\hline & & 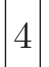 & 1 & $1,2,3$ & - & $2 / 3$ & $-4 / 3$ \\
\hline & & 4 & 1 & $1,2,3$ & - & $-4 / 3$ & $2 / 3$ \\
\hline \multicolumn{2}{|c|}{$\begin{aligned} & t_{E_{4,1 \beta}} \hookrightarrow(1,1,1 ; 14) \\
&(1.1 .1: 1)\end{aligned}$} & 4 & $3+5$ & $1,2,3$ & - & $-4 / 3$ & $-4 / 3$ \\
\hline \multirow{2}{*}{\multicolumn{2}{|c|}{$\begin{array}{r}(1,1,10 ; 1) \\
t_{E_{4,3 \beta} \hookrightarrow}(1,1,1 ; 14)\end{array}$}} & ${ }^{4}$ & $3+5$ & $1,2,3$ & - & $2 / 3$ & $-4 / 3$ \\
\hline & & 4 & $3+5$ & $1,2,3$ & - & $-4 / 3$ & $2 / 3$ \\
\hline \multicolumn{2}{|c|}{$\zeta_{2}^{3 \beta} \hookrightarrow(1,1,1 ; 1)$} & 4 & $3-5$ & $1,2,3$ & - & $-4 / 3$ & $-4 / 3$ \\
\hline \multicolumn{2}{|c|}{$(2,2,1 ; 1)$} & 4 & $3-5$ & $1,2,3$ & - & $2 / 3$ & $-4 / 3$ \\
\hline \multicolumn{2}{|c|}{$t_{E_{3,1 \gamma}} \hookrightarrow(1,2,1 ; 1)$} & 3 & 1 & - & $1,2,3,4$ & 0 & 2 \\
\hline \multicolumn{2}{|c|}{$t_{E_{3,2 \gamma}} \hookrightarrow(1,2,1 ; 1)$} & 3 & $2+4+6$ & - & $1,2,3,4$ & 0 & 2 \\
\hline \multicolumn{2}{|c|}{$\zeta^{2 \gamma} \hookrightarrow(1,2,1 ; 1)$} & 3 & $2+\nu 4+\nu^{2} 6$ & - & $1,2,3,4$ & 0 & -2 \\
\hline & $(1,2,1 ; 14)$ & & $2+\nu^{2} 4+\nu^{4} 6$ & - & $1,2,3,4$ & 0 & 0 \\
\hline
\end{tabular}

Table 4. Geometric moduli among the twisted states of the massless orbifold spectrum. 32 states are identified as volume moduli $t_{E_{k}}$ of the corresponding exceptional divisors $E_{k} ; \zeta_{1}^{3 \beta}, \zeta_{2}^{3 \beta}$ and $\zeta_{1}^{2 \gamma}$ denote 10 complex structure moduli.

$T^{4} / \mathbb{Z}_{2}$ is blown up to a smooth $\widetilde{K 3}$ and one should find a $\mathbb{Z}_{3}$ symmetry of $\widetilde{K 3}$ to take the last quotient. This is more difficult than finding an involution and we will not study this procedure in detail. On the other hand, by analogy with the $T^{4} / \mathbb{Z}_{3}$ case, we can make an educated guess. In fact the holomorphic two-form $\Omega_{\widetilde{K 3}}$ should transform with a phase under the $\mathbb{Z}_{3}$ and therefore its deformation should exhibit the same phase. The states with this property are labeled by $\alpha=2+\nu 4+\nu^{2} 6$ and $\alpha=2+\nu^{2} 4+\nu^{4} 6$ in table 1 . Depending 
on the choice of the complex structure it will be one of the two states. ${ }^{12}$ The matching of the geometric moduli with the twisted states is summarized in table 4 .

\subsection{The spectrum on the smooth Calabi-Yau}

The flux given in eq. (3.53) induces the following breaking of the gauge group

$$
E_{8} \times E_{8} \quad \rightarrow \quad \mathrm{SU}(2) \times \mathrm{SU}(3) \times \mathrm{U}(1)^{5} \times \mathrm{SO}(12) \times \mathrm{U}(1)^{2} .
$$

The adjoint representations of the two $E_{8}$ factors decompose according to

$$
\begin{aligned}
& \mathbf{2 4 8}^{(1)}=(\mathbf{1}, \mathbf{3}) \oplus(\mathbf{8}, \mathbf{1}) \oplus(\mathbf{1}, \mathbf{1})^{5} \oplus\left(\mathbf{R}^{(\mathbf{1})} \oplus \overline{\mathbf{R}}^{(\mathbf{1})}\right), \\
& \mathbf{2 4 8}^{(2)}=\mathbf{6 6} \oplus \mathbf{1} \oplus \mathbf{1} \oplus\left(\mathbf{R}^{(\mathbf{2})} \oplus \overline{\mathbf{R}}^{(\mathbf{2})}\right),
\end{aligned}
$$

where the entries in the first line correspond to $\mathrm{SU}(2) \times \mathrm{SU}(3)$ while the second decomposition is with respect to $\mathrm{SO}(12)$. $\mathbf{R}^{(\mathbf{1})}$ and $\mathbf{R}^{(\mathbf{2})}$ denote reducible representation of the unbroken gauge group. The irreducible representations in $\mathbf{R}^{(\mathbf{1})}$ and in $\mathbf{R}^{(\mathbf{2})}$ are listed in appendix $\mathrm{C}$ (tables 10 and 11). Since they are charged under the fluxed $\mathrm{U}(1) \mathrm{s}$, there can be chiral states in these representation. The chiral multiplicity is given by an index theorem (see for example [49]) . Following [12, 14], we can use the "number operator"

$$
N=\frac{1}{6} \int_{X}\left(F \wedge F \wedge F+\frac{1}{2} F \wedge c_{2}(X)\right),
$$

to compute the spectrum. The eigenspaces of $N$ are the representations of the unbroken gauge group and the corresponding eigenvalues give the multiplicity of these representations. Note that tracing the expression (3.59) over the representation $\mathbf{R}$ gives the index of the Dirac operator index $\mathbf{R}_{\mathbf{R}} \not D$ in that representation.

Inserting the expression for the flux (3.39) into (3.59), one obtains:

$$
\begin{aligned}
N= & \frac{4}{3} \sum_{\beta \gamma}\left(V_{1}^{\beta \gamma}\right)^{3}+\frac{4}{3} \sum_{\beta}\left(V_{2}^{1 \beta}\right)^{3}+\frac{4}{3} \sum_{\gamma}\left(V_{3}^{1 \beta \gamma}\right)^{3}-\sum_{\beta \gamma} V_{1}^{\beta \gamma}\left(V_{2}^{1 \beta}\right)^{2}-\sum_{\beta \gamma}\left(V_{1}^{\beta \gamma}\right)^{2} V_{4}^{1 \beta} \\
& -\sum_{\beta}\left(V_{2}^{1 \beta}\right)^{2} V_{4}^{1 \beta}+\sum_{\beta \gamma} V_{1}^{\beta \gamma} V_{2}^{1 \beta} V_{4}^{1 \beta}-\sum_{\beta \gamma}\left(V_{3}^{1 \gamma}\right)^{2} V_{4}^{1 \beta} \\
& -\frac{1}{3} \sum_{\beta \gamma} V_{1}^{\beta \gamma}-\frac{1}{3} \sum_{\beta} V_{2}^{1 \beta}-\frac{1}{3} \sum_{\gamma} V_{3}^{1 \gamma}+\sum_{\beta} V_{4}^{1 \beta} .
\end{aligned}
$$

The $V_{i}^{\beta \gamma}$ are elements of the Cartan subalgebra of $E_{8}$. They act on an element of the adjoint representation of $E_{8}$ labeled by the root $\alpha$ as

$$
\left[V_{i}^{\beta \gamma}, v_{\alpha}\right]=\left(\alpha \cdot V_{i}^{\beta \gamma}\right) v_{\alpha} .
$$

Then, if we know the roots corresponding to the decompositions of the adjoint of $E_{8} \times E_{8}$, we are able to compute the multiplicity of the given representation. We find

$$
V_{1}^{\beta \gamma}=\frac{1}{6} f_{1}^{\beta \gamma}, \quad V_{2}^{1 \beta}=\frac{1}{3} f_{2}^{1 \beta}, \quad V_{3}^{1 \gamma}=\frac{1}{2} f_{3}^{1 \gamma}, \quad V_{4}^{1 \beta}=\frac{1}{3} f_{4}^{1 \beta} .
$$

\footnotetext{
${ }^{12}$ With our choice of the orbifold action given in (3.3) the $T^{4} / \mathbb{Z}_{2}$ in question is along $z_{1}, z_{3}$. In the singular limit, the holomorphic two-form is $\Omega_{\tilde{K} 3} \sim d z_{1} \wedge d z_{3}$ and transforms under $\mathbb{Z}_{3}$ as $\Omega_{\tilde{K} 3} \mapsto e^{2 \pi i / 3} \Omega_{\tilde{K} 3}$. This is the transformation of the states labeled by $\alpha=2+\nu 4+\nu^{2} 6$, where $\nu=e^{-2 \pi i / 3}$.
} 
The $V_{i}^{\beta \gamma} \mathrm{s}$ can be written in terms of the generators of the $\mathrm{U}(1)$ factors in the unbroken gauge group. Then the eigenvalues of the number operator are given in terms of the charges of the representation $\mathbf{R}_{i}$ with respect to the $\mathrm{U}(1)$ factors. The detailed computation of the chiral spectrum is given in appendix $\mathrm{C}$.

Switching on the flux (3.55) renders all the seven U(1) factors in (3.57) massive with a Stückelberg induced mass matrix (see e.g. [48] for a review),

$$
M_{m n}^{2}=\sum_{j} \frac{1}{\alpha_{j}} \mathcal{M}_{j m} \mathcal{M}_{j n}
$$

where $\alpha_{j}$ and $\mathcal{M}_{j m}$ appear in the axion part of the $4 \mathrm{D}$ effective action,

$$
S \subset \sum_{j=0}^{h^{1,1}} \int_{\mathbb{R}^{1,3}} b_{j}^{2} \wedge \sum_{m} \mathcal{M}_{j m} F_{m}+\alpha_{j} \int_{\mathbb{R}^{1,3}} d b_{j}^{2} \wedge * d b_{j}^{2} .
$$

$F_{m}$ is the field strength of an unbroken $\mathrm{U}(1)$ factor and in our case $m$ takes the values $m=1, \ldots, 7 . b_{j}^{2}$ are the $4 \mathrm{D}$ axionic two-forms coming from the expansion of $B_{6}$ along the harmonic four-form of the Calabi-Yau, plus the universal two-form $B_{2}$ along the $4 \mathrm{D}$ spacetime. (Dualizing these two forms one gets the axionic scalars). We neglect the couplings $\mathcal{M}_{0 m}$ related to the universal axion and only consider

$$
\mathcal{M}_{j m}=\sum_{n=1}^{7}\left(f_{m} \cdot f_{n}\right) \int_{Y} \mathcal{F}_{n} \wedge \eta_{j},
$$

where $\left\{\eta_{j}\right\}$ is a basis of $H^{4}(Y), f_{n}$ are elements of the Cartan subalgebra of $E_{8} \times E_{8}$ and $\mathcal{F}_{n} \in H^{2}(Y, \mathbb{Z})$ is such that the flux is given by (3.55). One observes that with our choice of flux the matrix $\mathcal{M}_{j m}$ has maximal $\operatorname{rank} \operatorname{rk}\left(\mathcal{M}_{j m}\right)=7$. This implies that all the seven $\mathrm{U}(1)$ factors become massive due to the presence of the flux and the unbroken gauge group reduces to

$$
\mathrm{SU}(2) \times \mathrm{SU}(3) \times \mathrm{SO}(12) .
$$

This is indeed the same gauge group obtained on the orbifold side on the Higgs branch.

Note that the spectrum given in tables 10 and 11 is not chiral with respect to the surviving gauge group (3.66). This makes the identification of the states more difficult than in the $6 \mathrm{D}$ case. To match the two spectra, we still have to consider the charges under the massive U(1)s. This forces us to redefine the states on the orbifold side, using the singlets that acquire a VEV (see [13]).

Matching with the Higgsed orbifold spectrum. The spectrum on the smooth side does not match with the one at the orbifold point. In fact a smooth compactification corresponds to switching on VEVs for the twisted states which correspond to the geometric moduli. This breaks the gauge group at the orbifold locus to the subgroup which is visible on the smooth side via the Higgs mechanism. Some of the chiral multiplets are eaten by the massive gauge bosons. Moreover, to achieve a match of the $\mathrm{U}(1)$ charges, it is necessary to redefine the fields on the orbifold side as we did in eq. (2.16). In the $T^{6} / \mathbb{Z}_{6}$ case this 


\begin{tabular}{|c|c|ccccccc|}
\hline & & $q_{1}^{(1)}$ & $q_{1}^{(2)}$ & $q_{1}^{(3)}$ & $q_{2}$ & $q_{3}$ & $\tilde{q}_{2}$ & $\tilde{q}_{4}$ \\
\hline 1 & $(3,1 ; 1)$ & $-13 / 3$ & $5 / 3$ & $5 / 3$ & 0 & $-1 / 3$ & $2 / 3$ & $1 / 3$ \\
2 & $(3,1 ; 1)$ & $5 / 3$ & $-1 / 3$ & $5 / 3$ & 0 & $-1 / 3$ & $2 / 3$ & $1 / 3$ \\
3 & $(\overline{3}, 1 ; 1)$ & $5 / 3$ & $5 / 3$ & $5 / 3$ & 0 & $-1 / 3$ & $2 / 3$ & $1 / 3$ \\
4 & $(\overline{3}, 1 ; 1)$ & $5 / 3$ & $5 / 3$ & $-1 / 3$ & 0 & $-1 / 3$ & $2 / 3$ & $1 / 3$ \\
5 & $(1,2 ; 1)$ & $-1 / 3$ & $-1 / 3$ & $-1 / 3$ & 0 & $2 / 3$ & $2 / 3$ & $1 / 3$ \\
6 & $(1,2 ; 1)$ & $-1 / 3$ & $-1 / 3$ & $-1 / 3$ & 0 & $2 / 3$ & $2 / 3$ & $1 / 3$ \\
7 & $(1,1 ; 1)$ & $2 / 3$ & $5 / 3$ & $-1 / 3$ & 0 & $-1 / 3$ & $2 / 3$ & $1 / 3$ \\
8 & $(1,1 ; 1)$ & $5 / 3$ & $2 / 3$ & $-1 / 3$ & 0 & $-1 / 3$ & $2 / 3$ & $1 / 3$ \\
9 & $(1,1 ; 1)$ & $2 / 3$ & $2 / 3$ & $2 / 3$ & 1 & $-4 / 3$ & $2 / 3$ & $1 / 3$ \\
10 & $(1,1 ; 1)$ & $2 / 3$ & $2 / 3$ & $2 / 3$ & -1 & $-4 / 3$ & $2 / 3$ & $1 / 3$ \\
11 & $(1,1 ; 1)$ & $-1 / 3$ & $-1 / 3$ & $2 / 3$ & 0 & $-1 / 3$ & $2 / 3$ & $1 / 3$ \\
12 & $(1,1 ; 1)$ & $-13 / 3$ & $-13 / 3$ & $5 / 3$ & 0 & $-1 / 3$ & $2 / 3$ & $1 / 3$ \\
\hline
\end{tabular}

Table 5. The 22 states of the twisted sector $k=1$; states from the representation $(1,1,16 ; 1)$ acquire a VEV, e.g. state \#12.

is slightly more difficult than for the $T^{6} / \mathbb{Z}_{3}$ orbifolds discussed in $[13,16]$. The reason is that states in different twisted sectors can mix due to the fact that for $T^{6} / \mathbb{Z}_{6}$ the singular loci intersect each other. We have been able to find field redefinition which match the charged states on both sides. For the singlets however, the possible redefinitions are less constrained and therefore we do not attempt to match them in this paper. We do not present the complete computation here but as a representative example go through the sector $k=1$ with $\alpha=1, \beta=1, \gamma=1$. The spectrum after higgsing is given in table 5 . The charges refer to the $\mathrm{U}(1) \mathrm{s}$ along which the flux is switched on in the smooth case. The field that gets non-zero VEV is \#12, the last one in table 5 , which corresponds to the one in the first line of table 2 . We call it $\Phi_{1}$. It has the following shifted vector:

$$
\tilde{p}_{1,1}=\left(0^{2},-\frac{1}{6},-\frac{1}{2},-\frac{1}{2},-\frac{1}{2},-\frac{1}{2},-\frac{1}{2}\right)\left(\frac{1}{3}, 0^{7}\right) .
$$

To redefine the fields in the sector $k=1, \alpha=1, \beta=1, \gamma=1$, the field $\Phi_{1}$ is not enough to get the right match with the spectrum on the smooth side. We need to use fields from other twisted sectors that live on subspaces intersecting the point-like singularity where the states (5) live. These are the twisted states that take non-zero VEV in the other sector after the blow-up. For the chosen twisted sector, in addition to the field $\Phi_{1}$ we need to consider also the field $\Phi_{2}$ from the sector $k=2, \alpha=1, \beta=1$, with shifted vector

$$
\tilde{p}_{21}=\left(0,0, \frac{2}{3}, 0,0,0,0,0\right)\left(\frac{2}{3}, 0,0^{6}\right) .
$$




\begin{tabular}{|c|c|ccccccc|}
\hline & & $q_{1}^{(1)}$ & $q_{1}^{(2)}$ & $q_{1}^{(3)}$ & $q_{2}$ & $q_{3}$ & $\tilde{q}_{2}$ & $\tilde{q}_{4}$ \\
\hline 1 & $(3,1 ; 1)$ & 4 & 4 & -2 & 0 & -2 & 0 & 0 \\
2 & $(3,1 ; 1)$ & -6 & -6 & 6 & 0 & 0 & 0 & 0 \\
3 & $(\overline{3}, 1 ; 1)$ & -6 & 0 & 6 & 0 & 0 & 0 & 0 \\
4 & $(\overline{3}, 1 ; 1)$ & -6 & 0 & 0 & 0 & 0 & 0 & 0 \\
5 & $(1,2 ; 1)$ & -8 & -2 & 4 & 0 & 0 & 0 & 0 \\
6 & $(1,2 ; 1)$ & -8 & -2 & 4 & 0 & 0 & 0 & 0 \\
7 & $(1,1 ; 1)$ & 0 & 0 & 0 & 0 & 0 & 0 & 0 \\
8 & $(1,1 ; 1)$ & -6 & 6 & 0 & 0 & 0 & 0 & 0 \\
9 & $(1,1 ; 1)$ & -7 & -1 & 5 & 1 & -1 & 0 & 0 \\
10 & $(1,1 ; 1)$ & -7 & -1 & 5 & 1 & 1 & 0 & 0 \\
11 & $(1,1 ; 1)$ & 4 & -2 & 4 & 0 & -2 & 0 & 0 \\
12 & $(1,1 ; 1)$ & 4 & -2 & -2 & 0 & -2 & 0 & 0 \\
\hline
\end{tabular}

Table 6. The 22 states on the smooth side, which correspond to the states of the twisted sector $k=1$.

In particular, we redefine the first and the last two states in table 5 multiplying them by $\Phi_{1} \Phi_{2}^{-1}$ and the other states by $\Phi_{1}^{-1}$. The charges of the new fields are given in table 6 . We end up with fields that now are charged only under one $E_{8}$ and can therefore be matched with the fields in the smooth sector. The states \#2,3,4,8 have a complex conjugate partner (with respect to the massive $\mathrm{U}(1) \mathrm{s})$ coming from other $k=1$ sectors, and then decouple from the spectrum. The state \#7 is the geometric modulus corresponding to the size of the blown up four-cycle. The other states can be recognized to be among the states in table 6. To have the right multiplicities, one has to consider the complete orbifold higgsed spectrum. For example, states like state $\# 1$ in table 6 also appear in the sectors $k=1$, $\alpha=1, \beta=1,2,3, \gamma=1,2$, with total multiplicity 6 . It also appears with opposite chirality (with respect to the massive $\mathrm{U}(1) \mathrm{s}$ ), in the untwisted spectrum with multiplicity 1 , and in the twisted $k=4, \alpha=1, \beta=1,2,3$ with multiplicity 3 . The total number of chiral states in this representation is $n=2$, that matches with the multiplicity given in the table 10 . The same happens for all the fields in the non-trivial representations of the unbroken group.

\section{Conclusions}

We have studied the relation between a specific orbifold background and a smooth CalabiYau compactification which contains the orbifold background as a singular locus in its moduli space. Specifically, we considered the orbifold $T^{6} / \mathbb{Z}_{6-I I}=\left(T^{4} / \mathbb{Z}_{3} \times T^{2}\right) / \mathbb{Z}_{2}$ for which we performed a two-step resolution. As a first intermediate step we resolved $T^{4} / \mathbb{Z}_{3}$ to a smooth $K 3$ surface. For the compactification on K3 to six dimensions we discussed 
the line bundle corresponding to the chosen $\mathbb{Z}_{3}$ orbifold gauge twist. We also identified the twisted states which correspond to the geometric $K 3$ moduli. After giving VEVs to these states, the orbifold gauge group and its massless spectrum matched the gauge group and spectrum of the smooth compactification.

In the second step we compactified to four dimensions and studied the resolution of the singular space $Y_{s}=\left(K 3 \times T^{2}\right) / \mathbb{Z}_{2}$. In particular, finding how the $\mathbb{Z}_{2}$ involution acts on the $\mathrm{K} 3$ factor, we were led to consider heterotic compactifications on a specific smooth Voisin-Borcea manifold. This manifold is a K3 fibration and in the limit of a large $\mathbb{P}^{1}$-base one recovers the $6 \mathrm{D}$ compactifications on K3. The considered Voisin-Borcea manifold has a specific intersection form which can be compared with the toric resolutions studied in [14]. The latter come from different triangulations that one can perform at each singularity of $T^{6} / \mathbb{Z}_{6-I I}$. With the help of this comparison we were able to identify which triangulation corresponds to our specific Voisin-Borcea manifold.

In heterotic compactifications it is necessary to also identify the gauge bundle which solves the Bianchi identities. Here we considered non-trivial line bundles (fluxes) on the Voisin-Borcea manifold that in the orbifold limit reproduce the gauge twists up to vectors of the $E_{8} \times E_{8}$ root lattice. It turned out that the fluxes are severely constrained by the Bianchi identities. In particular, we found that some flux on the K3 fiber allowed in the 6D background, no longer could be turned on. The resulting low energy gauge group is small, even smaller than the standard model gauge group. We could show that for the unbroken group the charged massless states match. However, the massless spectrum is non-chiral with respect to the unbroken gauge group. Hence, we do not obtain the phenomenologically interesting orbifold background as limit of a related smooth CY compactification.

This result, in particular the breaking of the phenomenologically interesting $\mathrm{SO}(10)$ GUT group down to $\mathrm{SU}(3)$, came as a surprise, and it is clearly important to understand its origin. The matching conditions between fluxes and orbifold gauge twists involve $E_{8} \times E_{8}$ root vectors which are related to the shifted momenta of twisted orbifold states acquiring non-zero VEVs. We only found solutions of the Bianchi identities where 16-plets of $\mathrm{SO}(10)$ obtain VEVs. This immediately implies a strong breaking of the orbifold gauge group. It is conceivable that we missed interesting solutions or that new solutions appear once discrete Wilson lines are turned on. A further possibility is that the employed matching conditions, which so far represent a conjecture, are too restrictive. In [14], the MSSM gauge group was found, but only at the expense of assigning VEVs to massive twisted states which cannot represent moduli. For the toy model with standard embedding in [15], a complete blow up was achieved by giving VEVs to massless twisted states only. However, the unbroken group does not contain the standard model gauge group and the massless spectrum is non-chiral. Note that in this paper we have only studied a particular example and that for other blown-up orbifolds chiral spectra may very well exist. Clearly, further studies are needed to clarify the situation, and one may also consider the case of partial blow-up.

Our study also illustrates some conceptual problems which one faces in attempts to embed the standard model into string theory. In orbifold compactifications one constructs massless states of the free string at a particular point in moduli space. Information about the couplings of twisted states located at different singularities is only contained in the 
D-terms of the unbroken 4D gauge group. For the considered orbifold, some information on the superpotential is provided by string selection rules, but not enough is known to rigorously check the vanishing of the F-terms. On the other hand, a detailed knowledge of the superpotential is required to demonstrate the decoupling of the vector-like exotics. Realistic models require to go away from the orbifold point by assigning large VEVs to twisted states, and at present one cannot be sure whether the resulting gauge group and spectrum really correspond to a string vacuum.

On the other hand, in Calabi-Yau compactifications one starts from the supergravity approximation in ten dimensions. The geometry of the compact space then provides valuable information on possible breakings of the $E_{8} \times E_{8}$ gauge group and the related massless spectrum via the Bianchi identities. However, since one is dealing with supergravity rather than string theory, the singular orbifold limit cannot be performed and one can only compare gauge group and massless spectrum with an orbifold construction where part of the gauge group is higgsed. There are intriguing relations between CY fluxes and orbifold twisted states, but the precise matching conditions are presently unclear.

Embedding the (supersymmetric) standard model into string theory is a challenging problem, even before addressing the question of supersymmetry breaking. In the long run, the matching between orbifold constructions and supergravity compactifications on Calabi-Yau manifolds may become a useful tool. The orbifold constructions can provide some intuition to identify interesting geometries and fluxes, which yield the wanted chiral massless spectrum. The corresponding smooth compactifications may then help to understand the dynamical stabilization of the ground state.

\section{Acknowledgments}

We would like to thank Martin Schasny for helpful discussions, and Stefan Groot Nibbelink and Patrick Vaudrevange for correspondence and a careful reading of the manuscript. This work has been supported by the German Science Foundation (DFG) within the Collaborative Research Center 676 "Particles, Strings and the Early Universe" and the Research Training Group 1670.

\section{A Geometric details}

In this appendix we supplement the main text with additional material about K3. Let us start by discussing non-symplectic involutions on K3 surfaces as used in section 3.2.

\section{A.1 Non-symplectic involutions on K3 surfaces}

A holomorphic involution of K3 is called non-symplectic when it reverses the sign of the holomorphic two-form $\Omega$. These involutions have been studied and classified by Nikulin [39$42]$, and we review here some of their properties.

The non-symplectic involutions $\sigma$ of K3 are classified in terms of three parameters: $(r, a, \delta)$, which characterize the lattice $S_{+}$of even cycles which is a sublattice of the Picard 
lattice (as $\left.\Omega_{2} \mapsto-\Omega_{2}\right) \cdot{ }^{13} r$ is the rank of $S_{+}$while $a$ is defined by the expression $S_{+}^{*} / S_{+}=$ $\left(\mathbb{Z}_{2}\right)^{a} \cdot{ }^{14} \delta$ can take two values: $\delta=0$ if $\forall(x, \cdot) \in S_{+}^{*}(x, x) \in \mathbb{Z}, \delta=1$ otherwise.

The triplet of parameters $(r, a, \delta)$ encodes information about the fixed point set in K3. If $(r, a, \delta) \neq(10,10,0)$ and $(r, a, \delta) \neq(10,8,0)$, then the fixed point set is given by

$$
\bigcup_{i=1}^{k} \mathbb{P}_{i}^{1} \cup C_{g},
$$

i.e., it is a disjoint union of $k$ rational curves (the $\mathbb{P}^{1} \mathrm{~s}$ ) and one curve $C_{g}$ with genus $g$. $k$ and $g$ are in turn determined by $r$ and $a$ via

$$
k=\frac{1}{2}(r-a), \quad g=\frac{1}{2}(22-r-a) .
$$

When $(r, a, \delta)=(10,10,0)$ the involution acts freely (called Enriques involution), while if $(r, a, \delta)=(10,8,0)$ the fixed point set is a disjoint union of two elliptic curves (two two-tori).

\section{A.2 Blowing up the $A_{2}$-singularities}

Let us now consider the geometric transition from $T^{4} / \mathbb{Z}_{3}$ to a smooth $\mathrm{K} 3$ from an algebraic point of view. The nine $A_{2}$-singularities can be resolved by rotating the Kähler form $j$ into the space of $A_{2}$-cycles such that they are no longer orthogonal to the $\Sigma$ plane and thus have finite size.

From an algebraic point of view, the blow-up is described as follows (see [28] for more details). Every $A_{2}$ singularity is locally of the form $\mathbb{C}^{2} / \mathbb{Z}_{3}$. It is given by modding out $(\xi, \eta) \sim\left(e^{2 \pi i / 3} \xi, e^{-2 \pi i / 3} \eta\right)$ from $\mathbb{C}^{2}$. Let us choose three coordinates invariant under $\mathbb{Z}_{3}$ :

$$
x_{0}=\xi^{3}, \quad x_{1}=\eta^{3}, \quad x_{2}=\xi \eta,
$$

which obey

$$
x_{0} x_{1}-x_{2}^{3}=0
$$

in $\mathbb{C}^{3}$. This space has one singularity located at the origin. One could remove this singularity by adding a term to the polynomial (e.g. by deforming eq. (A.4) into $x_{0} x_{1}-x_{2}^{3}=\epsilon$ ). This would correspond to a complex structure deformation, i.e., to a rotation of $\Omega_{2}$ into the $A_{2}$-cycles which then are of finite size. However, we are interested in a rotation of the Kähler form, while keeping $\Omega_{2}$ orthogonal to the $A_{2}$-cycles. This corresponds to blowing up the singularity. One introduces a $\mathbb{P}^{2}$ parametrized by coordinates $\left\{s_{0}, s_{1}, s_{2}\right\}$, subject to the conditions

$$
x_{0} s_{1}=x_{1} s_{0}, \quad x_{0} s_{2}=x_{2} s_{0}, \quad x_{1} s_{2}=x_{2} s_{1} .
$$

The $s_{i}$ are uniquely determined away from the origin $x_{i}=0$. On the other hand, at the point $x_{i}=0 \forall i$ they are left undetermined. Let us choose the following path approaching

\footnotetext{
${ }^{13}$ The Picard lattice is the orthogonal complement of $\Omega_{2}$ in $H_{2}(K 3, \mathbb{Z})$.

${ }^{14}$ For each lattice $S$, there exists a dual lattice $S^{*}=\operatorname{Hom}(S, \mathbb{Z})$. Each element $\beta \in S^{*}$ can be represented by an element $b \in S \otimes \mathbb{R}$ by identifying $\beta(\cdot)=(b, \cdot)$, where $(\cdot, \cdot)$ is the intersection bilinear form on $S$. It can be shown that for $S$ to be the even lattice under an involution on K3, it must satisfy $S^{*} / S=\left(\mathbb{Z}_{2}\right)^{a}$ for some $a$ (i.e. it must be a 2-elementary lattice).
} 
the origin to find the exceptional divisor: $x_{i}=c_{i} t, t \rightarrow 0$. To be inside the space, the following equation must hold:

$$
c_{0} c_{1}-t c_{2}^{3}=0 \quad \Rightarrow \quad s_{0} s_{1}=s_{2}^{2} c_{2} t
$$

which for $t \rightarrow 0$ becomes

$$
s_{0} s_{1}=0 .
$$

We see that at $x_{i}=0$ there are two $\mathbb{P}^{1}$ 's, $\left\{s_{0}=0\right\}$ and $\left\{s_{1}=0\right\}$, intersecting at the point $\left(x_{0}, x_{1}, x_{2} ; s_{0}, s_{1}, s_{2}\right)=(0,0,0 ; 0,0,1)$. These are the cycles with intersection matrix given in eq. (2.24). Moreover one can see that $\left\{s_{0}=0\right\}$ intersects the plane $\{\xi=0\}$ at the point $\left(x_{0}, x_{1}, x_{2} ; s_{0}, s_{1}, s_{2}\right)=(0,0,0 ; 0,1,0)$ and does not intersect $\{\eta=0\}$, while $\left\{s_{1}=0\right\}$ intersects the plane $\{\eta=0\}$ at the point $\left(x_{0}, x_{1}, x_{2} ; s_{0}, s_{1}, s_{2}\right)=(0,0,0 ; 1,0,0)$ and does not intersect $\{\xi=0\}$.

To summarize, we have an intuitive picture of the smooth K3 which arises from blowing up the orbifold $T^{4} / \mathbb{Z}_{3}$. This orbifold is an elliptic fibration over a $\mathbb{P}^{1}$ with nine $A_{2}$ singularities. Over a generic point on the base, the fiber is a $T^{2}$. On top of three points we have the singular fiber $T^{2} / \mathbb{Z}_{3}$. After the blow-up, the singular fiber becomes a collection of seven (six independent) $\mathbb{P}^{1}$ 's with intersection matrix given by the extended Dynkin diagram of $E_{6}$. At this point of the moduli space we have a large number of algebraic cycles: the holomorphic two-form $\Omega_{2}$ is still orthogonal to the full lattice

$$
P_{X}=\mathcal{U} \oplus\left(-E_{6}\right) \oplus\left(-E_{6}\right) \oplus\left(-E_{6}\right) .
$$

A generic smooth point in the K3 moduli space will have a smaller Picard group. For the case at hand we allow $\Omega_{2}$ to move away from this symmetric point.

\section{A.3 Involution on the resolution of $T^{4} / \mathbb{Z}_{3}$}

As we have just seen, an involution on K3 is known once the lattice of even cycles is known. In the following we want to determine how the $\mathbb{Z}_{2}$ involution on $T^{4} / \mathbb{Z}_{3}$ is promoted to a non-symplectic involution on the smooth K3. To do so we use the known action of the $\mathbb{Z}_{2}$ on the orbifold and from this derive the transformation properties of the K3 cycles. In this way we are able to determine the even lattice $S_{+}$and its properties.

For this purpose, we will view $T^{4} / \mathbb{Z}_{3}$ as a singular K3 that is elliptically fibered over the base $T^{2} / \mathbb{Z}_{3}$. Over the singular point of the base (spanned by $z_{1}$ ) the fiber (spanned by $z_{2}$ ) degenerates to $T^{2} / \mathbb{Z}_{3}$. Let us call $z_{1}^{a}, z_{1}^{b}, z_{1}^{c}$ the points where the singular fiber sits. The two two-tori $\Pi_{1}$ and $\Pi_{2}$ are described in the following way: the first is just a point in the base of the elliptic fibration (i.e. it is homologous to the $T^{2}$ fiber), while the second is given by three points in the fiber, exchanged by $\mathbb{Z}_{3}$ (it wraps three times the base). After smoothing the singularities like above, the 18 exceptional $\mathbb{P}^{1}$ 's $e_{\alpha \beta}^{(0)}, e_{\alpha \beta}^{(1)}$ have finite size. The upper index is (1) if the exceptional cycle locally corresponds to $s_{1}=0$ and (0) if it corresponds to $s_{0}=0$.

Let us recall that the $\mathbb{Z}_{2}$ action on coordinates of $T^{4}$ is given by $\left(z_{1}, z_{2}\right) \mapsto\left(-z_{1}, z_{2}\right)$. Then the fiber of the elliptically fibered singular K3 is left invariant, while the $\mathbb{Z}_{2}$ group acts on the base and has two fixed points on it. Under $\mathbb{Z}_{2}$ the locations of the singular fibers behave as follows: $z_{1}^{\alpha=3} \leftrightarrow z_{1}^{\alpha=5}$ while $z_{1}^{\alpha=1}$ is left fixed (see figure 4 ). Let us call the other 
fixed point on the base $z_{1}^{\alpha=2,4,6}$ (on the $T^{2}$ spanned by $z_{1}$ it is given by three points that are exchanged under the $\mathbb{Z}_{3}$ transformation and that then are identified on the $T^{2} / \mathbb{Z}_{3}$ base).

From here we see immediately that the two-cycles corresponding to the fiber and the base of the fibration are even. In particular we have a fixed two-torus over $z_{1}=z_{1}^{\alpha=2,4,6}$. Moreover, the exceptional cycles corresponding to $z_{1}=z_{1}^{\alpha=3}$ are exchanged with the exceptional cycles corresponding to $z_{1}=z_{1}^{\alpha=5}$. To understand how the cycles on $z_{1}^{c}$ behaves we have to use their algebraic description after the blow-up. These cycles are given by the large singular fiber and the six exceptional cycles coming from blowing up the three $\mathbb{C}^{2} / \mathbb{Z}_{3}$ singularities on $z_{1}=z_{1}^{\alpha=1}$. The singular fiber is given by the equation $z_{1}=z_{1}^{\alpha=1}$ and is left fixed by $\mathbb{Z}_{2}$. Let us see the other cycles: In the local coordinates around $z_{1}=z_{1}^{c}$, the action of $\mathbb{Z}_{2}$ is given by $(\xi, \eta) \mapsto(-\xi, \eta)$, where $\xi \leftrightarrow z_{1}$ and $\eta \leftrightarrow z_{2}$. This implies the following transformation on the $x_{i}$ and $s_{i}$ :

$$
\left(x_{0}, x_{1}, x_{2} ; s_{0}, s_{1}, s_{2}\right) \mapsto\left(-x_{0}, x_{1},-x_{2} ; s_{0},-s_{1}, s_{2}\right) .
$$

We recall that the two $\mathbb{P}^{1}$ 's at $x_{i}=0$ are determined by $s_{0}=0$ and $s_{1}=0$. From the transformation above, we see that the first one transforms as the base of the elliptic fibration, i.e., it is even, with two fixed points, while the second is left fixed by $\mathbb{Z}_{2}$. One of the fixed points of $\left\{s_{0}=0\right\}$ is the intersection point with $\left\{s_{1}=0\right\}$, while the other is the intersection point with the singular fiber $(\xi=0)$. As we have seen above, the base $(\eta=0)$ intersects $\left\{s_{1}=0\right\}$, but neither the singular fiber nor $\left\{s_{0}=0\right\}$.

From these considerations we can determine both the even cycles and the fixed point locus. The even cycles are linear combination of:

- the base and the fiber,

- the six exceptional cycles on $z_{1}^{\alpha=1}$,

- the six even combination of the exceptional cycles on $z_{1}^{\alpha=3}$ and $z_{1}^{\alpha=5}$.

As illustrated in figure 4 , the fixed locus is given by

- the two-torus at $z_{1}=z_{1}^{\alpha=2,4,6}$,

- the three $\mathbb{P}^{1} \mathrm{~s} e_{1 \beta}^{(1)}$ at $z_{1}=z_{1}^{c}$ and locally $s=1$.

\section{B 4D orbifold spectrum after higgsing}

Here we list the spectrum given in table 1, after the gauge group is broken to $\mathrm{SU}(2) \times$ $\mathrm{SU}(3) \times \mathrm{SO}(12)$. In the following table we also list the charges of the states with respect to the broken $\mathrm{U}(1)$ generators given in (C.2) and (C.3).

\section{4D spectrum in the smooth case}

In this appendix we compute the spectrum of the heterotic $E_{8} \times E_{8}$ string compactified on the Voisin-Borcea manifold $Y$ which we discussed in section 3.5 together with the choice of the flux given in eqs. (3.39) and (3.53). The unbroken gauge group is

$$
\mathrm{SU}(2) \times \mathrm{SU}(3) \times \mathrm{U}(1)^{5} \times \mathrm{SO}(12) \times \mathrm{U}(1)^{2},
$$




\begin{tabular}{|c|c|c|c|c|c|c|c|c|c|c|c|}
\hline Multiplet & $k$ & $\alpha$ & $\beta$ & $\gamma$ & $q_{1}^{(1)}$ & $q_{1}^{(2)}$ & $q_{1}^{(3)}$ & $q_{2}$ & $q_{3}$ & $\tilde{q}_{2}$ & $\tilde{q}_{4}$ \\
\hline$(1,1 ; 32)$ & 0 & & --- & ---- & 0 & 0 & 0 & 0 & 0 & 1 & 2 \\
\hline$\left(1,1 ; 32^{\prime}\right)$ & $|0|$ & & --- & ---- & 0 & 0 & 0 & 0 & 0 & 1 & -1 \\
\hline$(2,3 ; 1)$ & 0 & & --- & ---- & 3 & 3 & -3 & 0 & 1 & 0 & 0 \\
\hline$(2,3 ; 1)$ & 0 & & --- & ---- & 3 & 3 & -3 & 0 & -1 & 0 & 0 \\
\hline$(2, \overline{3} ; 1)$ & 0 & & --- & ---- & -3 & -3 & 3 & 0 & 1 & 0 & 0 \\
\hline$(2, \overline{3} ; 1)$ & 0 & & --- & ---- & -3 & -3 & 3 & 0 & -1 & 0 & 0 \\
\hline$(2,1 ; 1)$ & 0 & & --- & ---- & -3 & 3 & 3 & 0 & 1 & 0 & 0 \\
\hline$(2,1 ; 1)$ & 0 & & --- & ---- & -3 & 3 & -3 & 0 & 1 & 0 & 0 \\
\hline$(2,1 ; 1)$ & 0 & & --- & ---- & 3 & -3 & 3 & 0 & 1 & 0 & 0 \\
\hline$(2,1 ; 1)$ & 0 & & --- & ---- & 3 & -3 & -3 & 0 & 1 & 0 & 0 \\
\hline$(2,1 ; 1)$ & 0 & & --- & ---- & -3 & 3 & 3 & 0 & -1 & 0 & 0 \\
\hline$(2,1 ; 1)$ & 0 & & --- & ---- & -3 & 3 & -3 & 0 & -1 & 0 & 0 \\
\hline$(2,1 ; 1)$ & 0 & & --- & ---- & 3 & -3 & 3 & 0 & -1 & 0 & 0 \\
\hline$(2,1 ; 1)$ & 0 & & --- & ---- & 3 & -3 & -3 & 0 & -1 & 0 & 0 \\
\hline$(1,3 ; 1)$ & 0 & & --- & ---- & -5 & 1 & 1 & 1 & 1 & 0 & 0 \\
\hline$(1,3 ; 1)$ & 0 & & --- & ---- & 1 & -5 & 1 & 1 & 1 & 0 & 0 \\
\hline$(1,3 ; 1)$ & 0 & & --- & ---- & -5 & 1 & 1 & 1 & -1 & 0 & 0 \\
\hline$(1,3 ; 1)$ & 0 & & --- & ---- & 1 & -5 & 1 & 1 & -1 & 0 & 0 \\
\hline$(1, \overline{3} ; 1)$ & 0 & & --- & ---- & 1 & 1 & 1 & 1 & 1 & 0 & 0 \\
\hline$(1, \overline{3} ; 1)$ & 0 & & --- & ---- & 1 & 1 & -5 & 1 & 1 & 0 & 0 \\
\hline$(1, \overline{3} ; 1)$ & 0 & & --- & ---- & 1 & 1 & 1 & 1 & -1 & 0 & 0 \\
\hline$(1, \overline{3} ; 1)$ & 0 & & --- & ---- & 1 & 1 & -5 & 1 & -1 & 0 & 0 \\
\hline$(1,1 ; 1)$ & 0 & & --- & ---- & 1 & 7 & -5 & 1 & 1 & 0 & 0 \\
\hline$(1,1 ; 1)$ & 0 & & --- & ---- & -5 & -5 & 7 & 1 & 1 & 0 & 0 \\
\hline$(1,1 ; 1)$ & 0 & & --- & ---- & -5 & -5 & 1 & 1 & 1 & 0 & 0 \\
\hline$(1,1 ; 1)$ & 0 & & --- & ---- & 7 & 1 & -5 & 1 & 1 & 0 & 0 \\
\hline$(1,1 ; 1)$ & 0 & & --- & ---- & 1 & 7 & -5 & 1 & -1 & 0 & 0 \\
\hline$(1,1 ; 1)$ & 0 & & --- & ---- & -5 & -5 & 7 & 1 & -1 & 0 & 0 \\
\hline$(1,1 ; 1)$ & 0 & & --- & ---- & -5 & -5 & 1 & 1 & -1 & 0 & 0 \\
\hline$(1,1 ; 1)$ & 0 & & --- & ---- & 7 & 1 & -5 & 1 & -1 & 0 & 0 \\
\hline
\end{tabular}

Table 7. Orbifold massless spectrum after Higgsing. Untwisted sector $k=0$.

where the generators of the five U(1)'s in the first $E_{8}$ are chosen to be

$$
\begin{aligned}
T_{1}^{(1)} & =(0,0,-1,-3,-3,-3,-3,-3), & T_{1}^{(2)}=(0,0,-1,+3,+3,-3,-3,-3), \\
T_{1}^{(3)} & =(0,0,-1,+3,-3,+3,+3,+3), & T_{2}=(0,0,2,0,0,0,0,0), \\
T_{3} & =(+1,-1,0,0,0,0,0,0), &
\end{aligned}
$$




\begin{tabular}{|c|c|c|c|c|c|c|c|c|c|c|c|}
\hline Multiplet & $k$ & $\alpha$ & $\beta$ & $\gamma$ & $q_{1}^{(1)}$ & $q_{1}^{(2)}$ & $q_{1}^{(3)}$ & $q_{2}$ & $q_{3}$ & $\tilde{q}_{2}$ & $\tilde{q}_{4}$ \\
\hline$(2,3 ; 1)$ & 0 & & --- & ---- & -4 & 2 & 2 & -1 & \begin{tabular}{|l|}
0 \\
\end{tabular} & 0 & 0 \\
\hline, $3 ; 1)$ & 0 & & --- & ---- & 2 & -4 & 2 & -1 & 0 & 0 & 0 \\
\hline 1) & 0 & & --- & ---- & 2 & 2 & 2 & -1 & 0 & 0 & 0 \\
\hline ) & 0 & & --- & ---- & 2 & 2 & -4 & -1 & 0 & 0 & 0 \\
\hline, 1 & 0 & & --- & ---- & 2 & 8 & -4 & -1 & 0 & 0 & 0 \\
\hline$(2,1$ & 0 & & --- & ---- & -4 & -4 & 8 & -1 & 0 & 0 & 0 \\
\hline$(2,1$ & 0 & & --- & ---- & -4 & -4 & 2 & -1 & 0 & 0 & 0 \\
\hline$(2,1$ & 0 & & --- & ---- & 8 & 2 & -4 & -1 & 0 & 0 & 0 \\
\hline 2) & 0 & & --- & ---- & 0 & 0 & 0 & 0 & 0 & 2 & 1 \\
\hline & 0 & & --- & ---- & 0 & 0 & 0 & 0 & 0 & 2 & 4 \\
\hline $1 ; 1)$ & 0 & & --- & ---- & 0 & 0 & 0 & 0 & 0 & 2 & -2 \\
\hline $3 ; 1)$ & 0 & & --- & ---- & 2 & 2 & -4 & 2 & 0 & 0 & 0 \\
\hline ;1) & 0 & & --- & ---- & -4 & -4 & 2 & 2 & 0 & 0 & 0 \\
\hline ;1) & 0 & & --- & ---- & -4 & 2 & 2 & 2 & 0 & 0 & 0 \\
\hline 1) & 0 & & --- & ---- & -4 & 2 & -4 & 2 & 0 & 0 & 0 \\
\hline 1) & 0 & & --- & ---- & 2 & -4 & 2 & 2 & 0 & 0 & 0 \\
\hline ;1) & 0 & & --- & ---- & 2 & -4 & -4 & 2 & 0 & 0 & 0 \\
\hline$(2$ & 0 & & --- & ---- & 1 & 1 & 1 & -2 & 1 & 0 & 0 \\
\hline$(2,1$ & 0 & & --- & ---- & 1 & 1 & 1 & -2 & $|-1|$ & 0 & 0 \\
\hline$(1,3 ; 1)$ & 1 & 1 & $1,2,3$ & $1,2,3,4$ & $-13 / 3$ & $5 / 3$ & $5 / 3$ & $-1 / 3$ & 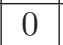 & $2 / 3$ & $1 / 3$ \\
\hline ;1) & 1 & 1 & $, 2,3$ & $1,2,3,4$ & $5 / 3$ & $-13 / 3$ & $5 / 3$ & $-1 / 3$ & 0 & $2 / 3$ & $1 / 3$ \\
\hline ;1) & 1 & 1 & $1,2,3$ & $1,2,3,4$ & $5 / 3$ & $5 / 3$ & $5 / 3$ & $-1 / 3$ & 0 & $2 / 3$ & $1 / 3$ \\
\hline$(1, \overline{3} ; 1)$ & 1 & 1 & $1,2,3$ & $1,2,3,4$ & $5 / 3$ & $5 / 3$ & $-13 / 3$ & $-1 / 3$ & 0 & $2 / 3$ & $1 / 3$ \\
\hline$(1,1 ; 1$ & 1 & 1 & $1,2,3$ & $1,2,3,4$ & $5 / 3$ & $23 / 3$ & $-13 / 3$ & $-1 / 3$ & 0 & $2 / 3$ & $1 / 3$ \\
\hline$(1,1 ; 1)$ & 1 & 1 & $1,2,3$ & $1,2,3,4$ & $-13 / 3$ & $-13 / 3$ & $23 / 3$ & $-1 / 3$ & 0 & $2 / 3$ & $1 / 3$ \\
\hline (1 & 1 & 1 & $1,2,3$ & $1,2,3,4$ & $-13 / 3$ & $-13 / 3$ & $5 / 3$ & $-1 / 3$ & 0 & $2 / 3$ & $1 / 3$ \\
\hline$(1,1 ; 1)$ & 1 & 1 & $1,2,3$ & $1,2,3,4$ & $23 / 3$ & $5 / 3$ & $-13 / 3$ & $-1 / 3$ & 0 & $2 / 3$ & $1 / 3$ \\
\hline$(2,1 ; 1)$ & 1 & 1 & $1,2,3$ & $1,2,3,4$ & $-1 / 3$ & $-1 / 3$ & $-1 / 3$ & $2 / 3$ & 0 & $2 / 3$ & $1 / 3$ \\
\hline$(2,1 ; 1)$ & 1 & 1 & $1,2,3$ & $1,2,3,4$ & $-1 / 3$ & $-1 / 3$ & $-1 / 3$ & $2 / 3$ & 0 & $2 / 3$ & $1 / 3$ \\
\hline$(1,1 ; 1)$ & 1 & 1 & $1,2,3$ & $1,2,3,4$ & $2 / 3$ & $2 / 3$ & $2 / 3$ & $-4 / 3$ & 1 & $2 / 3$ & $1 / 3$ \\
\hline$(1,1 ; 1)$ & 1 & 1 & $1,2,3$ & $1,2,3,4$ & $2 / 3$ & $2 / 3$ & $2 / 3$ & $-4 / 3$ & -1 & $2 / 3$ & $1 / 3$ \\
\hline$(1,1 ; 1)$ & 2 & 1 & 2,3 & - & $-2 / 3$ & $-2 / 3$ & $-2 / 3$ & $4 / 3$ & 0 & $4 / 3$ & $2 / 3$ \\
\hline$(2,1 ; 1)$ & 2 & 1 & 2,3 & - & $1 / 3$ & $1 / 3$ & $1 / 3$ & $-2 / 3$ & 1 & $4 / 3$ & $2 / 3$ \\
\hline$(2,1 ; 1)$ & 2 & 1 & $1,2,3$ & - & $1 / 3$ & $1 / 3$ & $1 / 3$ & $-2 / 3$ & -1 & $4 / 3$ & $2 / 3$ \\
\hline$(1,1 ; 1)$ & 2 & $3+5$ & 2,3 & - & $-2 / 3$ & $-2 / 3$ & $-2 / 3$ & $4 / 3$ & 0 & $4 / 3$ & $2 / 3$ \\
\hline$(2,1 ; 1)$ & 2 & $3+5$ & $1,2,3$ & - & $1 / 3$ & $1 / 3$ & $1 / 3$ & $-2 / 3$ & 1 & $4 / 3$ & $2 / 3$ \\
\hline$(2,1 ; 1)$ & 2 & $3+5$ & $1,2,3$ & - & $1 / 3$ & $1 / 3$ & $1 / 3$ & $-2 / 3$ & -1 & $4 / 3$ & $2 / 3$ \\
\hline$(1,1 ; 1)$ & 2 & $3-5$ & $1,2,3$ & - & $-2 / 3$ & $-2 / 3$ & $-2 / 3$ & $4 / 3$ & 0 & $4 / 3$ & $2 / 3$ \\
\hline$(1,3 ; 1)$ & 2 & $3-5$ & $1,2,3$ & - & $10 / 3$ & $10 / 3$ & $-8 / 3$ & $-2 / 3$ & 0 & $4 / 3$ & $2 / 3$ \\
\hline$(1, \overline{3} ; 1)$ & 2 & $3-5$ & $1,2,3$ & - & $-8 / 3$ & $-8 / 3$ & $10 / 3$ & $-2 / 3$ & 0 & $4 / 3$ & $2 / 3$ \\
\hline$(1,1 ; 1)$ & 2 & $3-5$ & $1,2,3$ & - & $-8 / 3$ & $10 / 3$ & $10 / 3$ & $-2 / 3$ & 0 & $4 / 3$ & $2 / 3$ \\
\hline$(1,1 ; 1)$ & 2 & $3-5$ & $1,2,3$ & - & $-8 / 3$ & $10 / 3$ & $-8 / 3$ & $-2 / 3$ & 0 & $4 / 3$ & $2 / 3$ \\
\hline
\end{tabular}

Table 8. Orbifold massless spectrum after higgsing. Untwisted sector $k=0$ continued; twisted sectors $k=1$ and $k=2$. 


\begin{tabular}{|c|c|c|c|c|c|c|c|c|c|c|c|}
\hline Multiplet & $k$ & $\alpha$ & $\beta$ & $\gamma$ & $q_{1}^{(1)}$ & $q_{1}^{(2)}$ & $q_{1}^{(3)}$ & $q_{2}$ & $q_{3}$ & $\tilde{q}_{2}$ & $\tilde{q}_{4}$ \\
\hline$(1,1 ; 1)$ & 2 & $3-5$ & $1,2,3$ & - & $10 / 3$ & $-8 / 3$ & $10 / 3$ & $-2 / 3$ & 0 & $4 / 3$ & $2 / 3$ \\
\hline $1 ; 1)$ & 2 & $3-5$ & $1,2,3$ & - & $10 / 3$ & $-8 / 3$ & $-8 / 3$ & $-2 / 3$ & 0 & $4 / 3$ & $2 / 3$ \\
\hline & & $3-5$ & 2,3 & - & $-2 / 3$ & $-2 / 3$ & $-2 / 3$ & $4 / 3$ & 0 & $-2 / 3$ & $-1 / 3$ \\
\hline & & $3-5$ & 2,3 & - & $-2 / 3$ & $-2 / 3$ & $-2 / 3$ & $4 / 3$ & 0 & $-2 / 3$ & $8 / 3$ \\
\hline 1,1 & 2 & $3-5$ & $1,2,3$ & - & $-2 / 3$ & $-2 / 3$ & $-2 / 3$ & $4 / 3$ & 0 & $-2 / 3$ & $-10 /$ \\
\hline & & 1 & - & 2,3, & 0 & 0 & 0 & 0 & 1 & ( & 1 \\
\hline & 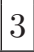 & 1 & - & 2,3 & c & 0 & & ( & -1 & & 1 \\
\hline & 20 & $+4+6$ & - & 2,3 & c & c & 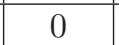 & 0 & 1 & 2 & 1 \\
\hline$(1$, & o & $2+4+6$ & - & 2,3 & 0 & 0 & 0 & 0 & -1 & 2 & 1 \\
\hline$(1,1 ; 1)$ & 3 & $2+\nu 4+\nu^{2} 6$ & - & $1,2,3,4$ & 0 & 0 & 0 & 0 & 1 & -2 & -1 \\
\hline$(1,1$ & 3 & $2+\nu 4+\nu^{2} 6$ & - & $1,2,3,4$ & 0 & 0 & 0 & 0 & -1 & -2 & -1 \\
\hline & & $2+\nu^{2} 4+\nu^{4} 6$ & - & & 0 & c & 0 & 0 & 1 & 0 & 0 \\
\hline & & $2+\nu^{2} 4+\nu^{4} 6$ & - & & 0 & 0 & 0 & 0 & -1 & 0 & 0 \\
\hline & 3 & $2+\nu^{2} 4+\nu^{4} 6$ & - & 2,3 & 0 & c & 0 & 0 & 1 & U & 3 \\
\hline & 3 & $2+\nu^{2} 4+\nu^{4} 6$ & - & 2,3 & 0 & 0 & 0 & 0 & -1 & 0 & 3 \\
\hline & 3 & $2+\nu^{2} 4+\nu^{4} 6$ & - & $1,2,3,4$ & 0 & 0 & 0 & 0 & 1 & 0 & -3 \\
\hline$(1$, & 3 & $2+\nu^{2} 4+\nu^{4} 6$ & - & $1,2,3,4$ & 0 & 0 & 0 & 0 & -1 & 0 & -3 \\
\hline & . & 1 & $1,2,3$ & - & $2 / 3$ & $2 / 3$ & $2 / 3$ & $-4 / 3$ & 0 & $-4 / 3$ & $-2 / 3$ \\
\hline & & 1 & $1,2,3$ & - & $8 / 3$ & 8 & $-10 / 3$ & $2 / 3$ & 0 & $-4 / 3$ & $-2 / 3$ \\
\hline & & 1 &, 3 & - & $-10 / 3$ & $-10 / 3$ & $8 / 3$ & $2 / 3$ & 0 & $-4 / 3$ & $-2 / 3$ \\
\hline & 4 & 1 & 2,3 & - & $-10 / 3$ & $8 / 3$ & $8 / 3$ & $2 / 3$ & 0 & $-4 / 3$ & $-2 / 3$ \\
\hline & 4 & 1 & 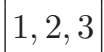 & - & $-10 / 3$ & $8 / 3$ & $-10 / 3$ & $2 / 3$ & 0 & $-4 / 3$ & $-2 / 3$ \\
\hline & 4 & 1 & 3 & - & $8 / 3$ & $-10 / 3$ & $8 / 3$ & $2 / 3$ & 0 & $-4 / 3$ & $-2 / 3$ \\
\hline & 4 & 1 & 2,3 & - & $8 / 3$ & $-10 / 3$ & $-10 / 3$ & $2 / 3$ & 0 & $-4 / 3$ & $-2 / 3$ \\
\hline & & 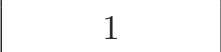 & 2 & - & $2 / 3$ & $2 / 3$ & $2 / 3$ & $-4 / 3$ & 0 & $2 / 3$ & $1 / 3$ \\
\hline & 4 & 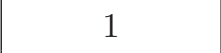 &, 3 & - & $2 / 3$ & 2 & $2 / 3$ & $-4 / 3$ & 0 & $2 / 3$ & $10 / 3$ \\
\hline & 4 & 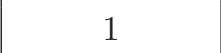 & $1,2,3$ & - & $2 / 3$ & 2 & $2 / 3$ & $-4 / 3$ & 0 & $2 / 3$ & $-8 / 3$ \\
\hline & & & 2,3 & - & $2 / 3$ & $2 / 3$ & $2 / 3$ & $-4 / 3$ & 0 & $-4 / 3$ & $-2 / 3$ \\
\hline & 4 & & D & - & $8 / 3$ & $8 / 3$ & $-10 / 3$ & $2 / 3$ & 0 & $-4 / 3$ & $-2 / 3$ \\
\hline & 4 & & 3 & - & $-10 / 3$ & $-10 / 3$ & $8 / 3$ & $2 / 3$ & 0 & $-4 / 3$ & $-2 / 3$ \\
\hline & 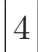 & & & - & $-10 / 3$ & $8 / 3$ & $8 / 3$ & $2 / 3$ & 0 & $-4 / 3$ & $-2 / 3$ \\
\hline & . & & & - & $-10 / 3$ & $8 / 3$ & $-10 / 3$ & $2 / 3$ & 0 & $-4 / 3$ & $-2 / 3$ \\
\hline & 4 & & & - & $8 / 3$ & $-10 / 3$ & $8 / 3$ & $2 / 3$ & 0 & $-4 / 3$ & $-2 / 3$ \\
\hline & 4 & &, 3 & - & $8 / 3$ & $-10 / 3$ & $-10 / 3$ & $2 / 3$ & 0 & $-4 / 3$ & $-2 / 3$ \\
\hline & & & & - & $2 /:$ & $2 / 3$ & $2 / 3$ & $-4 / 3$ & 0 & $2 / 3$ & $1 / 3$ \\
\hline & 4 & & & - & $2 / 3$ & $2 / 3$ & $2 / 3$ & $-4 / 3$ & 0 & $2 / 3$ & $10 / 3$ \\
\hline & 4 & & 3 & - & $2 / 3$ & $2 / 3$ & $2 / 3$ & $-4 / 3$ & 0 & $2 / 3$ & $-8 / 3$ \\
\hline & 4 & & 3 & - & $2 / 3$ & $2 / 3$ & $2 / 3$ & $-4 / 3$ & 0 & $-4 / 3$ & $-2 / 3$ \\
\hline & 4 & & $1,2,0$ & - & $-1 / 3$ & $-1 / 3$ & $-1 / 3$ & $2 / 3$ & 1 & $-4 / 3$ & $-2 / 3$ \\
\hline $2,1,1)$ & 4 & $3-5$ & $1,2,3$ & - & $-1 / 3$ & $-1 / 3$ & $-1 / 3$ & $2 / 3$ & -1 & $-4 / 3$ & $-2 / 3$ \\
\hline
\end{tabular}

Table 9. Orbifold massless spectrum after Higgsing. Twisted sectors $k=2$ (continued), $k=3$ and $k=4$. 


\begin{tabular}{|c|c|c|c|c|c|c|c|c|c|c|c|c|c|}
\hline State & $q_{1}^{(1)}$ & $q_{1}^{(2)}$ & $q_{1}^{(3)}$ & $q_{2}$ & $q_{3}$ & $n$ & State & $q_{1}^{(1)}$ & $q_{1}^{(2)}$ & $q_{1}^{(3)}$ & $q_{2}$ & $q_{3}$ & $n$ \\
\hline $\mathbf{3}, \mathbf{1})$ & -5 & 1 & 1 & 1 & 1 & -1 & $(\mathbf{3}, \mathbf{2})$ & -2 & -2 & -2 & 1 & 0 & 1 \\
$(\mathbf{3}, \mathbf{1})$ & 1 & -5 & 1 & 1 & 1 & -1 & $(\mathbf{3}, \mathbf{2})$ & -2 & -2 & 4 & 1 & 0 & 1 \\
$(\overline{\mathbf{3}}, \mathbf{1})$ & 1 & 1 & -5 & 1 & 1 & -1 & $(\overline{\mathbf{3}}, \mathbf{2})$ & -2 & 4 & -2 & 1 & 0 & 1 \\
$(\overline{\mathbf{3}}, \mathbf{1})$ & 1 & 1 & 1 & 1 & 1 & -1 & $(\overline{\mathbf{3}}, \mathbf{2})$ & 4 & -2 & -2 & 1 & 0 & 1 \\
$(\mathbf{1}, \mathbf{1})$ & -5 & -5 & 7 & 1 & 1 & 5 & $(\mathbf{1}, \mathbf{2})$ & -8 & -2 & 4 & 1 & 0 & -5 \\
$(\mathbf{1}, \mathbf{1})$ & -5 & -5 & 7 & 1 & 1 & 5 & $(\mathbf{1}, \mathbf{2})$ & -2 & -8 & 4 & 1 & 0 & -5 \\
$(\mathbf{1}, \mathbf{1})$ & 7 & 1 & -5 & 1 & 1 & 5 & $(\mathbf{1}, \mathbf{2})$ & 4 & 4 & -8 & 1 & 0 & -5 \\
$(\mathbf{1}, \mathbf{1})$ & 1 & 7 & -5 & 1 & 1 & 5 & $(\mathbf{1}, \mathbf{2})$ & 4 & 4 & -2 & 1 & 0 & -5 \\
$(\mathbf{3}, \mathbf{1})$ & -5 & 1 & 1 & 1 & -1 & -1 & $(\mathbf{1}, \mathbf{2})$ & -1 & -1 & -1 & 2 & 1 & 4 \\
$(\mathbf{3}, \mathbf{1})$ & 1 & -5 & 1 & 1 & -1 & -1 & $(\mathbf{1}, \mathbf{2})$ & -1 & -1 & -1 & 2 & -1 & 4 \\
$(\overline{\mathbf{3}}, \mathbf{1})$ & 1 & 1 & -5 & 1 & -1 & -1 & $(\mathbf{3}, \mathbf{1})$ & 2 & 2 & -4 & 2 & 0 & 2 \\
$(\overline{\mathbf{3}}, \mathbf{1})$ & 1 & 1 & 1 & 1 & -1 & -1 & $(\overline{\mathbf{3}}, \mathbf{1})$ & -4 & -4 & 2 & 2 & 0 & 2 \\
$(\mathbf{1}, \mathbf{1})$ & -5 & -5 & 7 & 1 & -1 & 5 & $(\mathbf{1}, \mathbf{1})$ & 2 & -4 & -4 & 2 & 0 & 2 \\
$(\mathbf{1}, \mathbf{1})$ & -5 & -5 & 7 & 1 & -1 & 5 & $(\mathbf{1}, \mathbf{1})$ & -4 & 2 & -4 & 2 & 0 & 2 \\
$(\mathbf{1}, \mathbf{1})$ & 7 & 1 & -5 & 1 & -1 & 5 & $(\mathbf{1}, \mathbf{1})$ & -4 & 2 & 2 & 2 & 0 & 2 \\
$(\mathbf{1}, \mathbf{1})$ & 1 & 7 & -5 & 1 & -1 & 5 & $(\mathbf{1}, \mathbf{1})$ & 2 & -4 & 2 & 2 & 0 & 2 \\
$(\mathbf{3}, \mathbf{1})$ & 0 & 6 & -6 & 0 & 0 & 0 & $(\mathbf{1}, \mathbf{1})$ & 0 & 0 & 0 & 0 & 2 & 4 \\
$(\mathbf{3}, \mathbf{1})$ & 0 & 6 & 0 & 0 & 0 & 0 & $(\mathbf{3}, \mathbf{2})$ & 3 & 3 & -3 & 0 & -1 & 0 \\
$(\overline{\mathbf{3}}, \mathbf{1})$ & -6 & 0 & 6 & 0 & 0 & 0 & $(\overline{\mathbf{3}}, \mathbf{2})$ & -3 & -3 & 3 & 0 & -1 & 0 \\
$(\overline{\mathbf{3}}, \mathbf{1})$ & -6 & 0 & 0 & 0 & 0 & 0 & $(\mathbf{1}, \mathbf{2})$ & 3 & -3 & -3 & 0 & -1 & 0 \\
$(\mathbf{1}, \mathbf{1})$ & -6 & 6 & 0 & 0 & 0 & 0 & $(\mathbf{1}, \mathbf{2})$ & 3 & -3 & 3 & 0 & -1 & 0 \\
$(\mathbf{1}, \mathbf{1})$ & 0 & 0 & 6 & 0 & 0 & 0 & $(\mathbf{1}, \mathbf{2})$ & -3 & 3 & 3 & 0 & -1 & 0 \\
$(\mathbf{3}, \mathbf{1})$ & -6 & -6 & 6 & 0 & 0 & 0 & $(\mathbf{1}, \mathbf{2})$ & -3 & 3 & -3 & 0 & -1 & 0 \\
\hline
\end{tabular}

Table 10. Representation $\mathbf{R}^{(1)}$ in the decomposition (C.4) of the first $E_{8}$.

and the generators of the two U(1)'s in the second $E_{8}$ are

$$
\tilde{T}_{2}=(2,0,0,0,0,0,0,0), \quad \tilde{T}_{4}=(1,3,0,0,0,0,0,0) .
$$

We now list the spectrum which arises from the decomposition of the adjoint representation of the two $E_{8}$ factors,

$$
\begin{aligned}
& \mathbf{2 4 8}^{(1)}=(\mathbf{1}, \mathbf{3}) \oplus(\mathbf{8}, \mathbf{1}) \oplus(\mathbf{1}, \mathbf{1})^{5} \oplus\left(\mathbf{R}^{(\mathbf{1})} \oplus \overline{\mathbf{R}}^{(\mathbf{1})}\right), \\
& \mathbf{2 4 8}^{(2)}=\mathbf{6 6} \oplus \mathbf{1} \oplus \mathbf{1} \oplus\left(\mathbf{R}^{(\mathbf{2})} \oplus \overline{\mathbf{R}}^{(\mathbf{2})}\right) .
\end{aligned}
$$




\begin{tabular}{|r|c|c|c|r|c|c|c|r|c|c|c|}
\hline State & $\tilde{q}_{2}$ & $\tilde{q}_{4}$ & $n$ & State & $\tilde{q}_{2}$ & $\tilde{q}_{4}$ & $n$ & State & $\tilde{q}_{2}$ & $\tilde{q}_{4}$ & $n$ \\
\hline $\mathbf{3 2}$ & 1 & 2 & -1 & $\mathbf{1 2}$ & 2 & 1 & -1 & $\mathbf{1}$ & 2 & 4 & -10 \\
$\mathbf{3 2}^{\prime}$ & 1 & -1 & -1 & $\mathbf{1 2}$ & 0 & 3 & 3 & $\mathbf{1}$ & 2 & -2 & 8 \\
\hline
\end{tabular}

Table 11. Representation $\mathbf{R}^{(2)}$ in the decomposition (C.5) of the first $E_{8}$.

Each state is given together with its charges under the U(1) generators (C.2) and (C.3). Moreover, the chiral index is given for each state. It is obtained by applying the number operator (3.59) to the state. Since the fluxes $f_{I}$ are linear combinations of the above U(1) generators, the chiral index will be give in terms of the charges. For the case under study, the chiral multiplicity of a state of charges $\left(q_{1}^{(1)}, q_{1}^{(2)}, q_{1}^{(3)}, q_{2}, q_{3}, \tilde{q}_{2}, \tilde{q}_{4}\right)$ is

$$
\begin{aligned}
n=\frac{1}{108}[ & 52 q_{2}^{3}+3 q_{2}\left(\left(q_{1}^{(1)}\right)^{2}+\left(q_{1}^{(2)}\right)^{2}+\left(q_{1}^{(3)}\right)^{2}+\left(q_{1}^{(1)}+q_{1}^{(2)}+q_{1}^{(3)}+2 q_{2}\right)^{2}\right) \\
& +2\left(\left(q_{1}^{(1)}\right)^{3}+\left(q_{1}^{(2)}\right)^{3}+\left(q_{1}^{(3)}\right)^{3}-\left(q_{1}^{(1)}+q_{1}^{(2)}+q_{1}^{(3)}+2 q_{2}\right)^{3}\right)+108 q_{2} q_{3}^{2} \\
& \left.+72 q_{3}^{3}-36\left(3 q_{2}+2 q_{3}\right)\right]-\frac{1}{3}\left[5 \tilde{q}_{2}+2 \tilde{q}_{2}^{3}+3 \tilde{q}_{4}-3 \tilde{q}_{2}^{2} \tilde{q}_{4}\right] .
\end{aligned}
$$

In tables 10 (11) we display the states which arise from the decomposition of the adjoint representation of the first (second) $E_{8}$. For the first $E_{8}$ all of them have $\tilde{q}_{2}=0$ and $\tilde{q}_{4}=0$, while for the second $E_{8}$ all of them have $q_{1}^{i}=0$ and $q_{2}=q_{3}=0$. The adjoint decomposes into the sum of the adjoint representations of the unbroken group, plus the sum of the reducible representation $\mathbf{R}$ and its complex conjugate $\overline{\mathbf{R}}$. In both tables we only list the irreducible representation coming from $\mathbf{R}$.

Open Access. This article is distributed under the terms of the Creative Commons Attribution License which permits any use, distribution and reproduction in any medium, provided the original author(s) and source are credited.

\section{References}

[1] L.J. Dixon, J.A. Harvey, C. Vafa and E. Witten, Strings on orbifolds, Nucl. Phys. B 261 (1985) 678 [inSPIRE].

[2] L.J. Dixon, J.A. Harvey, C. Vafa and E. Witten, Strings on orbifolds. 2, Nucl. Phys. B 274 (1986) 285 [inSPIRE].

[3] Y. Katsuki et al., $Z_{N}$ orbifold models, Nucl. Phys. B 341 (1990) 611 [INSPIRE].

[4] T. Kobayashi, S. Raby and R.-J. Zhang, Searching for realistic 4d string models with a Pati-Salam symmetry: orbifold grand unified theories from heterotic string compactification on a $Z_{6}$ orbifold, Nucl. Phys. B 704 (2005) 3 [hep-ph/0409098] [INSPIRE].

[5] W. Buchmüller, K. Hamaguchi, O. Lebedev and M. Ratz, Supersymmetric standard model from the heterotic string, Phys. Rev. Lett. 96 (2006) 121602 [hep-ph/0511035] [INSPIRE].

[6] W. Buchmüller, K. Hamaguchi, O. Lebedev and M. Ratz, Supersymmetric standard model from the heterotic string (II), Nucl. Phys. B 785 (2007) 149 [hep-th/0606187] [INSPIRE]. 
[7] O. Lebedev et al., A mini-landscape of exact MSSM spectra in heterotic orbifolds, Phys. Lett. B 645 (2007) 88 [hep-th/0611095] [INSPIRE].

[8] O. Lebedev, H.P. Nilles, S. Ramos-Sanchez, M. Ratz and P.K. Vaudrevange, Heterotic mini-landscape. (II). Completing the search for MSSM vacua in a $Z_{6}$ orbifold, Phys. Lett. B 668 (2008) 331 [arXiv:0807.4384] [INSPIRE].

[9] M. Blaszczyk et al., A $Z_{2} \times Z_{2}$ standard model, Phys. Lett. B 683 (2010) 340 [arXiv:0911.4905] [INSPIRE].

[10] G. Honecker and M. Trapletti, Merging heterotic orbifolds and K3 compactifications with line bundles, JHEP 01 (2007) 051 [hep-th/0612030] [INSPIRE].

[11] S. Groot Nibbelink, M. Trapletti and M. Walter, Resolutions of $C^{n} / Z_{N}$ orbifolds, their U(1) bundles and applications to string model building, JHEP 03 (2007) 035 [hep-th/0701227] [INSPIRE].

[12] S. Nibbelink Groot, T.-W. Ha and M. Trapletti, Toric resolutions of heterotic orbifolds, Phys. Rev. D 77 (2008) 026002 [arXiv:0707.1597] [InSPIRE].

[13] S. Nibbelink Groot, D. Klevers, F. Ploger, M. Trapletti and P.K. Vaudrevange, Compact heterotic orbifolds in blow-up, JHEP 04 (2008) 060 [arXiv:0802.2809] [INSPIRE].

[14] S. Nibbelink Groot, J. Held, F. Ruehle, M. Trapletti and P.K. Vaudrevange, Heterotic $Z_{6-I I}$ MSSM orbifolds in blowup, JHEP 03 (2009) 005 [arXiv:0901.3059] [INSPIRE].

[15] M. Blaszczyk, S. Nibbelink Groot, F. Ruehle, M. Trapletti and P.K. Vaudrevange, Heterotic MSSM on a resolved orbifold, JHEP 09 (2010) 065 [arXiv: 1007.0203] [INSPIRE].

[16] M. Blaszczyk, N.G. Cabo Bizet, H.P. Nilles and F. Ruhle, A perfect match of MSSM-like orbifold and resolution models via anomalies, JHEP 10 (2011) 117 [arXiv:1108.0667] [INSPIRE].

[17] D. Lüst, S. Reffert, E. Scheidegger and S. Stieberger, Resolved toroidal orbifolds and their orientifolds, Adv. Theor. Math. Phys. 12 (2008) 67 [hep-th/0609014] [InSPIRE].

[18] S. Nibbelink Groot, Heterotic orbifold resolutions as $(2,0)$ gauged linear $\sigma$-models, Fortsch. Phys. 59 (2011) 454 [arXiv: 1012.3350] [INSPIRE].

[19] M. Blaszczyk, S. Nibbelink Groot and F. Ruehle, Green-Schwarz mechanism in heterotic $(2,0)$ gauged linear $\sigma$-models: torsion and NS5 branes, JHEP 08 (2011) 083 [arXiv:1107.0320] [INSPIRE].

[20] M. Blaszczyk, S. Groot Nibbelink and F. Ruehle, Gauged linear $\sigma$-models for toroidal orbifold resolutions, JHEP 05 (2012) 053 [arXiv:1111.5852] [INSPIRE].

[21] W. Buchmüller, C. Lüdeling and J. Schmidt, Local SU(5) unification from the heterotic string, JHEP 09 (2007) 113 [arXiv:0707.1651] [INSPIRE].

[22] W. Buchmüller and J. Schmidt, Higgs versus matter in the heterotic landscape, Nucl. Phys. B 807 (2009) 265 [arXiv:0807.1046] [INSPIRE].

[23] C. Voisin, Miroirs et involutions sur les surfaces K3 (in French), in Journées de Géométrie Algébrique d'Orsay, France July 1992.

[24] C. Borcea, K3 surfaces with involution and mirror pairs of Calabi-Yau manifolds, in Mirror symmetry II, B. Greene and S.T. Yau eds., AMS/IP Stud. Adv. Math. 1, American Mathematical Society, Providence U.S.A. (1997), pg. 717 [INSPIRE]. 
[25] G. Honecker, Massive U(1)s and heterotic five-branes on K3, Nucl. Phys. B 748 (2006) 126 [hep-th/0602101] [INSPIRE].

[26] S. Nibbelink Groot, F.P. Correia and M. Trapletti, Non-Abelian bundles on heterotic non-compact K3 orbifold blowups, JHEP 11 (2008) 044 [arXiv:0809.4430] [INSPIRE].

[27] J. Louis, M. Schasny and R. Valandro, $6 D$ effective action of heterotic compactification on $K 3$ with nontrivial gauge bundles, JHEP 04 (2012) 028 [arXiv:1112.5106] [INSPIRE].

[28] P.S. Aspinwall, K3 surfaces and string duality, hep-th/9611137 [INSPIRE].

[29] K. Dasgupta and S. Mukhi, F-theory at constant coupling, Phys. Lett. B 385 (1996) 125 [hep-th/9606044] [INSPIRE].

[30] L.J. Hall, H. Murayama and Y. Nomura, Wilson lines and symmetry breaking on orbifolds, Nucl. Phys. B 645 (2002) 85 [hep-th/0107245] [INSPIRE].

[31] F. Hirzebruch, Topological methods in algebraic geometry, $3^{\text {rd }}$ edition, Grundlehren Math. Wiss. 131, Springer-Verlag, New York U.S.A. (1978).

[32] J. Schmidt, Local grand unification in the heterotic landscape, Fortsch. Phys. 58 (2010) 3 [arXiv: 0906.5501] [INSPIRE].

[33] H.P. Nilles, S. Ramos-Sanchez, P.K. Vaudrevange and A. Wingerter, The orbifolder: a tool to study the low energy effective theory of heterotic orbifolds,

Comput. Phys. Commun. 183 (2012) 1363 [arXiv:1110.5229] [INSPIRE].

[34] F. Buccella, J. Derendinger, S. Ferrara and C.A. Savoy, Patterns of symmetry breaking in supersymmetric gauge theories, Phys. Lett. B 115 (1982) 375 [InSPIRE].

[35] J. Casas, E. Katehou and C. Muñoz, U(1) charges in orbifolds: anomaly cancellation and phenomenological consequences, Nucl. Phys. B 317 (1989) 171 [INSPIRE].

[36] G. Cleaver, M. Cvetič, J.R. Espinosa, L.L. Everett and P. Langacker, Classification of flat directions in perturbative heterotic superstring vacua with anomalous $\mathrm{U}(1)$,

Nucl. Phys. B 525 (1998) 3 [hep-th/9711178] [INSPIRE].

[37] G. Cleaver, M. Cvetič, J.R. Espinosa, L.L. Everett and P. Langacker, Flat directions in three generation free fermionic string models, Nucl. Phys. B 545 (1999) 47 [hep-th/9805133] [INSPIRE].

[38] A.P. Braun, R. Ebert, A. Hebecker and R. Valandro, Weierstrass meets Enriques, JHEP 02 (2010) 077 [arXiv:0907.2691] [INSPIRE].

[39] V.V. Nikulin, On factor groups of the automorphism group of hyperbolic forms modulo subgroups generated by 2-reections, Sov. Math. Dokl. 20 (1979) 1156.

[40] V.V. Nikulin, Quotient-groups of groups of automorphisms of hyperbolic forms by subgroups generated by 2-reections, Algebro-geometric applications, J. Soviet Math. 22 (1983) 1401.

[41] V.V. Nikulin, Discrete reection groups in Lobachevsky spaces and algebraic surfaces, in Proceedings of the International Congress of Mathematicians, Berkeley U.S.A. 1986, volume 1, American Mathematical Society, Providence U.S.A. (1988).

[42] V.V. Nikulin, Discrete reection groups in Lobachevsky spaces and algebraic surfaces, in Proceedings of the International Congress of Mathematicians, Berkeley U.S.A. 1986, volume 2, American Mathematical Society, Providence U.S.A. (1988).

[43] D.R. Morrison and C. Vafa, Compactifications of F-theory on Calabi-Yau threefolds. 2, Nucl. Phys. B 476 (1996) 437 [hep-th/9603161] [INSPIRE]. 
[44] R. Blumenhagen, G. Honecker and T. Weigand, Loop-corrected compactifications of the heterotic string with line bundles, JHEP 06 (2005) 020 [hep-th/0504232] [INSPIRE].

[45] J. Distler and B.R. Greene, Aspects of $(2,0)$ string compactifications, Nucl. Phys. B 304 (1988) 1 [INSPIRE].

[46] E. Witten, Global anomalies in string theory, Print-85-0620, Princeton U.S.A. (1985) [INSPIRE].

[47] D. Freed, Determinants, torsion, and strings, Commun. Math. Phys. 107 (1986) 483 [INSPIRE].

[48] T. Weigand, Compactifications of the heterotic string with unitary bundles, Fortsch. Phys. 54 (2006) 963 [INSPIRE].

[49] M.B. Green, J.H. Schwarz and E. Witten, Superstring theory. Volume 2: loop amplitudes, anomalies and phenomenology, Cambridge Monographs On Mathematical Physics, Cambridge University Press, Cambridge U.K. (1987) [INSPIRE]. 